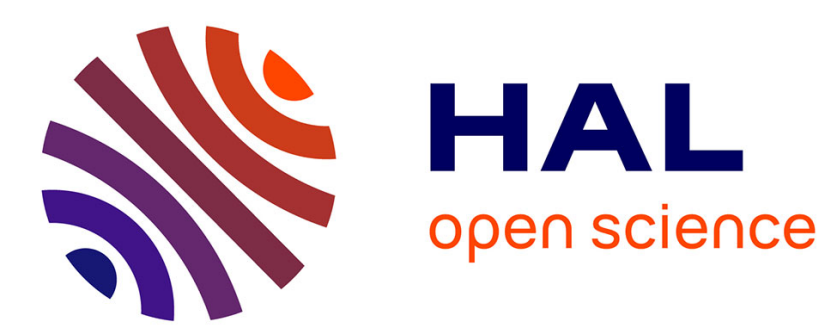

\title{
Moment-of-fluid analytic reconstruction on 3D rectangular hexahedrons
}

Thomas Milcent, Antoine Lemoine

\section{To cite this version:}

Thomas Milcent, Antoine Lemoine. Moment-of-fluid analytic reconstruction on 3D rectangular hexahedrons. Journal of Computational Physics, 2020, 409, pp.109346. 10.1016/j.jcp.2020.109346 . hal03388524

\section{HAL Id: hal-03388524 \\ https://hal.science/hal-03388524}

Submitted on 20 Oct 2021

HAL is a multi-disciplinary open access archive for the deposit and dissemination of scientific research documents, whether they are published or not. The documents may come from teaching and research institutions in France or abroad, or from public or private research centers.
L'archive ouverte pluridisciplinaire HAL, est destinée au dépôt et à la diffusion de documents scientifiques de niveau recherche, publiés ou non, émanant des établissements d'enseignement et de recherche français ou étrangers, des laboratoires publics ou privés. 


\title{
Moment-of-Fluid Analytic Reconstruction on 3D Rectangular Hexahedrons
}

\author{
Thomas Milcent $^{\mathrm{a}}$, Antoine Lemoine ${ }^{\mathrm{b}}$ \\ ${ }^{a}$ Arts et Métiers ParisTech, I2M, UMR 5295, F-33400 Talence, France. \\ ${ }^{b}$ Bordeaux INP, I2M, UMR 5295, F-33400 Talence, France.
}

\begin{abstract}
The moment-of-fluid method (MOF) is a second-order accurate interface reconstruction method which can be seen as an extension of the volume-of-fluid method with piecewise linear interface construction (VOF-PLIC). MOF involves a computationally intensive minimization problem that needs to be solved on every cell containing several materials. We propose a new fast and robust reconstruction algorithm to tackle this problem on rectangular hexahedral cells. Our approach uses explicit analytic formulas of the objective function that does not use any geometric computations such as half-space-polyhedron intersections. The numerical results show that the proposed method is more robust and more than 200 times faster than the original approach. Additionally, we propose a faster reconstruction algorithm on convex polyhedral cells. All the methods presented in this article have been implemented and verified on the open-source code Notus.
\end{abstract}

Keywords: Moment-of-Fluid, interface reconstruction, material tracking, PLIC, centroid locus

\section{Introduction}

Common engineering problems involve several materials interacting with each other. The numerical simulations of these phenomena require the tracking of the location of the materials over time. Across the interface between two materials, some physical phenomena must be described such as the heat or mass transfer. Any numerical errors on the location of the interface have an impact on the physics of the whole problem. As a result, the numerical simulations require accurate tracking methods. In this article we only consider numerical methods designed for the Eulerian framework where the velocity field is defined on the whole domain and where the motion of the materials is independent of the underlying mesh.

A lot numerical strategies have been developed to track the materials in this context such as the level-set method [1], the front-tracking method [2], and the volume-of-fluid method with piecewise linear interface construction (VOF-PLIC). For the latter, any cell containing two materials is partitioned by a linear interface such that the volume of each part contains exactly the same volume as the real location of the material. The most common application of the VOF-PLIC method is the advection of the materials which is composed of two steps. In the first step, the geometry of the partition is advected with a Lagrangian method, and then the volume of each material is computed from the intersection of the geometry with the underlying mesh. In the second step, named reconstruction, a new partition is computed using the volume in each cells and their neighborhood.

Recently the moment-of-fluid method (MOF) [3-22] was introduced as a second-order accurate extension of the VOF-PLIC method for multi-material reconstruction. Besides the volume of the materials, MOF involves the centroids of each material which allows to reconstruct the partition with only the information contained in the cell. Furthermore, this method offers a straightforward way to represent $n \geq 2$ materials in the same cell [5,6]. The improved accuracy of this method is at the expense of the time to partition the cell which involves a computationally intensive minimization problem. In this article, we present a fast and

Email addresses: thomas.milcent@u-bordeaux.fr (Thomas Milcent), antoine.lemoine@bordeaux-inp.fr (Antoine Lemoine) 
robust reconstruction strategy to solve this minimization problem on rectangular hexahedrons. Note that we only address the problem of the reconstruction, the advection can be done, for instance, using a Lagrangian remapping method [4] or a directional splitting method [12].

The currently proposed method falls under the continuity of the improvements made to MOF during the past decade. MOF was originally introduced in 2D on polygonal cells [3, 4]. It was quickly extended to multi-material reconstruction in 3D by Ahn \& Shashkov [5] and in 2D by Dyadechko \& Shashkov [6]. Note that the latter defines a convenient error criterion and provides a convergence study of the MOF reconstruction. Another effort to extend MOF to any coordinate system was made by Anbarlooei \& Mazaheri [8] who have extended MOF to axisymmetric meshes. As MOF is a PLIC method, it is vulnerable to filaments that can not be advected. Jemison et al. [16] solved this problem by adapting MOF to filament capturing which can be done in a straightforward way by using a $n$-material reconstruction and allowing two materials to be identical in one cell. To improve the robustness of MOF, many solutions were proposed, such as the one advanced by Hill \& Shashkov [13] which consists in changing slightly the minimization problem of MOF to minimize the centroid difference on both the material and its complementary. Since the objective function of MOF contains local minima where the minimization algorithms are prone to fall into, Qing et al. [22] have proposed a method in 2D that finds all the minima of the MOF problem and selects the best one. This gain of robustness is at the expense of the runtime of the algorithm.

MOF was designed to be used in conjunction with other methods. Ahn \& Shashkov [7] proposed an interaction of MOF with an adaptive mesh refinement (AMR) strategy where the centroid difference is used as a criterion for mesh refinement. The coupling with an Arbitrary Lagrangian-Eulerian (ALE) strategy is also found among many authors $[9,11,14]$. Valuable implementation details can be found in many publications, for instance, in [10] MOF was coupled with a code based on the finite element method (FEM) and in [15] MOF is used in a compressible context. In [12] and [21], MOF is coupled with the levelset method (CLSMOF) and more recently, Kikinzon et al. [20] proposed a data structure to represent the partition of the multi-material reconstruction to simplify the interaction with other methods.

The most expensive part of the MOF reconstruction is the evaluation of the objective function and its partial derivatives at each iteration of the minimization algorithm which involves computationally intensive geometrical manipulations. To tackle this problem, Chen \& Zhang proposed analytic formulas for the partial derivatives of the objective function on convex polyhedral cells [17] and convex polygonal cells [19]. However, their methods requires a prior evaluation of the objective function with a geometric approach that still remains expensive. To completely avoid these computationally intensive geometric manipulations, another approach is to express the objective function with analytic formulas. This has been addressed by Lemoine $e t$ al. [18] in 2D for rectangular cells as we will discuss in section 2.3. In this paper, we propose a 3D extension of this method to rectangular hexahedral cells. This method can be applied to geometry tracking on any meshes composed of this kind of cells such as rectilinear grids with or without AMR.

\section{The moment-of-fluid method}

\subsection{The moment-of-fluid problem}

The MOF problem can be summarized as follows. Consider $\Omega \subset \mathbb{R}^{3}$ a portion of space, for instance a polyhedral cell and $\mathrm{M} \subset \Omega$ the location of a given material in $\Omega$, as depicted on the left of figure 1 . Where the VOF-PLIC method requires the information of the volume of material from the cell and its neighborhood, MOF embeds all the information within the cell. Besides the volume of material, the MOF reconstruction involves the centroid of $\mathrm{M}$ denoted by $\mathcal{C}(\mathrm{M})$ and defined by:

$$
\mathcal{C}(\mathrm{M})=\frac{1}{\operatorname{vol}(\mathrm{M})} \int_{\mathrm{M}} \boldsymbol{x} \mathrm{d} \boldsymbol{x}
$$

As a PLIC method, MOF approximates the location of the material M with a linear interface between the materials in $\Omega$. The resulting volume is the intersection of a half-space with $\Omega$. A half-space can be defined by two parameters, namely the outgoing unit normal $\boldsymbol{n}$ of its boundary and the shortest signed distance $d$ of its boundary to the origin. We denote $\mathrm{H}(\boldsymbol{n}, d)=\left\{\boldsymbol{x} \in \mathbb{R}^{3} \mid \boldsymbol{x} \cdot \boldsymbol{n} \leq d\right\}$ a half-space parametrized by $\boldsymbol{n}$ 
and $d$. As a result, the approximation of $\mathrm{M}$ is equal to $\mathrm{H}(\boldsymbol{n}, d) \cap \Omega$ (right of figure 1) with $\boldsymbol{n}$ and $d$ to be determined. Like the VOF methods, MOF enforces the volume conservation on its reconstruction, that is $\operatorname{vol}(H(\boldsymbol{n}, d) \cap \Omega)=\operatorname{vol}(\mathrm{M})$. In summary, the set of all the approximations of $\mathrm{M}$ in $\Omega$ can be defined by:

$$
\mathfrak{A}=\left\{\mathrm{H}(\boldsymbol{n}, d) \cap \Omega \mid \boldsymbol{n} \in \mathbb{S}^{2}, d \in \mathbb{R} \text { and } \operatorname{vol}(\mathrm{H}(\boldsymbol{n}, d) \cap \Omega)=\operatorname{vol}(\mathrm{M})\right\}
$$

The moment-of-fluid problem consists in finding the best approximation $\omega^{*} \in \mathfrak{A}$ of $\mathrm{M}$ such that its centroid is as close as possible to the centroid of the real - or reference - material location $\mathrm{M}$ denoted by $\mathcal{C}^{\star}=\mathcal{C}(\mathrm{M})$. Written in mathematical terms, the MOF problem becomes:

$$
\text { Find } \omega^{*} \in \mathfrak{A} \text { such that } \omega^{*}=\underset{\omega \in \mathfrak{A}}{\operatorname{argmin}}\left|\mathcal{C}(\omega)-\mathcal{C}^{\star}\right|^{2}
$$

Note that the solution may be not unique, but as proved in [3], the set of reference centroids for which the problem is non-unique has zero area.
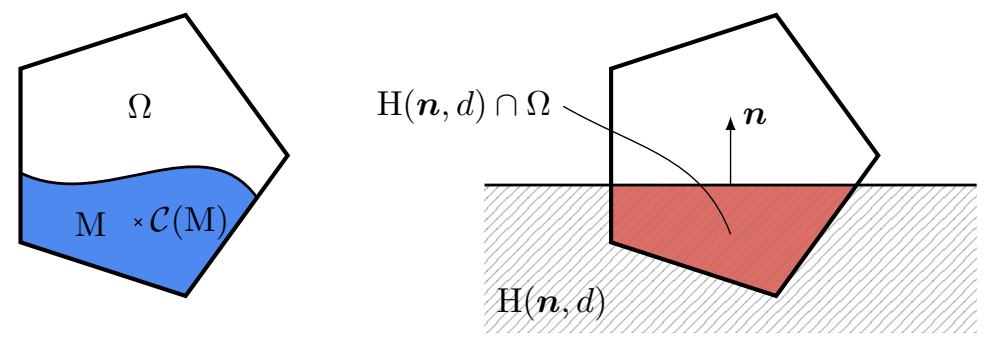

Figure 1: Illustration of the notations used to define the MOF problem.

\subsection{General method to solve the moment-of-fluid problem}

To solve the MOF problem (3), various iterative minimization algorithms have been proposed. For instance, Ahn \& Shashkov [5] use a Broyden-Fletcher-Goldfarb-Shanno (BFGS) algorithm, while other authors, such as Jemison et al. [12], prefer a Gauss-Newton method. The minimization algorithms require several evaluations of the objective function and its partial derivatives. The evaluation of the objective function requires to compute the centroid of the approximation $\mathcal{C}(\omega)$ and, therefore, the approximation $\omega$ itself. From (2), each approximation $\omega$ is defined by a direction $\boldsymbol{n}$ and a distance $d$. In practice, each step of the minimization algorithm provides a new direction $\boldsymbol{n}$ and the distance $\boldsymbol{d}$ is determined using a volume enforcement algorithm. Following Dyadechko \& Shashkov [4], such an algorithm will be referred to as a flood algorithm. It consists to find the distance $d$ of a half-space such that the volume of the intersection of this half-space and the cell is exactly equal to the prescribed volume. For instance, there is the Brent's-based method [23] or the more recent algorithm proposed by Diot \& François [24] in 2016. All these algorithms involve to compute the intersection of a plane and a polyhedron which makes this algorithm computationally intensive.

The classic strategy to evaluate the partial derivatives consists to use a finite-difference scheme. With a centered scheme, two computations of the centroid are required per direction which makes a total of four calls to the flood algorithm in 3D. Dyadechko \& Shashkov [3] proposed a formula to compute the derivative of the objective function on $2 \mathrm{D}$ polygonal cells using the prior evaluation of the objective function. This formula reduces the number of reconstructions to only one per evaluation of the objective function and its partial derivatives. Furthermore, this formula gives an exact value where the finite-difference scheme only gives an approximation. In [19], Chen \& Zhang proposed a proof of this formula and, in a second article [17], they have derived some formulas for 3D to compute the partial derivatives on convex polyhedral cells that are summarized in Appendix A.

Another approach to solve the MOF problem (3) consists to express the objective function with analytic formulas. This can be done by finding the locus of the centroids $\mathcal{C}(\omega(\boldsymbol{n}))$ for a given fixed volume and for all the normals $\boldsymbol{n}$. This locus is a closed curve in $2 \mathrm{D}$ and a closed surface in 3D. Finding a parametrization of this locus is a very difficult task on general polygonal or polyhedral cells. However, on rectangular cells, this approach has been successfully applied for the first time by Lemoine et al. [18] as presented below. 


\subsection{Analytic method to solve the moment-of-fluid problem in $2 D$ on rectangular cells}

Consider a rectangular cell $\Omega=\left[0, c_{1}\right] \times\left[0, c_{2}\right]$. In [18], Lemoine et al. proved that the locus of the centroids is the reunion of 4 arcs of parabolas and 4 arcs of hyperbolas as depicted in figure 2. To find a global parametrization of the locus of the centroids, we introduce the angle $\theta$ defined such that the normal verifies $\boldsymbol{n}(\theta)=[\cos (\theta), \sin (\theta)]$ and we denote by $\mathcal{C}^{\mathcal{V}}(\theta)$ the centroid of the approximation $\mathcal{C}(\omega(\boldsymbol{n}(\theta)))$. The MOF problem (3) can be reinterpreted as finding the angle $\theta^{*}$ such that:

$$
\theta^{*}=\underset{\theta \in[0,2 \pi]}{\operatorname{argmin}}\left|\mathcal{C}^{\mathcal{V}}(\theta)-\mathcal{C}^{\star}\right|^{2}
$$

Taking the derivative of the objective function in (4), the minimum verifies the following relation where $\boldsymbol{u} \cdot \boldsymbol{v}$ denotes the dot product between two vectors $\boldsymbol{u}$ and $\boldsymbol{v}$ :

$$
\left(\mathcal{C}^{\mathcal{V}}\left(\theta^{*}\right)-\mathcal{C}^{\star}\right) \cdot \partial_{\theta} \mathcal{C}^{\mathcal{V}}\left(\theta^{*}\right)=0
$$

It is shown that this equation is a third degree polynomial for the parabola or a fourth degree polynomial for the hyperbola. Remark that beyond finding a parametrization of the locus of the centroids, this method gives a fully analytic solution of the MOF problem.

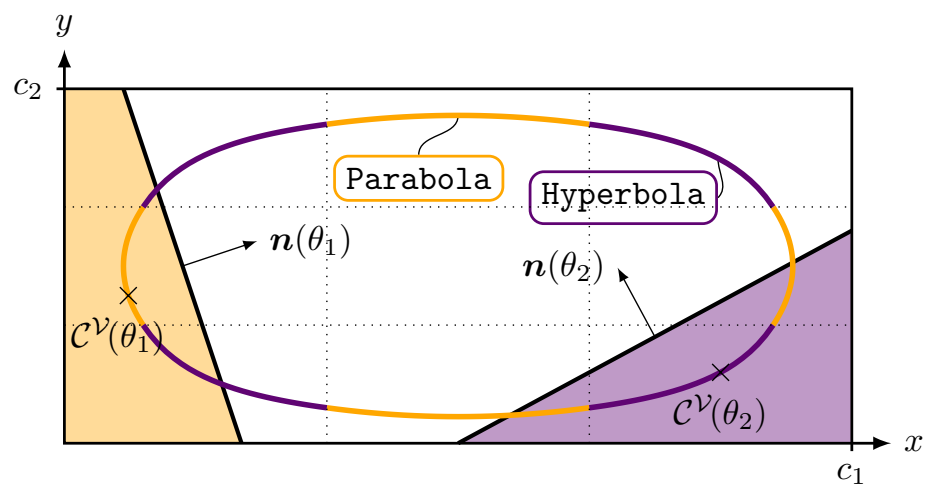

Figure 2: Illustration of the locus of the centroids (the curve) on rectangular cells for a fixed volume $\mathcal{V}$. The curve is composed of 4 arcs of parabolas and 4 arcs of hyperbola. The yellow region corresponds to an approximation shaped as a quadrangle. Its centroid is on a parabola. The purple region corresponds to an approximation shaped as a triangle. Its centroid is on a hyperbola. The purple and the yellow regions share the same area.

In this article, we have applied this methodology in 3D to rectangular hexahedrons. We will see that instead of two different configurations - triangle and quadrangle - there are five different configurations. The solution of the MOF problem verifies equation (8) which is the 3D equivalent of equation (5). In two of the five configurations, this equation reduces to two polynomial equations of fifth and twelfth degree without trivial roots, and because of the Abel-Ruffini theorem there are no general algebraic formulas to express these roots. Furthermore, in the three remaining configurations, the solution cannot be expressed as a root of a polynomial. Therefore, there is no fully analytical solution of the MOF problem (3) on rectangular hexahedron. Instead, we use a minimization algorithm where the objective function and its partial derivatives are expressed as a set of analytic formulas.

The remainder of this article is organized as follows. Section 3 describes our method as a black-box to compute the centroid and the partial derivatives for a given normal $\boldsymbol{n}$. The proof of these formulas are given in Appendix C. Section 4 presents a set of numerical tests to measure the efficiency and the robustness of our method compared to three geometric approaches. Two of them are detailed in Appendix A and Appendix B. Section 5 presents the conclusions and the future work. 


\section{Analytic method on rectangular hexahedrons}

\subsection{Description}

In the remainder of this article, we consider a rectangular hexahedron $\Omega=\left[0, c_{1}\right] \times\left[0, c_{2}\right] \times\left[0, c_{3}\right]$. Recall that MOF consists in finding the best approximation of the material location $\mathrm{M} \subset \Omega$. We define $\mathcal{V}=\operatorname{vol}(\mathrm{M})$ as the volume of $\mathrm{M}$ - or reference volume - and $\mathcal{C}^{\star}$ as its centroid - or reference centroid. From the definition of the set of the approximations $\mathfrak{A}$ in equation (2), any element of this set shares the same volume $\mathcal{V}$ as the material location M. We introduce in the following equation the normal $\boldsymbol{n}(\theta, \phi)$ of the half-space parametrized by the spherical coordinates $\theta$ and $\phi$.

$$
\boldsymbol{n}(\theta, \phi)=\left[\begin{array}{c}
\sin (\phi) \cos (\theta) \\
\sin (\phi) \sin (\theta) \\
\cos (\phi)
\end{array}\right]
$$

Consider an approximation $\omega(\boldsymbol{n}) \in \mathfrak{A}$ generated by the direction $\boldsymbol{n}$ as defined in section 2 . We denote $\mathcal{C}^{\mathcal{V}}(\theta, \phi)$ the centroid of the approximation $\mathcal{C}(\omega(\boldsymbol{n}(\theta, \phi)))$. In equation $(7)$, we define the objective function of the minimization problem (3) using the spherical coordinates.

$$
\mathcal{F}(\theta, \phi)=\left|\mathcal{C}^{\mathcal{V}}(\theta, \phi)-\mathcal{C}^{\star}\right|^{2}
$$

The minimization algorithm requires to evaluate the objective function (7) and its partial derivatives given by the following formulas:

$$
\partial_{\theta} \mathcal{F}(\theta, \phi)=2\left(\mathcal{C}^{\mathcal{V}}(\theta, \phi)-\mathcal{C}^{\star}\right) \cdot \partial_{\theta} \mathcal{C}^{\mathcal{V}}(\theta, \phi) \quad \partial_{\phi} \mathcal{F}(\theta, \phi)=2\left(\mathcal{C}^{\mathcal{V}}(\theta, \phi)-\mathcal{C}^{\star}\right) \cdot \partial_{\phi} \mathcal{C}^{\mathcal{V}}(\theta, \phi)
$$

Our method consists in finding a global parametrization of the locus of the centroids $(\theta, \phi) \mapsto \mathcal{C}^{\mathcal{V}}(\theta, \phi)$ denoted by $\mathfrak{L}^{\mathcal{V}}$ and defined in the following equation:

$$
\mathfrak{L}^{\mathcal{V}}=\left\{\mathcal{C}^{\mathcal{V}}(\theta, \phi) \mid \theta \in[-\pi, \pi] \text { and } \phi \in[0, \pi]\right\}
$$

To simplify the problem, we assume that the reference volume $\mathcal{V}$ is less than half of the volume of the cell: $\mathcal{V} \leq \operatorname{vol}(\Omega) / 2$. The case where $\mathcal{V}>\operatorname{vol}(\Omega) / 2$ can be treated by considering the complementary $\Omega \backslash \mathrm{M}$. In the remainder of this article, we will refer to the volume fraction $\chi=\mathcal{V} / \operatorname{vol}(\Omega)$ as the ratio between the reference volume and the volume of the hexahedron.

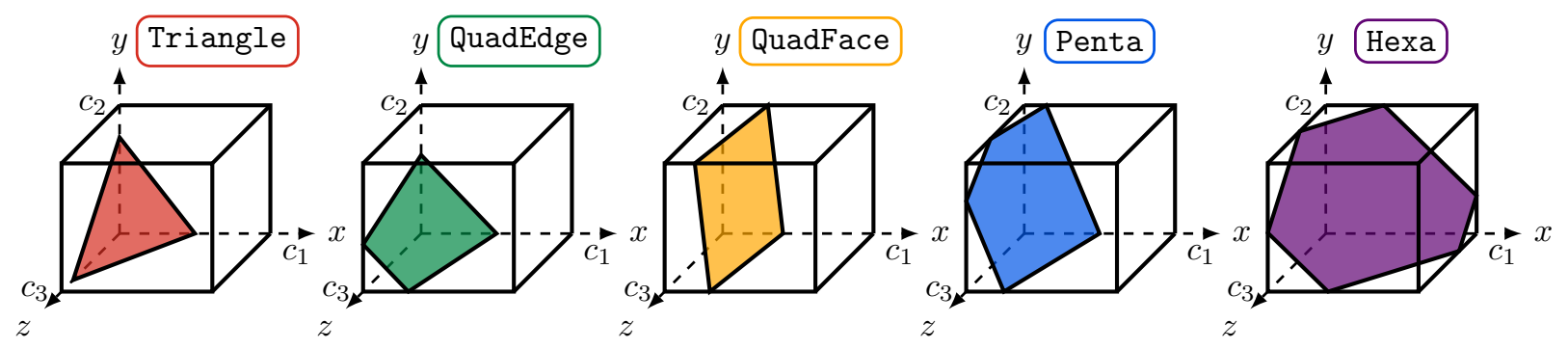

Figure 3: Five ways to intersect a rectangular hexahedron with a half-space: Triangle, QuadEdge, QuadFace, Penta and Hexa.

The shape of the locus of the centroids depends on how the half-space intersects the hexahedron, and as shown in figure 3, a half-space can intersect a hexahedron in five different ways. We denote Triangle, QuadEdge, QuadFace, Penta and Hexa the five possible configurations. We define $\mathfrak{P}$ the surface defined as the intersection of the boundary of the half-space and the hexahedron $\Omega$.

$$
\mathfrak{P}=\{\boldsymbol{x} \in \Omega \mid \boldsymbol{x} \cdot \boldsymbol{n}=d\}
$$

In the Triangle configuration, which exists if $\chi \leq \frac{1}{6}$, the half-space contains one vertex of the hexahedron and the surface $\mathfrak{P}$ is a triangle. In the QuadEdge configuration, the half-space contains one edge of the 
hexahedron and the surface is a quadrangle. In the QuadFace configuration, the half-space contains one face of the hexahedron and the surface is also a quadrangle. In the Penta configuration, the half-space contains two edges of the hexahedron and the surface $\mathfrak{P}$ is a pentagon. In the Hexa configuration, which exists if $\chi>\frac{1}{6}$, the half-space contains three edges of the hexahedron and the surface $\mathfrak{P}$ is a hexagon. Overall, the locus of the centroids is the combination of 50 configurations composed of 8 Triangle or Hexa (one per vertex), 6 QuadFace (one per face), 12 QuadEdge (one per edge) and 24 Penta (four per face). In the remainder of this article, we use the same colors as in figure 3 to depict all the configurations (refer to the web version of this article to see the colors).

Figure 4 depicts the locus of the centroids $\mathfrak{L}^{\mathcal{V}}$ for $\chi=0.1$ (left) and $\chi=0.4$ (right) where the dimensions of the hexahedron are $\left(c_{1}, c_{2}, c_{3}\right)=(1,3,2)$. We observe that the Triangle surface is only present on the locus of the left, whereas the Hexa surface is present on the locus of the right. When $\chi=\frac{1}{6}$, there are no Triangle or Hexa surfaces. The shape of the QuadEdge and Penta surfaces also depends on the volume fraction. When $\chi<\frac{1}{6}$, the boundary of the QuadEdge surface is composed of 6 curves and the boundary of the Penta surface is composed of 3 curves, while when $\chi>\frac{1}{6}$, the boundary of the QuadEdge surface is composed of 4 curves and the boundary of the Penta surface is composed of 4 curves. In the extreme case, when $\chi=\frac{1}{2}$, the QuadFace and the Hexa surfaces are the only surfaces that remain on the locus of the centroids.

In the remainder of this section, we give a parametrization of the surface $\mathfrak{L}^{\mathcal{V}}$ in terms of the spherical coordinates $(\theta, \phi)$. There is no point to provide the equations of the 50 pieces of the surface, since only 4 are sufficient to describe the whole surface (remember that the Triangle and the Hexa configurations are mutually exclusives). The symmetry of the hexahedron allows to describe the other pieces by rotations or reflections of the hexahedron, and some permutations of $\left(c_{1}, c_{2}, c_{3}\right)$.

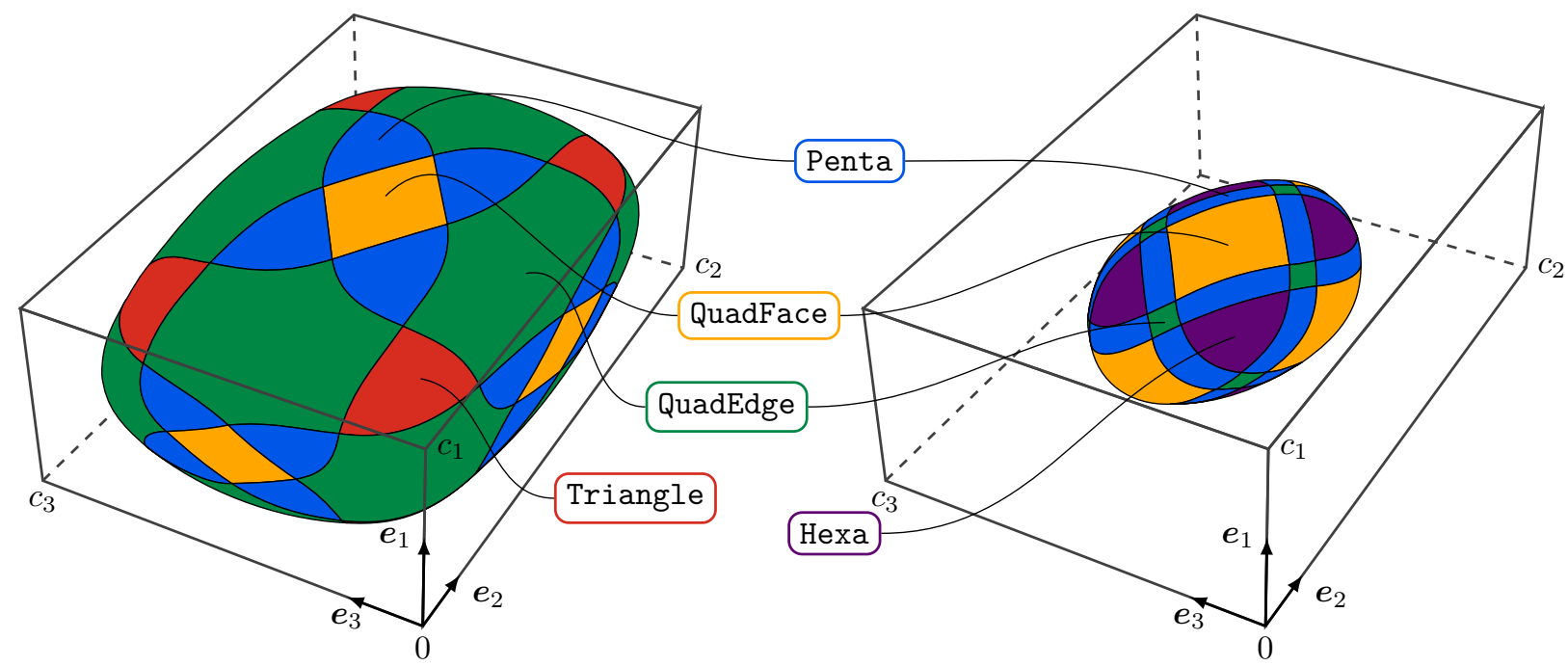

Figure 4: Locus of the centroids for the volume fractions $\chi=0.1$ (left) and $\chi=0.4$ (right). The dimensions of the hexahedron are $c_{1}=1, c_{2}=3$ and $c_{3}=2$.

\subsection{Parametrization of the locus of the centroids in the local chart}

The number of configurations can be reduced by remarking that the locus of the centroids is symmetric about the three planes $\left\{x=c_{1} / 2\right\},\left\{y=c_{2} / 2\right\}$ and $\left\{z=c_{3} / 2\right\}$. Thus, we choose to reduce the global chart $[-\pi, \pi] \times[0, \pi]$ to the region $[0, \pi / 2]^{2}$, referred to as local chart, as depicted in figure 5 . The consequence of using the definition (6) for the normal to the half-space is that the basis of the Cartesian coordinates $\left(\boldsymbol{e}_{1}, \boldsymbol{e}_{2}, \boldsymbol{e}_{3}\right)$ corresponds to $\boldsymbol{e}_{\mathbf{1}}=\boldsymbol{n}(0, \pi / 2), \boldsymbol{e}_{\mathbf{2}}=\boldsymbol{n}(\pi / 2, \pi / 2)$, and $\boldsymbol{e}_{\mathbf{3}}=\boldsymbol{n}(\theta, 0)$ for all $\theta$. In terms of surface, the considered region corresponds to the part of the locus $\mathfrak{L}^{\mathcal{V}}$ such that $x_{i} \in\left[0, c_{i} / 2\right]$ for $i \in\{1,2,3\}$. Note that the origin of the coordinates corresponds to one of the vertices of the hexahedron as depicted in figure 4. 
Figure 5 represents the local charts in $\theta$ and $\phi$ corresponding to the locus of the centroids for the volume fractions $\chi=0.1$ (left) and $\chi=0.25$ (right). With this parametrization, the distances and the areas of the configurations are highly distorted when $\phi$ approaches 0 . At the limit, the line $\phi=0$ maps to a single point which corresponds to the south pole of the locus (remember that the centroid is inside the half-space, in the opposite direction of the normal like in 2D as depicted in figure 2). In these local charts, 10 pieces are still involved in the description of the locus $\mathfrak{L}^{\mathcal{V}}$.

As shown in figure 5, the local chart is decomposed in three regions — Left, Right, and Bottom arranged around a Triangle (or a Hexa if $\chi>\frac{1}{6}$ ). Each region contains exactly one Penta, one QuadEdge, and one QuadFace. These regions are delimited by the curves $\phi_{\star}^{\lim }$ defined in section 3.2.1. We will see in section 3.3.1 that any coordinates $(\theta, \phi)$ in the global chart can be transformed to the local chart by some symmetries as depicted in figure 6 .
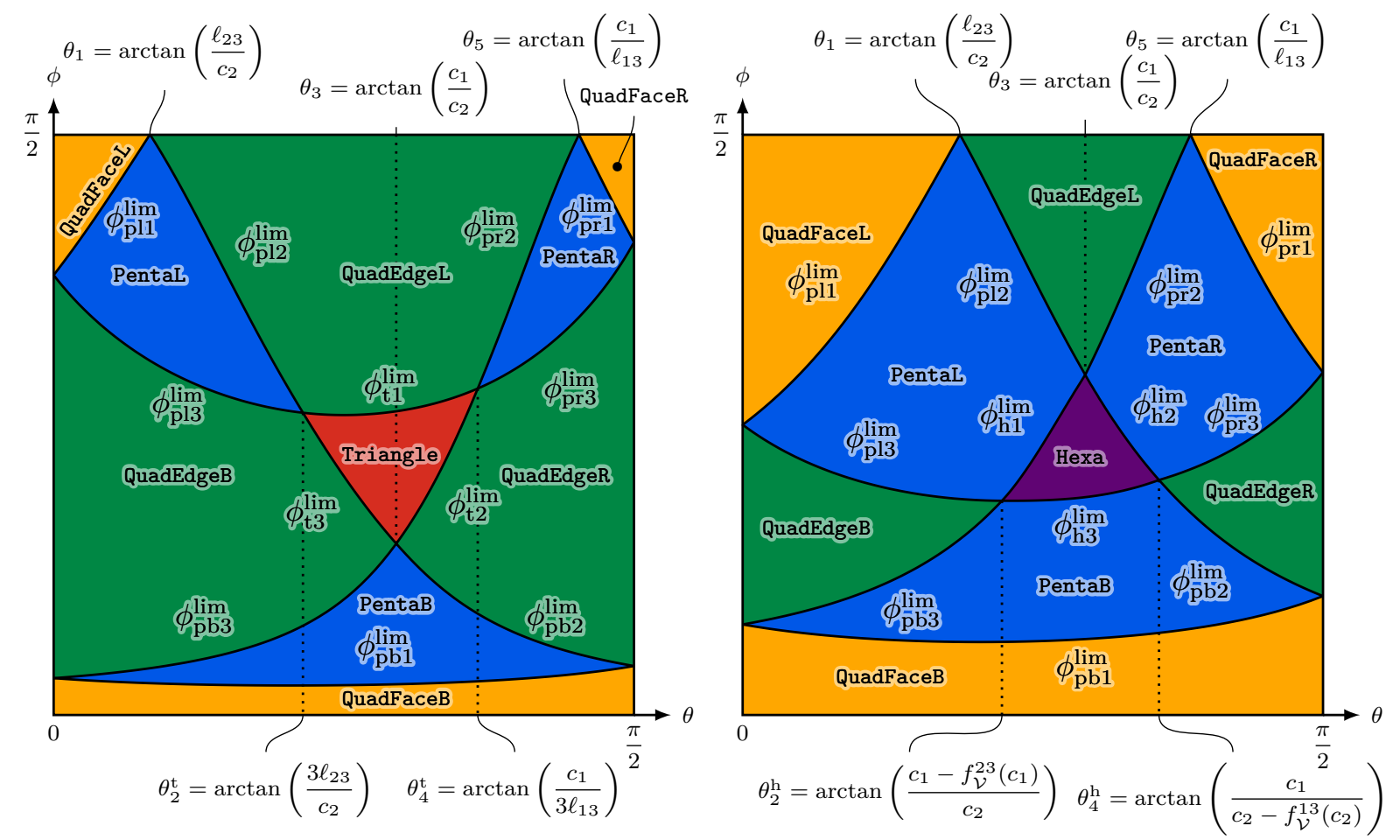

Figure 5: Local charts of the locus of the centroids reduced to $[0, \pi / 2]^{2}$ for the volume fractions $\chi=0.1$ (left) and $\chi=0.25$ (right). The dimensions of the hexahedron are $c_{1}=2, c_{2}=1.5$ and $c_{3}=1$. Note that Triangle and Hexa are mutually exclusive.

In the remainder of this section, we present how to compute the centroid and its partial derivatives from any point of the local chart. First, in section 3.2.1, we present the equations of the limit curves $\phi_{\star}^{\lim }$. Then, in section 3.2.2, we present the parametrization of the centroid and its partial derivatives in the local chart.

\subsubsection{Limits of the local chart}

We introduce the notations in equation (11) to simplify the formulas given in this section. The proof of the formulas presented in this section is given in Appendix C.

$$
\begin{gathered}
\ell_{i j}=\frac{2 \mathcal{V}}{c_{i} c_{j}} \quad f_{\mathcal{V}}^{i j}(x)=\frac{-x+\sqrt{12 \ell_{i j} x-3 x^{2}}}{2} \quad T_{2}^{l}=c_{2} \tan (\theta) \quad T_{3}^{l}=c_{3} \cot (\phi) \sec (\theta) \\
T_{2}^{r}=c_{3} \cot (\phi) \csc (\theta) \quad T_{3}^{r}=c_{1} \cot (\theta) \quad T_{2}^{b}=c_{1} \tan (\phi) \cos (\theta) \quad T_{3}^{b}=c_{2} \tan (\phi) \sin (\theta)
\end{gathered}
$$

In the Triangle configuration, the limit curves are given by equations (12). Note that $\operatorname{arccot}(x)=$ $\frac{\pi}{2}-\arctan (x)$ when $x>0$, which is verified in all our formulas. We recommend to use the formulas with 
$\arctan$ instead of $\operatorname{arccot}$ or check that $\operatorname{arccot}(0)=\frac{\pi}{2}$ to machine precision.

$$
\phi_{\mathrm{t} 1}^{\lim }(\theta)=\operatorname{arccot}\left(\sqrt{3 \ell_{23} T_{2}^{l}} \frac{\cos (\theta)}{c_{3}}\right) \quad \phi_{\mathrm{t} 2}^{\lim }(\theta)=\operatorname{arccot}\left(\frac{\left(T_{3}^{r}\right)^{2}}{3 \ell_{13}} \frac{\sin (\theta)}{c_{3}}\right) \quad \phi_{\mathrm{t} 3}^{\lim }(\theta)=\operatorname{arccot}\left(\frac{\left(c_{2} \sin (\theta)\right)^{2}}{3 \ell_{12}\left(c_{1} \cos (\theta)\right)}\right)
$$

In the PentaL configuration, the limit curves are given by the equations (13a), (13b), and (13c).

$$
\begin{aligned}
& \phi_{\mathrm{p} 11}^{\lim }(\theta)=\operatorname{arccot}\left(\left(\ell_{23}-T_{2}^{l}\right) \frac{\cos (\theta)}{c_{3}}\right) \\
& \phi_{\mathrm{p} 12}^{\lim }(\theta)=\operatorname{arccot}\left(\left(T_{2}^{l}-f_{\mathcal{V}}^{23}\left(T_{2}^{l}\right)\right) \frac{\cos (\theta)}{c_{3}}\right) \\
& \phi_{\mathrm{p} 13}^{\lim }(\theta)=\operatorname{arccot}\left(\left(\ell_{23}+T_{2}^{l}+\sqrt{\left(\ell_{23}+T_{2}^{l}\right)^{2}-\frac{4}{3}\left(T_{2}^{l}\right)^{2}}\right) \frac{\cos (\theta)}{2 c_{3}}\right)
\end{aligned}
$$

In the PentaR configuration, the limit curves are given by the equations (14a), (14b), and (14c).

$$
\begin{aligned}
& \phi_{\mathrm{pr} 1}^{\lim _{1}}(\theta)=\operatorname{arccot}\left(\left(\ell_{13}-T_{3}^{r}\right) \frac{\sin (\theta)}{c_{3}}\right) \\
& \phi_{\mathrm{pr} 2}^{\lim _{2}(\theta)}=\operatorname{arccot}\left(\left(T_{3}^{r}-f_{\mathcal{V}}^{13}\left(T_{3}^{r}\right)\right) \frac{\sin (\theta)}{c_{3}}\right) \\
& \phi_{\mathrm{pr} 3}^{\lim _{3}}(\theta)=\operatorname{arccot}\left(\left(\ell_{13}+T_{3}^{r}+\sqrt{\left(\ell_{13}+T_{3}^{r}\right)^{2}-\frac{4}{3}\left(T_{3}^{r}\right)^{2}}\right) \frac{\sin (\theta)}{2 c_{3}}\right)
\end{aligned}
$$

In the PentaB configuration, the limit curves are given by the equations (15a), (15b), and (15c).

$$
\begin{aligned}
& \phi_{\mathrm{pb} 1}^{\lim }(\theta)=\operatorname{arccot}\left(\left(c_{2} \sin (\theta)+c_{1} \cos (\theta)\right) \frac{1}{\ell_{12}}\right) \\
& \phi_{\mathrm{pb} 2}^{\lim }(\theta)=\operatorname{arccot}\left(\left(c_{2} \sin (\theta)-c_{1} \cos (\theta)+\frac{\left(c_{1} \cos (\theta)\right)^{2}}{3 c_{2} \sin (\theta)}\right) \frac{1}{\ell_{12}}\right) \\
& \phi_{\mathrm{pb} 3}^{\lim }(\theta)=\operatorname{arccot}\left(\left(c_{1} \cos (\theta)-c_{2} \sin (\theta)+\frac{\left(c_{2} \sin (\theta)\right)^{2}}{3 c_{1} \cos (\theta)}\right) \frac{1}{\ell_{12}}\right)
\end{aligned}
$$

In the Hexa configuration, the limit curves are given by equations (16a), (16b), and (16c).

$$
\begin{aligned}
& \phi_{\mathrm{h} 1}^{\lim }(\theta)=\operatorname{arccot}\left(\left(c_{1}-2 \sqrt{\ell_{23} T_{2}^{l}} \cos \left(\frac{1}{3} \arccos \left(\frac{3 c_{1}\left(c_{1}-\ell_{23}-T_{2}^{l}\right)+\left(T_{2}^{l}\right)^{2}}{2 \ell_{23} \sqrt{\ell_{23} T_{2}^{l}}}\right)+\frac{4 \pi}{3}\right)\right) \frac{\cos (\theta)}{c_{3}}\right) \\
& \phi_{\mathrm{h} 2}^{\lim }(\theta)=\operatorname{arccot}\left(\left(c_{2}-2 \sqrt{\ell_{13} T_{3}^{r}} \cos \left(\frac{1}{3} \arccos \left(\frac{3 c_{2}\left(c_{2}-\ell_{13}-T_{3}^{r}\right)+\left(T_{3}^{r}\right)^{2}}{2 \ell_{13} \sqrt{\ell_{13} T_{3}^{r}}}\right)+\frac{4 \pi}{3}\right)\right) \frac{\sin (\theta)}{c_{3}}\right) \\
& \phi_{\mathrm{h} 3}^{\lim }(\theta)=\operatorname{arccot}\left(\left(c_{1}+T_{2}^{l}+2 \sqrt{\left(2 c_{1}-\ell_{23}\right) T_{2}^{l}} \cos \left(\frac{1}{3} \arccos \left(\frac{3\left(c_{1}-\ell_{23}\right)\left(c_{1}+T_{2}^{l}\right)}{2\left(2 c_{1}-\ell_{23}\right) \sqrt{\left(2 c_{1}-\ell_{23}\right) T_{2}^{l}}}\right)+\frac{4 \pi}{3}\right)\right) \frac{\cos (\theta)}{c_{3}}\right)
\end{aligned}
$$

\subsubsection{Centroids and derivatives in spherical coordinates in the local chart}

The parametrization of the centroid locus $\mathcal{C}^{\mathcal{V}}(\theta, \phi)$ is defined as a piecewise function on each configuration. Inside the local chart, it is given by equations (17) to (22). As in section 3.2.1, the notations defined in (11) are used to simplify the formulas in this section.

In the Triangle configuration, the parametrization is given by equation (17) which is defined for $\chi<\frac{1}{6}$.

$$
\mathcal{C}_{\mathrm{t}}^{\mathcal{V}}(\theta, \phi)=\frac{1}{4}\left(3 \ell_{23} T_{2}^{l} T_{3}^{l}\right)^{1 / 3}\left[\begin{array}{c}
1 \\
c_{2} / T_{2}^{l} \\
c_{3} / T_{3}^{l}
\end{array}\right]
$$


In the QuadFace configurations, the parametrizations are given by:

$$
\begin{array}{ll}
\text { QuadFaceL } & \mathcal{C}_{\mathrm{qf}}^{\mathcal{V}}(\theta, \phi)=\frac{1}{12 \ell_{23}}\left[\begin{array}{c}
3\left(\ell_{23}\right)^{2}+\left(T_{2}^{l}\right)^{2}+\left(T_{3}^{l}\right)^{2} \\
2 c_{2}\left(3 \ell_{23}-T_{2}^{l}\right) \\
2 c_{3}\left(3 \ell_{23}-T_{3}^{l}\right)
\end{array}\right] \\
\text { QuadFaceR } & \mathcal{C}_{\mathrm{qfr}}^{\mathcal{V}}(\theta, \phi)=\frac{1}{12 \ell_{13}}\left[\begin{array}{c}
2 c_{1}\left(3 \ell_{13}-T_{3}^{r}\right) \\
3\left(\ell_{13}\right)^{2}+\left(T_{2}^{r}\right)^{2}+\left(T_{3}^{r}\right)^{2} \\
2 c_{3}\left(3 \ell_{13}-T_{2}^{r}\right)
\end{array}\right] \\
\text { QuadFaceB } & \mathcal{C}_{\mathrm{qfb}}^{\mathcal{V}}(\theta, \phi)=\frac{1}{12 \ell_{12}}\left[\begin{array}{c}
2 c_{1}\left(3 \ell_{12}-T_{2}^{b}\right) \\
2 c_{2}\left(3 \ell_{12}-T_{3}^{b}\right) \\
3\left(\ell_{12}\right)^{2}+\left(T_{2}^{b}\right)^{2}+\left(T_{3}^{b}\right)^{2}
\end{array}\right]
\end{array}
$$

In the QuadEdge configurations the parametrizations are given by:

$$
\begin{aligned}
& \text { QuadEdgeL } \quad \mathcal{C}_{\mathrm{qel}}^{\mathcal{V}}(\theta, \phi)=\frac{1}{108 \ell_{23}}\left[\begin{array}{c}
\frac{1}{T_{2}^{l}}\left(6 \ell_{23} T_{2}^{l}+\left(T_{3}^{l}\right)^{2}\right) \mathcal{X}_{\mathrm{qel}} \\
\frac{c_{2}}{\left(T_{2}^{l}\right)^{2}}\left(6 \ell_{23} T_{2}^{l}+\left(T_{3}^{l}\right)^{2}\right) \mathcal{X}_{\mathrm{qel}} \\
3 c_{3}\left(18 \ell_{23}-\frac{T_{3}^{l}}{T_{2}^{l}} \mathcal{X}_{\mathrm{qel}}\right)
\end{array}\right] \quad \mathcal{X}_{\mathrm{qel}}=\sqrt{36 \ell_{23} T_{2}^{l}-3\left(T_{3}^{l}\right)^{2}} \\
& \text { QuadEdgeR } \quad \mathcal{C}_{\mathrm{qer}}^{\mathcal{V}}(\theta, \phi)=\frac{1}{108 \ell_{13}}\left[\begin{array}{c}
3 c_{1}\left(18 \ell_{13}-\frac{T_{3}^{r}}{T_{2}^{r}} \mathcal{X}_{\mathrm{qer}}\right) \\
\frac{1}{T_{2}^{r}}\left(6 \ell_{13} T_{2}^{r}+\left(T_{3}^{r}\right)^{2}\right) \mathcal{X}_{\mathrm{qer}} \\
\frac{c_{3}}{\left(T_{2}^{r}\right)^{2}}\left(6 \ell_{13} T_{2}^{r}+\left(T_{3}^{r}\right)^{2}\right) \mathcal{X}_{\mathrm{qer}}
\end{array}\right] \quad \mathcal{X}_{\mathrm{qer}}=\sqrt{36 \ell_{13} T_{2}^{r}-3\left(T_{3}^{r}\right)^{2}} \\
& \text { QuadEdgeB } \quad \mathcal{C}_{\mathrm{qeb}}^{\mathcal{V}}(\theta, \phi)=\frac{1}{108 \ell_{12}}\left[\begin{array}{c}
\frac{c_{1}}{\left(T_{2}^{b}\right)^{2}}\left(6 \ell_{12} T_{2}^{b}+\left(T_{3}^{b}\right)^{2}\right) \mathcal{X}_{\mathrm{qeb}} \\
3 c_{2}\left(18 \ell_{12}-\frac{T_{3}^{b}}{T_{2}^{b}} \mathcal{X}_{\mathrm{qeb}}\right) \\
\frac{1}{T_{2}^{b}}\left(6 \ell_{12} T_{2}^{b}+\left(T_{3}^{b}\right)^{2}\right) \mathcal{X}_{\mathrm{qeb}}
\end{array}\right] \quad \mathcal{X}_{\mathrm{qeb}}=\sqrt{36 \ell_{12} T_{2}^{b}-3\left(T_{3}^{b}\right)^{2}}
\end{aligned}
$$

In the Penta configurations, the parametrizations are given by:

$$
\begin{array}{ll}
\mathcal{F}_{\mathrm{p}}^{0}(x, y)=2\left(x^{2}+y^{2}\right)+3 x y & \mathcal{F}_{\mathrm{p}}^{1}\left(x, y, \ell_{i j}\right)=3 \sqrt{2 x y}\left(3(x+y)+\ell_{i j}\right) \\
\mathcal{F}_{\mathrm{p}}^{2}\left(x, y, \ell_{i j}\right)=6 \ell_{i j}-(4 x+3 y) & \mathcal{F}_{\mathrm{p}}^{3}\left(x, y, \ell_{i j}\right)=6 \sqrt{\frac{y}{2 x}}\left(\ell_{i j}-(5 x+y)\right)
\end{array}
$$

PentaL

$$
\mathcal{C}_{\mathrm{pl}}^{\mathcal{V}}(\theta, \phi)=\frac{1}{6 \ell_{23}}\left[\begin{array}{c}
\mathcal{F}_{\mathrm{p}}^{0}\left(T_{2}^{l}, T_{3}^{l}\right)+\mathcal{F}_{\mathrm{p}}^{1}\left(T_{2}^{l}, T_{3}^{l}, \ell_{23}\right) \mathcal{X}_{\mathrm{pl}}+24 T_{2}^{l} T_{3}^{l}\left(\mathcal{X}_{\mathrm{pl}}\right)^{2} \\
c_{2}\left(\mathcal{F}_{\mathrm{p}}^{2}\left(T_{2}^{l}, T_{3}^{l}, \ell_{23}\right)+\mathcal{F}_{\mathrm{p}}^{3}\left(T_{2}^{l}, T_{3}^{l}, \ell_{23}\right) \mathcal{X}_{\mathrm{pl}}-24 T_{3}^{l}\left(\mathcal{X}_{\mathrm{pl}}\right)^{2}\right) \\
c_{3}\left(\mathcal{F}_{\mathrm{p}}^{2}\left(T_{3}^{l}, T_{2}^{l}, \ell_{23}\right)+\mathcal{F}_{\mathrm{p}}^{3}\left(T_{3}^{l}, T_{2}^{l}, \ell_{23}\right) \mathcal{X}_{\mathrm{pl}}-24 T_{2}^{l}\left(\mathcal{X}_{\mathrm{pl}}\right)^{2}\right)
\end{array}\right]
$$

$$
\begin{gathered}
\mathcal{X}_{\mathrm{pl}}=\cos \left(\frac{1}{3} \arccos \left(\frac{3\left(T_{2}^{l}+T_{3}^{l}-\ell_{23}\right)}{4 \sqrt{2 T_{2}^{l} T_{3}^{l}}}\right)+\frac{4 \pi}{3}\right) \\
\mathcal{C}_{\mathrm{pr}}^{\mathcal{V}}(\theta, \phi)=\frac{1}{6 \ell_{13}}\left[\begin{array}{c}
c_{1}\left(\mathcal{F}_{\mathrm{p}}^{2}\left(T_{3}^{r}, T_{2}^{r}, \ell_{13}\right)+\mathcal{F}_{\mathrm{p}}^{3}\left(T_{3}^{r}, T_{2}^{r}, \ell_{13}\right) \mathcal{X}_{\mathrm{pr}}-24 T_{2}^{r}\left(\mathcal{X}_{\mathrm{pr}}\right)^{2}\right) \\
\mathcal{F}_{\mathrm{p}}^{0}\left(T_{2}^{r}, T_{3}^{r}\right)+\mathcal{F}_{\mathrm{p}}^{1}\left(T_{2}^{r}, T_{3}^{r}, \ell_{13}\right) \mathcal{X}_{\mathrm{pr}}+24 T_{2}^{r} T_{3}^{r}\left(\mathcal{X}_{\mathrm{pr}}\right)^{2} \\
c_{3}\left(\mathcal{F}_{\mathrm{p}}^{2}\left(T_{2}^{r}, T_{3}^{r}, \ell_{13}\right)+\mathcal{F}_{\mathrm{p}}^{3}\left(T_{2}^{r}, T_{3}^{r}, \ell_{13}\right) \mathcal{X}_{\mathrm{pr}}-24 T_{3}^{r}\left(\mathcal{X}_{\mathrm{pr}}\right)^{2}\right)
\end{array}\right]
\end{gathered}
$$

PentaR

$$
\mathcal{X}_{\mathrm{pr}}=\cos \left(\frac{1}{3} \arccos \left(\frac{3\left(T_{2}^{r}+T_{3}^{r}-\ell_{13}\right)}{4 \sqrt{2 T_{2}^{r} T_{3}^{r}}}\right)+\frac{4 \pi}{3}\right)
$$$$
\mathcal{C}_{\mathrm{pb}}^{\mathcal{V}}(\theta, \phi)=\frac{1}{6 \ell_{12}}\left[\begin{array}{c}
c_{1}\left(\mathcal{F}_{\mathrm{p}}^{2}\left(T_{2}^{b}, T_{3}^{b}, \ell_{12}\right)+\mathcal{F}_{\mathrm{p}}^{3}\left(T_{2}^{b}, T_{3}^{b}, \ell_{12}\right) \mathcal{X}_{\mathrm{pb}}-24 T_{3}^{b}\left(\mathcal{X}_{\mathrm{pb}}\right)^{2}\right) \\
c_{2}\left(\mathcal{F}_{\mathrm{p}}^{2}\left(T_{3}^{b}, T_{2}^{b}, \ell_{12}\right)+\mathcal{F}_{\mathrm{p}}^{3}\left(T_{3}^{b}, T_{2}^{b}, \ell_{12}\right) \mathcal{X}_{\mathrm{pb}}-24 T_{2}^{b}\left(\mathcal{X}_{\mathrm{pb}}\right)^{2}\right) \\
\mathcal{F}_{\mathrm{p}}^{0}\left(T_{2}^{b}, T_{3}^{b}\right)+\mathcal{F}_{\mathrm{p}}^{1}\left(T_{2}^{b}, T_{3}^{b}, \ell_{12}\right) \mathcal{X}_{\mathrm{pb}}+24 T_{2}^{b} T_{3}^{b}\left(\mathcal{X}_{\mathrm{pb}}\right)^{2}
\end{array}\right]
$$

$$
\mathcal{X}_{\mathrm{pb}}=\cos \left(\frac{1}{3} \arccos \left(\frac{3\left(T_{2}^{b}+T_{3}^{b}-\ell_{12}\right)}{4 \sqrt{2 T_{2}^{b} T_{3}^{b}}}\right)+\frac{4 \pi}{3}\right)
$$


In the Hexa configuration, the parametrization is given by the following equation which is defined for $\chi \geq \frac{1}{6}$ :

$$
\begin{aligned}
& \mathcal{C}_{\mathrm{h}}^{\mathcal{V}}(\theta, \phi)=\frac{1}{96 \ell_{23}}\left[\begin{array}{c}
\frac{1}{T_{2}^{l}} \mathcal{F}_{\mathrm{h}}^{0}\left(c_{1}, T_{2}^{l}, T_{3}^{l}\right)-24\left(c_{1}-\ell_{23}\right)\left(\sqrt{T_{4}^{l}} \mathcal{X}_{\mathrm{h}}+2 c_{1}\right)+\frac{12\left(\mathcal{X}_{\mathrm{h}}\right)^{2}}{T_{2}^{l} T_{3}^{l}} \mathcal{F}_{\mathrm{h}}^{1}\left(c_{1}, T_{2}^{l}, T_{3}^{l}, T_{4}^{l}\right) \\
c_{2}\left(\frac{1}{\left(T_{2}^{l}\right)^{2}} \mathcal{F}_{\mathrm{h}}^{0}\left(T_{2}^{l}, c_{1}, T_{3}^{l}\right)-24\left(c_{1}-\ell_{23}\right)\left(\frac{\sqrt{T_{4}^{l}}}{T_{2}^{l}} \mathcal{X}_{\mathrm{h}}+2\right)+\frac{12\left(\mathcal{X}_{\mathrm{h}}\right)^{2}}{\left(T_{2}^{l}\right)^{2} T_{3}^{l}} \mathcal{F}_{\mathrm{h}}^{1}\left(T_{2}^{l}, c_{1}, T_{3}^{l}, T_{4}^{l}\right)\right) \\
c_{3}\left(\frac{1}{T_{2}^{l}} \mathcal{F}_{\mathrm{h}}^{2}\left(c_{1}, T_{2}^{l}, T_{3}^{l}\right)-24\left(c_{1}-\ell_{23}\right)\left(\frac{\sqrt{T_{4}^{l}}}{T_{3}^{l}} \mathcal{X}_{\mathrm{h}}+2\right)+\frac{12\left(\mathcal{X}_{\mathrm{h}}\right)^{2}}{T_{2}^{l}\left(T_{3}^{l}\right)^{2}} \mathcal{F}_{\mathrm{h}}^{l}\left(T_{3}^{l}, c_{1}, T_{2}^{l}, T_{4}^{l}\right)\right)
\end{array}\right] \\
& \mathcal{F}_{\mathrm{h}}^{0}(x, y, z)=\frac{(x-y)^{3}(3 x+y)}{z}-\left(8 x^{3}+z^{3}-4 y\left(y^{2}+z^{2}+9 x^{2}\right)+6 z\left(y^{2}-x^{2}\right)\right) \\
& \mathcal{F}_{\mathrm{h}}^{1}(x, y, z, t)=\left(2\left(x^{2}-(y-z)^{2}\right)-t\right) t \quad \mathcal{F}_{\mathrm{h}}^{2}(x, y, z)=-\frac{(x-y)^{4}}{z^{2}}+6\left((x+y)^{2}+4 x y\right)-8(x+y) z+3 z^{2} \\
& T_{4}^{l}=4 c_{1} T_{3}^{l}-\left(c_{1}-T_{2}^{l}+T_{3}^{l}\right)^{2} \quad \mathcal{X}_{\mathrm{h}}=\cos \left(\frac{1}{3} \arccos \left(\frac{6\left(c_{1}-\ell_{23}\right) T_{2}^{l} T_{3}^{l}}{\left(T_{4}^{l}\right)^{\frac{3}{2}}}\right)+\frac{4 \pi}{3}\right)
\end{aligned}
$$

The partial derivatives can be easily computed for the Triangle, the QuadEdge, and QuadFace configurations by applying the chain rule to the aforementioned formulas. Since these are essentially polynomial functions or square roots, we do not provide them in this article. However, due to the complexity of the expressions of the centroid of the Penta and Hexa configurations, we prefer to use the analytical derivatives given by Chen \& Zhang presented in Appendix A. Their formula requires the coordinates of the vertices of the interface $\mathfrak{P}$ given in a compatible order with the outgoing normal as presented in figure A.8. For these configurations, the coordinates of the vertices represented in figures C.15 and C.16 are given in equations (C.36) and (C.45).

\subsection{Algorithm to evaluate the objective function and its partial derivatives}

\subsubsection{Introduction}

In the former section we presented the formulas to compute the centroid and its partial derivatives in the local chart. In this section we will present the computation of the objective function and its gradient in the global chart that are required by the minimization algorithm. We denote $\mathcal{T}$ as the application that maps the coordinates from the global chart $(\theta, \phi)$ to the local chart $(\tilde{\theta}, \tilde{\phi})$ and is defined by:

$$
(\tilde{\theta}, \tilde{\phi})=\mathcal{T}(\theta, \phi)
$$

From figure 4, it is clear that any point in the global chart can be transformed to the local chart with the following operations. First, if the point lies in the northern hemisphere, it is transformed to the southern hemisphere by a reflection with respect to the plane $\left\{z=\frac{c_{3}}{2}\right\}$. The point is then mapped into the local chart with one or two quarter turns around the third axis. To keep track of these transformations during the algorithm we chose to use a set of signs denoted by $s=\left\{s_{1}, s_{2}, s_{3}\right\}$. For the set of signs, each element verifies the relation $\mathrm{s}_{i}= \pm 1$. To illustrate these notations, consider the following three examples. A reflection with respect to the plane $\left\{z=\frac{c_{3}}{2}\right\}$ is represented by $s=\{1,1,-1\}$. A quarter turn of $+\frac{\pi}{2}$ around the third axis is represented $\mathbf{s}=\{-1,1,1\}$.

From these definitions, the transformation $\mathcal{T}$ is found by solving the following equation for $\tilde{\theta}$ and $\tilde{\phi}$ where $\boldsymbol{n}$ is defined in equation (6).

$$
\boldsymbol{n}(\tilde{\theta}, \tilde{\phi})=\mathrm{s} \circ(\sigma \cdot \boldsymbol{n}(\theta, \phi))
$$

The $\circ$ symbol represents a term by term multiplication and $\sigma$ is the permutation of the two first axes made in the transformation from the global chart to the local chart. The later can only be equal to the permutation of the first and the second coordinates denoted by $\tau_{12}$ when the rotation is a quarter turn or the identity permutation denoted by id otherwise. It is easy to check that the set of signs can be used to encode the permutation. Thus, $\sigma=$ id if $\mathrm{s}_{1} \mathrm{~s}_{2}>0$ and $\sigma=\tau_{12}$ if $\mathrm{s}_{1} \mathrm{~s}_{2}<0$. 
Since the transformation of the hexahedron preserves the distances, the objective function can be written in the local chart:

$$
\mathcal{F}(\theta, \phi)=\left|\mathcal{C}^{\mathcal{V}}(\theta, \phi)-\mathcal{C}^{\star}\right|^{2}=\left|\mathcal{C}^{\mathcal{V}}(\mathcal{T}(\theta, \phi))-\mathcal{C}_{\text {loc }}^{\star}\right|^{2}=\mathcal{F}_{\text {loc }}(\mathcal{T}(\theta, \phi))
$$

where $\mathcal{C}_{\text {loc }}^{\star}$ denotes the reference centroid transformed to the local chart. It is obtained by applying the permutation to the coordinates of the reference centroid $\mathcal{C}_{\text {loc }}^{\star} \leftarrow \sigma \cdot \mathcal{C}^{\star}$ and then by applying the reflection $\left(\mathcal{C}_{\text {loc }}^{\star}\right)_{i} \leftarrow c_{i}-\left(\mathcal{C}_{\text {loc }}^{\star}\right)_{i}$ if $s_{i}=-1$. As a result, the value of the objective function in the global chart is equal to the value of the objective function in the local chart. The computation of the gradient in the global chart is a little more tricky and is given by $\nabla_{(\theta, \phi)} \mathcal{F}=\left[\nabla_{(\theta, \phi)} \mathcal{T}\right]^{T} \nabla_{(\tilde{\theta}, \tilde{\phi})} \mathcal{F}_{\text {loc }}$. For the considered transformation, it can be shown that:

$$
\left[\begin{array}{c}
\partial_{\theta} \mathcal{F}(\theta, \phi) \\
\partial_{\phi} \mathcal{F}(\theta, \phi)
\end{array}\right]=\left[\begin{array}{c}
\partial_{\tilde{\theta}} \mathcal{F}_{\mathrm{loc}}(\tilde{\theta}, \tilde{\phi}) \\
\mathrm{s}_{3} \partial_{\tilde{\phi}} \mathcal{F}_{\mathrm{loc}}(\tilde{\theta}, \tilde{\phi})
\end{array}\right]
$$

We propose a 2-stage algorithm to evaluate the objective function and its gradient. The first stage consists to find $\sigma$ and $\mathrm{s}$ to transform the given coordinates $(\theta, \phi)$ from the global chart to the local chart $(\tilde{\theta}, \tilde{\phi})$. The second stage consists to evaluate the centroid, its partial derivatives, and the gradient of the objective function in the local and global charts.

\subsubsection{Stage 1: Transformation to local chart}

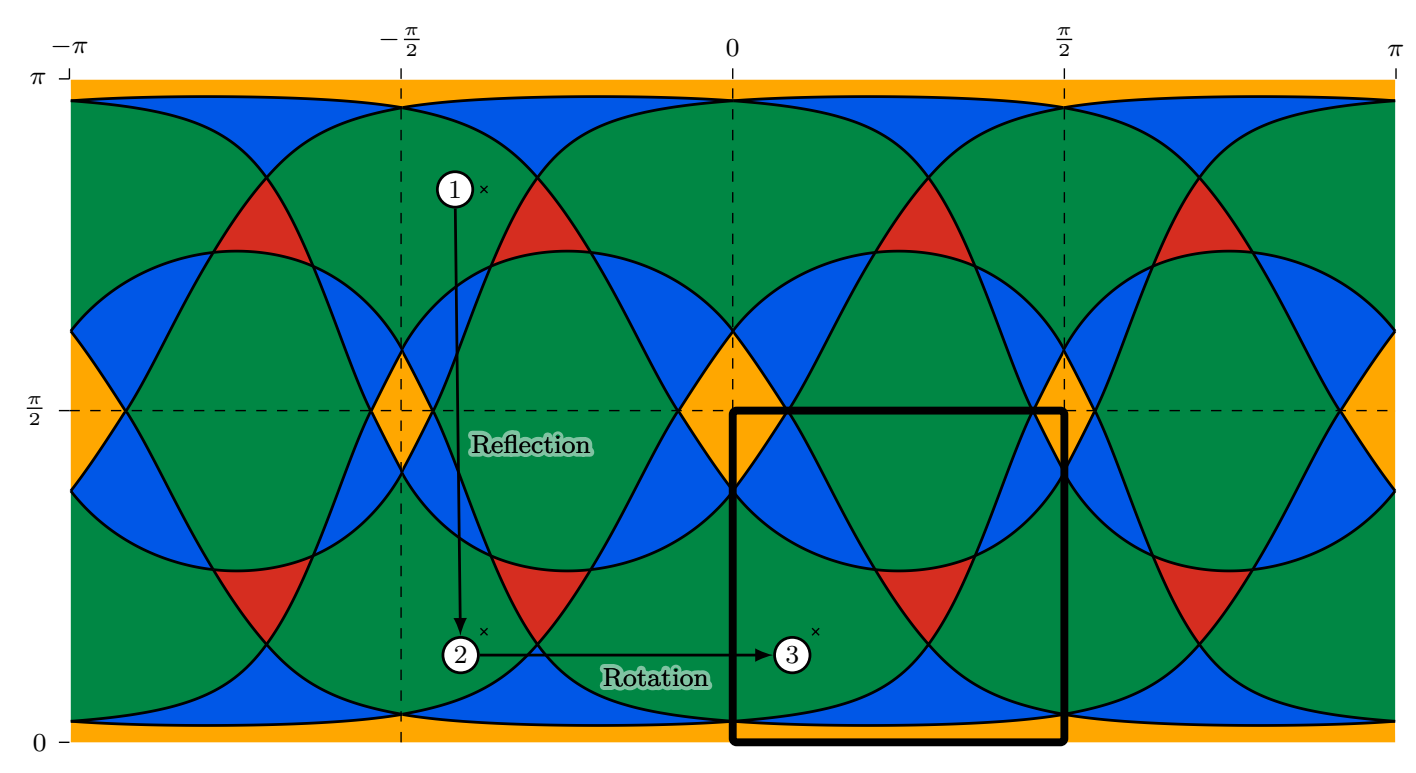

Figure 6: Illustration of the transformation of a point $(\theta, \phi)$ from the global chart to the reference chart. (1) The initial point. (2) The coordinate $\phi$ is restricted to $\left[0, \frac{\pi}{2}\right]$ with a reflection on the $\left\{z=\frac{c_{3}}{2}\right\}$ plane. (3) The coordinate $\theta$ is restricted to $\left[0, \frac{\pi}{2}\right]$ with a rotation around the third axis.

The first stage of the algorithm consists to transform the spherical coordinates $(\theta, \phi)$ to the local chart. The output of this algorithm is the transformed coordinates $(\tilde{\theta}, \tilde{\phi})$, the permutation $\sigma$, and the set of signs s. This transformation must be seen as a composition of reflections and rotations of the hexahedron. Imagine a point inside of a hexahedron on the locus of the centroids represented in figure 4 . This point follows the transformation applied to the hexahedron. We would like to rotate the hexahedron in such a way that the point lies in the region close to the corner of the origin. This region corresponds approximately to the location of the local chart. To better understand this procedure, consider the example presented in figure 6 . This figure represents the global chart for $\chi=0.1, c_{1}=2, c_{2}=1.5$ and $c_{3}=1$. The spherical coordinates of the point $(\theta, \phi)$ denoted by 1 are located in the $\left[-\frac{\pi}{2}, 0\right] \times\left[\frac{\pi}{2}, \pi\right]$ region. The set of signs and the permutation are 
initialized to the identity $\mathrm{s}=\{1,1,1\}$ and $\sigma=\mathrm{id}$. To construct the point 2 , the coordinate $\phi$ of the point 1 is restricted to the interval $\left[0, \frac{\pi}{2}\right]$ with a reflection of the hexahedron on the $\left\{z=\frac{c_{3}}{2}\right\}$ plane. The set of signs becomes $\{1,1,-1\}$. The point 3 is constructed by a rotation of $\frac{\pi}{2}$ around the third axis. This rotation corresponds to a permutation of the first axis and the second axis and by reversing the direction on the first axis. Thus, the permutation becomes $\tau_{12}$ and the sign becomes $\{-1,1,-1\}$. Note that, at this point, figure 6 no longer represent the global map. The hexahedron has been rotated but the frame of spherical coordinates has not been changed. Thus, the representation in spherical coordinates should be redrawn by permuting the dimensions $c_{1}$ and $c_{2}$ of the hexahedron. The point 3 is now located in the local chart defined in figure 5 .

We propose the following 7 -step algorithm to transform the coordinates from the global chart to the local chart.

Step 1. Set $\tilde{\theta} \leftarrow \theta$ and $\tilde{\phi} \leftarrow \phi$.

Step 2. Translate $\tilde{\theta}$ and $\tilde{\phi}$ such that $(\tilde{\theta}, \tilde{\phi}) \in[-\pi, \pi] \times[0,2 \pi[$ by adding $\pm \pi$ as many times as necessary.

Step 3. Initialize $\mathrm{s} \leftarrow\{1,1,1\}$

Step 4. Crop $\tilde{\phi}$ to $\left[0, \frac{\pi}{2}\right]$ with the following instructions:

- If $\tilde{\phi} \in] \pi, 2 \pi\left[: \tilde{\phi} \leftarrow 2 \pi-\tilde{\phi}\right.$ and $\mathrm{s}_{3} \leftarrow-1$.

- If $\left.\tilde{\phi} \in] \frac{\pi}{2}, \pi\right]$, make a reflection on the third axis: $\tilde{\phi} \leftarrow \pi-\tilde{\phi}$ and $\mathrm{s}_{3} \leftarrow-\mathrm{s}_{3}$.

Step 5. Crop $\tilde{\theta}$ to $\left[0, \frac{\pi}{2}\right]$ with the following instructions:

- If $\left.\tilde{\theta} \in] \frac{\pi}{2}, \pi\right]$, rotate by $-\frac{\pi}{2}$ around the third axis: $\tilde{\theta} \leftarrow \tilde{\theta}-\frac{\pi}{2}$ and $\mathrm{s} \leftarrow\left\{1,-1, \mathrm{~s}_{3}\right\}$.

- If $\tilde{\theta} \in\left[-\pi,-\frac{\pi}{2}\left[\right.\right.$, rotate by $\pi$ around the third axis: $\tilde{\theta} \leftarrow \tilde{\theta}+\pi$ and $\mathrm{s} \leftarrow\left\{-1,-1, \mathrm{~s}_{3}\right\}$.

- If $\tilde{\theta} \in\left[-\frac{\pi}{2}, 0\left[\right.\right.$, rotate by $\frac{\pi}{2}$ around the third axis: $\tilde{\theta} \leftarrow \tilde{\theta}+\frac{\pi}{2}$ and $\mathrm{s} \leftarrow\left\{-1,1, \mathrm{~s}_{3}\right\}$.

Step 6. Set the permutation: If $\mathrm{s}_{1} \mathrm{~s}_{2}<0, \sigma \leftarrow \tau_{12}$ Else $\sigma \leftarrow$ id.

Step 7. Apply the permutation to the dimensions of the hexahedron $\left\{c_{1}, c_{2}, c_{3}\right\} \leftarrow \sigma \cdot\left\{c_{1}, c_{2}, c_{3}\right\}$.

At the end of this stage, we have determined $\sigma, \mathrm{s}$, and $(\tilde{\theta}, \tilde{\phi})$ which belong to the local chart.

\subsubsection{Stage 2: Compute the objective function and its gradient}

The second stage of the algorithm consists to evaluate the centroid and its partial derivatives in the local chart and to compute the gradient of the objective function. First, determine in which configuration Triangle, Penta, QuadEdge, QuadFace, or Hexa - the transformed coordinates belong to using the internal limits $\phi_{\star}^{\lim }(12)$ to $(16)$. Note that $\theta_{4}^{\mathrm{h}}$ can be placed before or after $\theta_{5}$ when $\chi>\frac{1}{6}$ depending on the

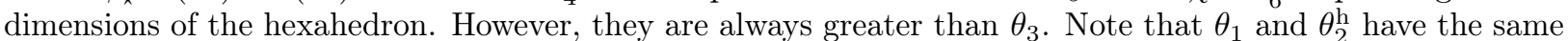
behavior but are always smaller than $\theta_{3}$. In particular, the sign of $\theta_{2}^{h}-\theta_{1}$ is the same as the sign of $\theta_{5}-\theta_{4}^{h}$. Once the configuration is determined, evaluate accordingly the centroid and the derivatives in the local chart using the formulas defined in section 3.2.2. Finally, compute the gradient of the objective function in the global chart using relation (25). For the implementation, we recommend to prefer any configuration over the Penta and Hexa configurations and to prefer the Penta configuration over the Hexa in the inequalities of the limit curves. Furthermore, we recommend to treat the case $\chi=\frac{1}{2}$ using only the limits of the Penta configuration which are the same as those of the Hexa configuration but are numerically more accurate.

Although it is not required by the algorithm, the centroid given in the local chart can be transformed to the global chart by applying the inverse of the permutation to its coordinates $\mathcal{C} \leftarrow \sigma^{-1} \cdot \mathcal{C}_{\text {loc }}$ and to the set of signs $\mathrm{s} \leftarrow \sigma^{-1} \circ \mathrm{s}$, and then by applying the correction $\mathcal{C}_{i} \leftarrow c_{i}-\mathcal{C}_{i}$ if $\mathrm{s}_{i}=-1$, where the $c_{i}$ correspond to the non-permuted dimensions of the cell. Here $\sigma^{-1}=\sigma$ since $\sigma=$ id or $\tau_{12}$.

At the end of this stage, we have determined $\mathcal{F}(\theta, \phi), \partial_{\theta} \mathcal{F}(\theta, \phi)$ and $\partial_{\phi} \mathcal{F}(\theta, \phi)$ which will be used in the optimization algorithm. 


\section{Numerical results}

\subsection{Introduction}

In this section, we demonstrate that the proposed analytic method outperforms the geometric approaches. Two criteria are evaluated: the runtime and the robustness. The former criterion is essential to reduce the wall clock time and total CPU time, especially for high performance computers while the latter is necessary to evaluate the consistency of the accuracy of the results.

Three geometric approaches are compared to our proposed method. The first, referred to as finite differences gradient, consists to reconstruct an approximation $\omega \in \mathfrak{A}(2)$ in the direction $\boldsymbol{n}$ using the flood algorithm proposed by Diot \& François [24]. The centroid of this approximation is computed using a formula for convex polyhedrons as presented in [25]. The partial derivatives of the centroid are evaluated with a centered finite-difference scheme which requires two more reconstructions per direction. The gradient of the objective function is then computed using formula (8). Overall, five calls to the flood algorithm are required to compute the objective function and its gradient. The second method, referred to as geometric gradient, consists to use only one call of the flood algorithm to compute the centroid and to evaluate the gradient by the method of Chen \& Zhang [17], which we have summarized using our notations in Appendix A. To compute the centroid of the reconstructed polyhedron, this second method requires to construct the vertex-face connectivities of the polyhedron at the term of the flood algorithm. The third method, referred to as optimized centroid, is an improvement of the second detailed in Appendix B.

In [24], the polyhedron is rotated such that the flood direction $\boldsymbol{n}$ corresponds to the axis $\boldsymbol{e}_{3}$. Two special cases must be treated if $\boldsymbol{n}= \pm \boldsymbol{e}_{3}$. When the partial derivatives are evaluated by finite differences near the poles, the singularity of the rotation creates some non-negligible perturbations in the values of the partial derivatives. Since the minimization algorithm relies on the accuracy of the derivatives, it is necessary to avoid the rotation. In Appendix B, we describe how to adapt the algorithm for any flood direction. This improvement is used in any geometric method tested in this paper.

All of these methods are implemented in the massively parallel open-source code Notus [26] which is dedicated to the modelization and simulation of incompressible fluid flows. Its numerical framework is the finite volume method on Cartesian staggered grids with a methodological focus on interfaces treatment (multi-material interface advection [18], surface tension computation [27], immersed boundary methods [28], etc.). The verification cases are conducted on a supercomputer with Intel Xeon E5-4640 processors and on a supercomputer with Intel Xeon Gold 6130 processors. The code was compiled with Intel Fortran Compiler 18.0. Although the runtime ratios given in this article are implementation-dependent and may vary with the architecture and the compilers, they are representative of the general behavior of the methods.

\subsection{Robustness and runtime ratios of the computation of the objective function and its gradient}

\subsubsection{Robustness}

To verify the proposed method, the centroid and the gradient computed by the formulas given in section 3 have been compared to those given by the finite differences gradient method and the geometric gradient method for a large number of configurations. The parameters varied are the cell dimensions $\left\{c_{1}, c_{2}, c_{3}\right\}$, the direction $\boldsymbol{n}$, and the volume fraction $\chi$. Although the position of the reference centroid $\mathcal{C}^{\star}$ does not matter, it is wise to select a position far away from the locus of the centroids $\mathfrak{L}^{\mathcal{V}}$ to avoid to nullify the term $\mathcal{C}^{\mathcal{V}}(\theta, \phi)-\mathcal{C}^{\star}$ in the gradient of the objective function which may hide potential errors in the partial derivatives of the centroid. The center of the hexahedron is a good choice for the reference centroid.

Regardless of the parameters, the distance between two centroids given by the two different methods is close to the machine precision. However, the gradient of the objective function does differ between the methods. As expected, the difference between the gradients given by finite differences and the proposed method is the highest. We found that the optimal value for the finite differences step is $\Delta \theta=\Delta \phi=10^{-9}$ that results in a difference of about $10^{-8}$ in magnitude. With the method of Chen \& Zhang [17], described in Appendix A, and the optimized centroid method discussed in Appendix B, this difference drops to the machine precision.

Although we verified our method on a large number of configurations, we have to challenge it on extreme cases. These include large aspect ratios of the hexahedron, small volume fractions, and the particular case 
$\chi=\frac{1}{2}$. Moreover, we have to verify our method when the interface passes through one or more vertices of the hexahedron which correspond to the case when the interface is between two or more configurations, that is when $\phi=\phi_{\star}^{\lim }(\theta)$. For each extreme case, the accuracy is measured by computing the $\ell_{\infty}$ norm of the difference between the values (objective function and gradient) given by the proposed method and the optimized centroid method on a large sampling of the local chart and the limit curves. Note that we took care to sample the end points of the limit curves which correspond to the cases where the interface passes through two, three, or four vertices of the hexahedron. Typically, we used 1000 samples per direction on the local chart and 1000 points per limit curve.

The results on these extreme cases show the importance of the inequalities used to determine the configuration on the local chart during the stage 2 of the algorithm (see 3.3.3). For numerical stability on the limit curves, we recommend to use any configuration over the Penta and Hexa configurations and to use the Penta configuration over the Hexa. For the cases with large aspect ratios, we have tested our method for various volume fractions on plate-like cells where two lengths are equal and the third one is 1000 times smaller and on needle-like cells where two lengths are equal and the third one is 1000 larger. Compared to the optimized centroid method, we found an error close to machine precision. For the case $\chi=\frac{1}{2}$, only the QuadFace and Hexa configurations remain. We found that the formulas of the limit curves of the Penta configurations are more numerically accurate that the formulas of the limit curves of the Hexa configuration although they are the same analytically. For small volume fractions $\chi \rightarrow 0$, the difference between our method and the geometric method is very close until $\chi \approx 10^{-10}$. For smaller $\chi$, the difference increases for the gradient but it is difficult to tell which method gives the right result.

\subsubsection{Runtime ratios}

To determine the performances of the various methods, the evaluation of the objective function and its gradient are computed for a large number of configurations. The ratio between the runtime of the methods and the runtime of our proposed method is then calculated. To generate the different cases, the direction $\boldsymbol{n}$ and the volume fraction $\chi$ are evenly sampled. We found that the dimensions of the cell do not change the runtime ratios.

We use an exponential sampling for the volume fraction $\chi$ in the range $10^{-10}$ to $\frac{1}{2}$ which means that $\log (\chi)$ is uniform in $\left[\log \left(10^{-10}\right), \log \left(\frac{1}{2}\right)\right]$. To sample the direction $\boldsymbol{n}$, we cannot use a linear sampling on $\theta$ and $\phi$ as it does not evenly distribute the points on the unit sphere since the poles are more densely sampled than the equator. Instead, the points on the sphere are generated along a spherical Fibonacci grid [29] which gives a good approximation of an evenly distribution of points on the sphere. The coordinates of these points are given in equation (26) for $2 N+1$ samples.

$$
\forall k \in \llbracket-N, N \rrbracket \quad \theta_{k}=2 \pi \frac{\bmod (k, \varphi)}{\varphi} \quad \phi_{k}=\arccos \left(\frac{2 k}{2 N+1}\right) \quad \text { where } \varphi=\frac{1+\sqrt{5}}{2}
$$

The results are presented in table 1 for 1000 samples of $\chi$ and 25001 samples of $\boldsymbol{n}$ which makes a total of 25001000 cases. Increasing the number of samples does not significantly change the runtime ratios.

\begin{tabular}{lr}
\hline Method & Runtime ratio \\
\hline Finite differences gradient & 237 \\
Geometric gradient (Appendix A) & 63 \\
Optimized centroid (Appendix B) & 43 \\
Analytic reconstruction & $\mathbf{1}$ \\
\hline
\end{tabular}

Table 1: Runtime ratios between geometric methods and analytic method for the computation of the objective function and its gradient.

From table 1, we observe that the geometric gradient method is almost 4 times faster than the finite differences gradient method. This result is expected since it replaces four calls to the flood algorithm with simple a calculation on a polygon (see Appendix A). The optimized centroid method that is proposed 
in Appendix B is 1.5 times faster than the geometric gradient method. This gain is obtained by computing the centroid directly during the flood algorithm instead of reconstructing a polyhedron structure to subsequently compute its centroid. Also, using the method of Chen \& Zhang [17] to compute the gradient makes the optimized centroid method 5.5 faster than the finite differences gradient method. Drastic increases in performance is further obtained through our proposed analytic method which is 237 times faster than the finite differences method and 43 times faster than the most competitive geometric method. These results show that the proposed method should be used to improve the numerical simulation runtime.

\subsection{Robustness and runtime ratios for reconstructions with a minimization algorithm}

Although we verified our method on the computation of the objective function and its gradient, we also tested the behavior of the various methods coupled with a minimization algorithm to further analyze their behavior. In this article, we chose the BFGS algorithm along with the line-search algorithm described in [30]. We use the classic initial guess based on the centroid of the cell $\mathcal{C}(\Omega)$ and the reference centroid $\mathcal{C}^{\star}$ defined in the following equation:

$$
\boldsymbol{n}\left(\theta_{0}, \phi_{0}\right)=\frac{\mathcal{C}^{\star}-\mathcal{C}(\Omega)}{\left|\mathcal{C}^{\star}-\mathcal{C}(\Omega)\right|}
$$

The objective function (7) contains several local minima and sometimes several global minima, such as the case where the reference centroid is at the center of the cell. We will exploit this property to measure the robustness of our proposed method compared to the others since the less robust methods are more likely to make the minimization algorithm fall into local minima.

For the line-search algorithm proposed in [30] we use the currently optimized parameters: $\rho=0.25$, $\sigma=0.5, \tau_{1}=3, \tau_{2}=0.1$ and $\tau_{3}=0.5$. The minimization algorithm is the same for all the tested methods and stops when it reaches a maximum number of iterations or the norm of the gradient of the objective function falls below a prescribed tolerance value.

We have conducted the robustness study on two sets of one million random-generated cases. In both sets, the dimensions of the cell for each case are computed with the formula $c_{i}=\alpha_{i} \cdot 10^{\beta}$ where $\alpha_{i} \in[0.1,1[$ and $\beta \in[-3,3]$ are randomly-generated with a uniform distribution. The volume fractions $\chi \in\left[10^{-7}, \frac{1}{2}\right]$ are generated with a exponential distribution. The two sets differ by the way the reference centroids are generated. In the first set, the reference centroids lie on the locus of the centroids. That means that the minimum value of the objective function (7) is zero. This set will be referred to as the exact reconstruction cases. To generate this set, a direction $\boldsymbol{n}$ is randomly generated with a uniform distribution and a flood algorithm is used to compute the reference centroids. In the second set, the reference centroids are randomly generated over the cell with a uniform distribution. This set will be referred to as the random reconstruction cases. The tolerance value of the BFGS algorithm - the norm of the gradient of the objective function - is set to $10^{-14}$ and the maximum number of iterations is set to 400 . These parameters can be relaxed for a practical use of MOF, but here, we want to detect whether one of the methods prevents to reach a small residual.

\subsubsection{Robustness on exact reconstructions with a BFGS algorithm}

On the exact reconstruction cases, the four methods - finite differences gradient, geometric gradient, optimized centroid, and analytical reconstruction — give the same statistical results. Note that each iteration of the BFGS algorithm requires one evaluation of the gradient and several iterations of the line-search algorithm. Each iteration of the line-search algorithm requires one evaluation of the gradient and at least one more for the bracketing phase. The median of the number of gradient evaluations in the BFGS is 11 , the median of the number of gradient evaluations in the line-search algorithm is 53 and the median of the sum of gradient evaluations in the BFGS and line-search is 62 .

To understand the similarity of the statistics, we have evaluated the quality of the computed minimum for each pair of methods by comparing the final values of the objective function. With this criterion, we are not able to differentiate the methods since they give the same results with no apparent differences. We explain this behavior by remarking that the term $\mathcal{C}^{\mathcal{V}}(\theta, \phi)-\mathcal{C}^{\star}$ in the gradient vanishes when the BFGS gets closer to the minimum and, as a result, the numerical errors on the partial derivatives are reduced by this 
term. Furthermore, the initial guess makes this term quite small from the initial step of the algorithm. For the random reconstruction cases, this term does not vanish and we are able to see the differences.

\subsubsection{Robustness of the random reconstructions with a BFGS algorithm}

On the random reconstruction cases, the behavior of the BFGS differs depending on the method used. Table 2 presents the median of the number of gradient evaluations in the BFGS, the line-search, and the sum of the BFGS and the line-search algorithms.

\begin{tabular}{lccc}
\hline Method & BFGS & line-search & BFGS + line-search \\
\hline Finite differences gradient & 19 & 167 & 188 \\
Geometric gradient (Appendix A) & 20 & 160 & 181 \\
Optimized centroid (Appendix B) & 19 & 152 & 171 \\
Analytic reconstruction & $\mathbf{1 8}$ & $\mathbf{1 5 0}$ & $\mathbf{1 6 8}$ \\
\hline
\end{tabular}

Table 2: Median of the number of calls of the gradient in the different parts of the minimization algorithm and for the different reconstruction methods.

This time, the term $\mathcal{C}^{\mathcal{V}}(\theta, \phi)-\mathcal{C}^{\star}$ does not vanish in the gradient of the objective function and the errors in the approximation of the partial derivatives deteriorates the convergence of the BFGS algorithm. The exponential sampling guarantees an uniform distribution of $\log (\chi)$ in the range $\left[\log \left(10^{-7}\right), \log \left(\frac{1}{2}\right)\right]$. This implies that a lot of cases have small volume fractions for which the objective function if very flat with sudden and abrupt variations. As a consequence, more iterations of the minimization algorithm are required to reach its convergence criteria which explains the large values obtained for the median number of calls of the gradient. We observe that the analytic methods requires less calls to the gradient compared to other methods. We explain this result by the smoothness of the analytic formulas compared to the geometric methods. However, we note that the results of the optimized centroid method are close to the results of the proposed method.

\subsubsection{Runtime ratios for reconstructions with a BFGS algorithm}

For these exact and random sets of cases, the runtime ratios between the various methods and the proposed method have been computed and summed up in table 3.

\begin{tabular}{lcc}
\hline \multirow{2}{*}{ Method } & \multicolumn{2}{c}{ Runtime ratio } \\
& exact & random \\
\hline Finite differences gradient & 104 & 193 \\
Geometric gradient (Appendix A) & 28 & 51 \\
Optimized centroid (Appendix B) & 19 & 28 \\
Analytic reconstruction & $\mathbf{1}$ & $\mathbf{1}$ \\
\hline
\end{tabular}

Table 3: Runtime ratios between geometric methods and analytic method using the BFGS algorithm.

These results differs from table 1 since the runtime cumulates of the evaluation of the gradient and the BFGS itself. Anyway, we observe that the analytic reconstruction method still outperforms the geometric methods.

\subsection{Reconstruction of a face-centered cubic arrangement of spheres}

To complete the set of numerical tests, we compared the behavior of the various methods on two more actual cases composed of a face-centered cubic (FCC) arrangement of spheres in a unit cube. The first case presented in the left of figure 7 represents the elemental chunk of a FCC arrangement of 14 spheres. This case was performed in a randomly perturbed rectilinear grid composed of 64 cells per direction. The volume 
and the centroid of each cells have been initialized using a sampling method and the polyhedrons have been reconstructed with the $\mathrm{MOF}$ method using the analytic centroid method.
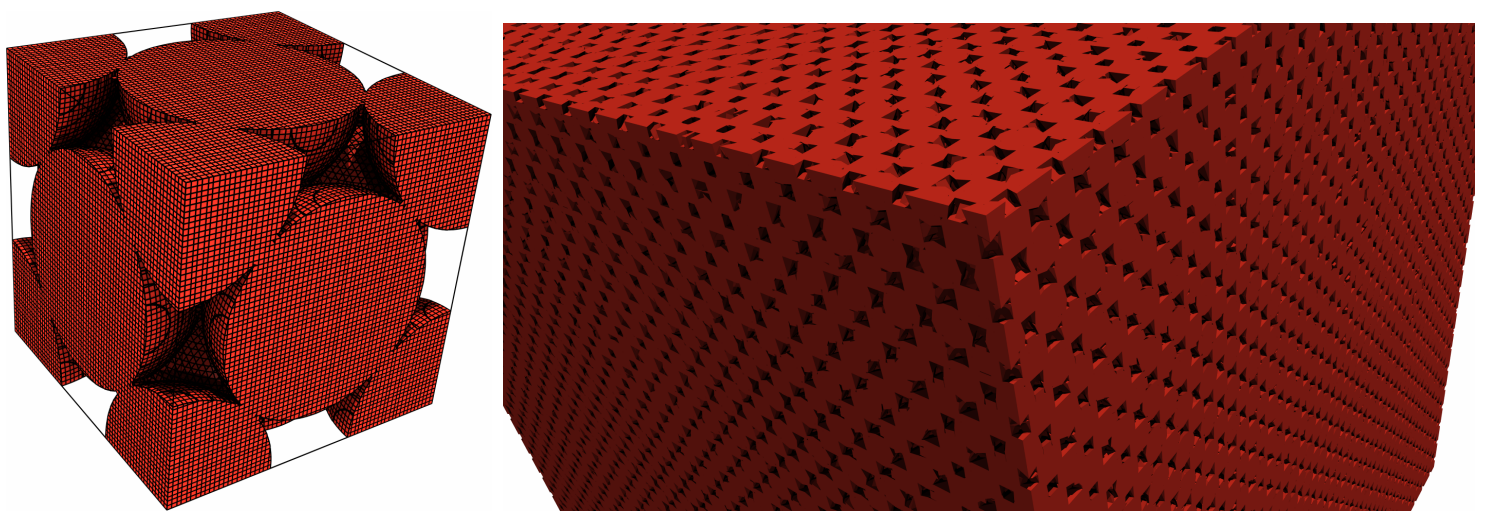

Figure 7: Face-centered cubic arrangement of spheres reconstructed using the analytic centroid method. Left: one chunk of the face-centered cubic arrangement reconstructed on a perturbed $64^{3}$ rectilinear grid. Right: $26^{3}$ chunks loosely initialized and tiled in a perturbed $128^{3}$ rectilinear grid.

Measuring the runtime ratios of the various methods requires a large computation for the results to be considered as statistically converged. To increase the number of reconstructions, we present a second case where the chunk was shrunk and tiled 26 times per direction in the unit cube. The volumes and the centroids have been initialized with a coarse sampling in each cells. The randomly perturbed rectilinear grid was generated starting from a regular rectilinear grid composed of 128 cells per direction. In each directions, the position of each faces are randomly shifted by a step $\alpha h_{0}$ where $h_{0}=\frac{1}{128}$ is the original space step and $\alpha$ is a random number in the range $[-0.2,0.2]$. This configuration contains 74439 spheres and required 3354272 calls to the BFGS algorithm to be reconstructed. The right of figure 7 represents a close-up view of the polyhedrons reconstructed by the MOF method for this second test. The runtime measures were conducted on a single CPU to increase the accuracy of the ratio and are presented in table 4 . To give an idea of the time saved, this case with our analytic method ran in $273 \mathrm{~s}$ and in $24485 \mathrm{~s}$ with the finite differences gradient method.

\begin{tabular}{lr}
\hline Method & Runtime ratio \\
\hline Finite differences gradient & 89 \\
Geometric gradient (Appendix A) & 22 \\
Optimized centroid (Appendix B) & 17 \\
Analytic reconstruction & $\mathbf{1}$ \\
\hline
\end{tabular}

Table 4: Runtime ratios between geometric methods and analytic method for the reconstruction of a face-centered cubic arrangement of spheres in a $128^{3}$ randomly perturbed rectilinear grid.

This time, the runtime cumulates the evaluation of the gradients, the minimization algorithm and the whole MOF algorithm on all the cells. As before, we observe that the analytic reconstruction method still performs better than the other methods.

To conclude, all the methods except the finite differences gradient method offers approximately the same accuracy. This is due to the absence of approximations in the evaluation of the partial derivatives of the objective function. These methods can be differentiated by their runtime and, as shown in tables 1,3 , and 4 , our analytic reconstruction method outperforms the other methods. 


\section{Conclusion}

In this article we have developed a new fast and robust approach to solve the minimization problem of the moment-of-fluid method in 3D on rectangular hexahedrons. The key idea is to find a global parametrization in spherical coordinates of the locus of the centroids at fixed volume. This allows to compute the objective function and its gradient with fully analytical formulas which avoid the use of a computationally intensive geometric flood algorithm. The numerical results show that our analytical method is up to 200 times faster than the geometric approaches. We have also adapted and improved the flood algorithm [24] for the moment-of-fluid method for general convex cells. In future work, we will extend our methodology of the centroid locus parametrization to other cell shapes, for instance, in $2 \mathrm{D}$ on convex polygons or in $3 \mathrm{D}$ on tetrahedrons.

\section{Appendix A. Analytic gradient from the geometric approach}

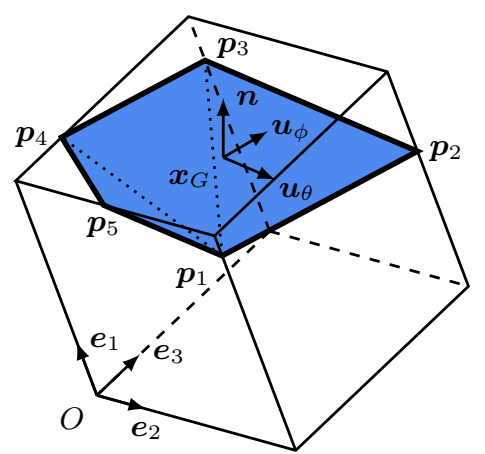

Figure A.8: Example of a top polygon (Penta configuration, $\chi=0.9$ ). The points $\boldsymbol{p}_{i}$ denote the vertices of the polygon and $\boldsymbol{x}_{G}$ denotes the centroid of the top polygon. The Cartesian frame and the spherical frame are represented in there respective positions.

In this section, we present the formulas to compute the gradient of the objective function proposed by Chen \& Zhang [17]. The input data of this algorithm is the surface of the intersection of a half-space with the polyhedron given by the flood algorithm. This surface will be denoted by $\mathfrak{P}$ and will be referred to as top polygon. Figure A.8 gives an example of a top polygon in the Penta configuration. The top polygon is composed of $n$ vertices denoted by $\boldsymbol{p}_{i}$ for $i \in \llbracket 1, n \rrbracket$. The algorithm to compute the gradient of the objective function can be decomposed in four steps.

Step 1. Compute the surface $S$ and the centroid $\boldsymbol{x}_{G}$ of the top polygon $\mathfrak{P}$ in the Cartesian frame $\left(O ; \boldsymbol{e}_{1}, \boldsymbol{e}_{2}, \boldsymbol{e}_{3}\right)$ with equations (A.1) and (A.2).

$$
\begin{gathered}
S=\frac{1}{2} \sum_{i=2}^{n-1}\left|\left(\boldsymbol{p}_{i}-\boldsymbol{p}_{1}\right) \times\left(\boldsymbol{p}_{i+1}-\boldsymbol{p}_{1}\right)\right| \\
\boldsymbol{x}_{G}=\boldsymbol{p}_{1}+\frac{1}{6 S} \sum_{i=2}^{n-1}\left|\left(\boldsymbol{p}_{i}-\boldsymbol{p}_{1}\right) \times\left(\boldsymbol{p}_{i+1}-\boldsymbol{p}_{1}\right)\right|\left(\left(\boldsymbol{p}_{i}-\boldsymbol{p}_{1}\right)+\left(\boldsymbol{p}_{i+1}-\boldsymbol{p}_{1}\right)\right)
\end{gathered}
$$

Step 2. Transform the points into the spherical frame $\left(\boldsymbol{x}_{G} ; \boldsymbol{u}_{\theta}, \boldsymbol{u}_{\phi}, \boldsymbol{n}\right)$ where the origin is set to the centroid of the top polygon. The unit vectors of the spherical basis can be expressed in the Cartesian basis with equation (A.3).

$$
\begin{aligned}
\boldsymbol{u}_{\theta} & =-\sin (\theta) \boldsymbol{e}_{1}+\cos (\theta) \boldsymbol{e}_{2} \\
\boldsymbol{u}_{\phi} & =\cos (\theta) \cos (\phi) \boldsymbol{e}_{1}+\sin (\theta) \cos (\phi) \boldsymbol{e}_{2}-\sin (\phi) \boldsymbol{e}_{3} \\
\boldsymbol{n} & =\cos (\theta) \sin (\phi) \boldsymbol{e}_{1}+\sin (\theta) \sin (\phi) \boldsymbol{e}_{2}+\cos (\phi) \boldsymbol{e}_{3}
\end{aligned}
$$


Any point $\boldsymbol{p}$ in the Cartesian frame can be transformed into a point $\tilde{\boldsymbol{p}}$ in the spherical frame by the relation (A.4).

$$
\tilde{\boldsymbol{p}}=\left[\begin{array}{c}
\left(\boldsymbol{p}-\boldsymbol{x}_{G}\right) \cdot \boldsymbol{u}_{\theta} \\
\left(\boldsymbol{p}-\boldsymbol{x}_{G}\right) \cdot \boldsymbol{u}_{\phi} \\
\left(\boldsymbol{p}-\boldsymbol{x}_{G}\right) \cdot \boldsymbol{n}
\end{array}\right]
$$

Step 3. Calculate three quadratic integrals over the top polygon. Consider any polynomial function $\varphi: \mathbb{R}^{3} \rightarrow \mathbb{R}$ of degree at most two. The integral of $\varphi$ over the top polygon can be exactly computed using the sum of a 3-point Gauss quadrature, as defined in formula (A.5). This formula comes from the decomposition of the polygon into triangles as represented by dotted lines in figure A.8. Note that the points must be transformed into the spherical frame using the previous relation (A.4).

$$
I_{\varphi}=\frac{1}{6} \sum_{i=2}^{n-1}\left|\left(\tilde{\boldsymbol{p}}_{i}-\tilde{\boldsymbol{p}}_{1}\right) \times\left(\tilde{\boldsymbol{p}}_{i+1}-\tilde{\boldsymbol{p}}_{1}\right)\right|\left(\varphi\left(\frac{\tilde{\boldsymbol{p}}_{i}+\tilde{\boldsymbol{p}}_{1}}{2}\right)+\varphi\left(\frac{\tilde{\boldsymbol{p}}_{i}+\tilde{\boldsymbol{p}}_{i+1}}{2}\right)+\varphi\left(\frac{\tilde{\boldsymbol{p}}_{i+1}+\tilde{\boldsymbol{p}}_{1}}{2}\right)\right)
$$

Compute the quantities $I_{x x}, I_{x y}$ and $I_{y y}$ which correspond, respectively, to $\varphi=x^{2}, \varphi=x y$ and $\varphi=y^{2}$. In these formulas, $x, y$ and $z$ are the coordinates of any point in the spherical frame given by $x \boldsymbol{u}_{\theta}+y \boldsymbol{u}_{\phi}+z \boldsymbol{n}$.

Step 4. Finally, compute the partial derivatives in the spherical frame and the gradient of the objective function. The partial derivatives in the spherical frame are computed using relation (A.6).

$$
\begin{aligned}
& \partial_{\theta} \widetilde{\mathcal{C V}^{\mathcal{V}}}(\theta, \phi)=-\frac{\sin (\phi)}{\mathcal{V}}\left[I_{x x}, I_{x y}, 0\right] \\
& \partial_{\phi} \widetilde{\mathcal{C}^{\mathcal{V}}}(\theta, \phi)=-\frac{1}{\mathcal{V}}\left[I_{x y}, I_{y y}, 0\right]
\end{aligned}
$$

The gradient of the objective function is computed with formula (A.7). Note that the centroid and the reference centroid must be transformed into the spherical frame using relation (A.4).

$$
\begin{aligned}
& \partial_{\theta} \mathcal{F}(\theta, \phi)=2\left(\widetilde{\mathcal{C}^{\mathcal{V}}}(\theta, \phi)-\widetilde{\mathcal{C}}^{\star}\right) \cdot \partial_{\theta} \widetilde{\mathcal{C}^{\mathcal{V}}}(\theta, \phi) \\
& \partial_{\phi} \mathcal{F}(\theta, \phi)=2\left(\widetilde{\mathcal{C}^{\mathcal{V}}}(\theta, \phi)-\widetilde{\mathcal{C}}^{\star}\right) \cdot \partial_{\phi} \widetilde{\mathcal{C}^{\mathcal{V}}}(\theta, \phi)
\end{aligned}
$$

\section{Appendix B. Improvements of the flood algorithm for the moment-of-fluid method}

In this section, we present some optimizations related to the flood algorithm proposed by Diot \& François [24]. The first optimization consists in getting rid of the rotation of the polyhedron in the reference frame since it induces some singularities in the derivative when $\boldsymbol{n}= \pm \boldsymbol{e}_{3}$. The second optimization consists in evaluating the centroid during the reconstruction.
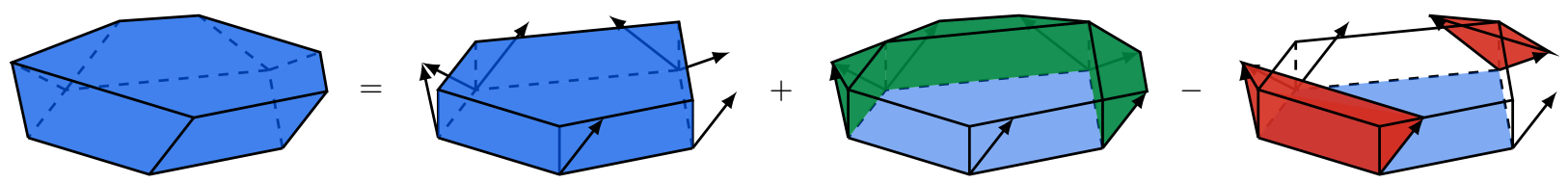

Figure B.9: Decomposition of a prismatoid in the sum of three terms. First, add a right prism. Second, add some tetrahedron and triangular prisms (in green). Third, subtract some tetrahedron and triangular prisms (in red). The arrows denote the unit tangent of the edges of the prismatoid.

The method proposed by Diot \& François consists in bracketing the position of the interface between two parallel slices of the polyhedron. Each slice corresponds to a plane that passes through, at least, one vertex of the polyhedron. The slices are sorted in such a way that the distances of the planes on the flood axis $\boldsymbol{n}$ are in increasing order. The shape between two consecutive planes is a polyhedron called a prismatoid. All the 
vertices of a prismatoid are contained in one or the other plane as show by the example in figure B.9. The flood algorithm consists in computing the volume of the prismatoids one after the other until the reference volume is exceeded. Once the reference volume exceeded, the position of the interface is found inside the last prismatoid (refer to [24] for the method). Here, we propose an alternative method to compute the volume of the prismatoids for any direction $\boldsymbol{n}$ instead of $\boldsymbol{n}=\boldsymbol{e}_{3}$ in the original article. Then we present new formulas to evaluate their centroids.

In the article of Diot \& François, the volume of a prismatoid is computed as the volume of a right prism plus the volume of some tetrahedrons and triangular prisms minus the volume of some tetrahedrons and triangular prisms (refer to figure B.9). In figure B.10, we present the notations of the known quantities during the computation of the volume. The base of the right prism is composed of $n$ vertices $\left(\boldsymbol{p}_{i}\right)_{i \in \llbracket 1, n \rrbracket}$ that forms the bottom plane of the prismatoid and its height is denoted by $h$. The tetrahedron is generated by three unit vectors $\boldsymbol{u}, \boldsymbol{v}$ and $\boldsymbol{n}$. The triangular prism is generated by the flood direction $\boldsymbol{n}$ and the normal of one of its face $\boldsymbol{n}_{f}$.

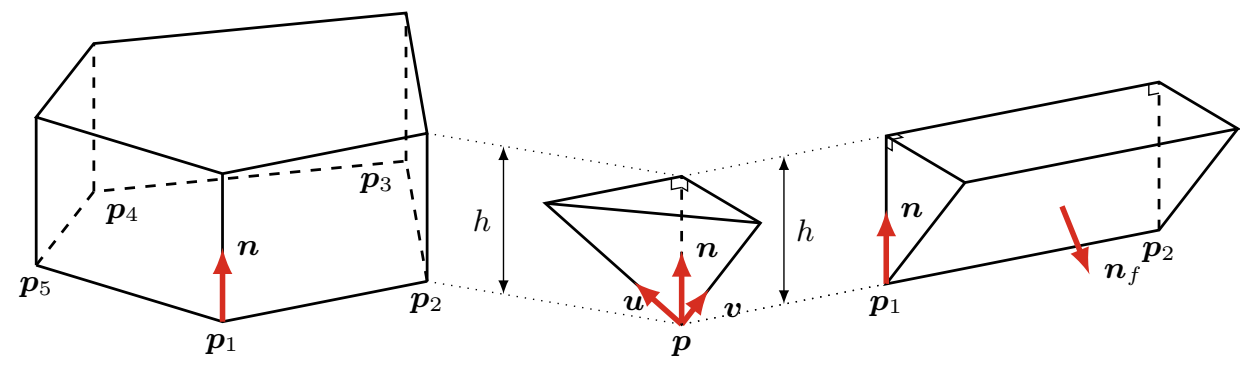

Figure B.10: Decomposition of the prismatoid. Left: right prism. Middle: tetrahedron. Right: triangular prism.

The volume of a tetrahedron and its centroid can be computed using equation (B.1).

$$
\mathcal{V}_{\text {tetra }}=\frac{h^{3}}{6}\left(\frac{\boldsymbol{u}}{\boldsymbol{u} \cdot \boldsymbol{n}} \times \frac{\boldsymbol{v}}{\boldsymbol{v} \cdot \boldsymbol{n}}\right) \cdot \boldsymbol{n} \quad \mathcal{C}_{\text {tetra }}=\boldsymbol{p}+\frac{h}{4}\left(\boldsymbol{n}+\frac{\boldsymbol{u}}{\boldsymbol{u} \cdot \boldsymbol{n}}+\frac{\boldsymbol{v}}{\boldsymbol{v} \cdot \boldsymbol{n}}\right)
$$

The volume of a triangular prism and its centroid can be computed using equation (B.2).

$$
\mathcal{V}_{\mathrm{tri}}=-\frac{h^{2}}{2}\left(\left|\boldsymbol{p}_{2}-\boldsymbol{p}_{1}\right| \frac{\boldsymbol{n} \cdot \boldsymbol{n}_{f}}{\left|\boldsymbol{n} \times \boldsymbol{n}_{f}\right|}\right) \quad \mathcal{C}_{\mathrm{tri}}=\frac{\boldsymbol{p}_{1}+\boldsymbol{p}_{2}}{2}+\frac{h}{3}\left(\frac{\boldsymbol{n}_{f} \times\left(\boldsymbol{p}_{2}-\boldsymbol{p}_{1}\right)}{\left|\boldsymbol{n} \times \boldsymbol{n}_{f}\right|\left|\boldsymbol{p}_{2}-\boldsymbol{p}_{1}\right|}\right)
$$

The volume of a right prism and its centroid can be computed using equations (B.3a) and (B.3b).

$$
\begin{gathered}
S=\frac{1}{2} \sum_{i=2}^{n-1}\left|\left(\boldsymbol{p}_{i}-\boldsymbol{p}_{1}\right) \times\left(\boldsymbol{p}_{i+1}-\boldsymbol{p}_{1}\right)\right| \\
\mathcal{V}_{\text {prism }}=h S \quad \mathcal{C}_{\text {prism }}=\frac{h}{2} \boldsymbol{n}+\boldsymbol{p}_{1}+\frac{1}{6 S} \sum_{i=2}^{n-1}\left|\left(\boldsymbol{p}_{i}-\boldsymbol{p}_{1}\right) \times\left(\boldsymbol{p}_{i+1}-\boldsymbol{p}_{1}\right)\right|\left(\left(\boldsymbol{p}_{i}-\boldsymbol{p}_{1}\right)+\left(\boldsymbol{p}_{i+1}-\boldsymbol{p}_{1}\right)\right)
\end{gathered}
$$

The centroid of a prismatoid and the centroid of the final polyhedron are computed using the additivity of the first momentum, that is, using the pseudo-formula (B.4).

$$
\mathcal{C}=\frac{\sum \mathcal{V}_{\text {tetra }} \mathcal{C}_{\text {tetra }}+\sum \mathcal{V}_{\text {tri }} \mathcal{C}_{\text {tri }}+\sum \mathcal{V}_{\text {prism }} \mathcal{C}_{\text {prism }}}{\sum \mathcal{V}_{\text {tetra }}+\sum \mathcal{V}_{\text {tri }}+\sum \mathcal{V}_{\text {prism }}}
$$

Remark that it is more efficient to write the algorithm in terms of first momentum instead of centroid. The centroid of the final polyhedron can be easily computed by dividing its first momentum by the total volume which is equal to the reference volume. 


\section{Appendix C. Proof of the formulas for the parametrization of the locus of the centroids}

In this appendix, we give a proof of the formulas given in section 3. For each configuration - Triangle, QuadEdge, QuadFace, Penta, and Hexa - the same methodology is used to obtain the analytic formulas. First, we remark that the surface of the half-space intersects between three and six edges of the hexahedron. In each configuration, we denote $A, B$, and $C$ three of these intersection points. The other points, when they exist, can be deduced from the coordinates of these points since they belong to the same plane. We define $\alpha$, $\beta$, and $\gamma$ the coordinates of the points $A, B$, and $C$ on the respective directions of their edges. The volume and the centroid of the intersection of the half-space and the rectangular hexahedron is then computed by a tetrahedral decomposition and by using the additivity of the volume and the first momentum. We recall that the centroid of a tetrahedron is equal to the centroid of its vertices. Then, some inequalities between $\alpha, \beta$, and $\gamma$ are established from the geometric constraints of the different configurations. Next, the volume $\mathcal{V}$ is imposed to give a relation $\beta=\beta(\alpha, \gamma, \mathcal{V})$. At this step, the centroid is described locally as a function of $\alpha$ and $\gamma$ for a fixed volume $\mathcal{V}$. The definition domain $\mathcal{D}_{\star}^{\mathcal{V}}$ of the local parametrization and its limit curves in $(\alpha, \gamma)$ are given for each configuration. The concatenation of these domains is illustrated in figure C.11 for $\chi \leq \frac{1}{6}$ and $\chi>\frac{1}{6}$. These domains are mapped in the $(\theta, \phi)$ chart (figure 5) and the domain enclosed by the bold lines corresponds to the left configurations. Finally, we give the parametrizations and the associated limit curves in spherical coordinates $(\theta, \phi)$ defined in section 3.

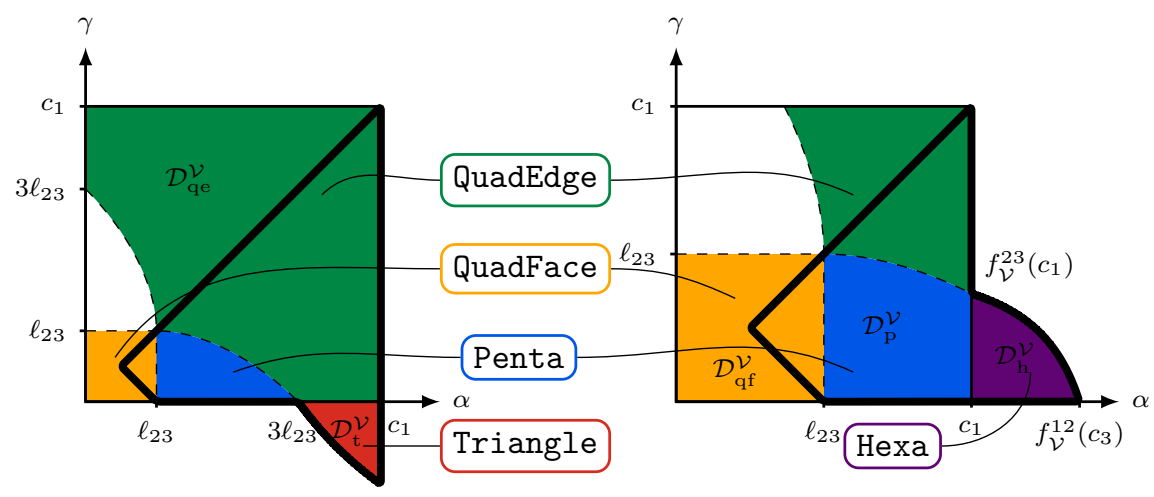

Figure C.11: Local charts in $(\alpha, \gamma)$ of all the configurations. The bold region corresponds to the left configurations of the local chart in $(\theta, \phi)$. (left) $\chi \leq \frac{1}{6}$. (right) $\chi>\frac{1}{6}$.

\section{Appendix C.1. Definitions and notations}

In the same way as for equation (6), the normal to the plane is defined as:

$$
\boldsymbol{n}=\frac{1}{\sqrt{a_{1}^{2}+a_{2}^{2}+a_{3}^{2}}}\left[\begin{array}{l}
a_{1} \\
a_{2} \\
a_{3}
\end{array}\right]=\left[\begin{array}{c}
\sin (\phi) \cos (\theta) \\
\sin (\phi) \sin (\theta) \\
\cos (\phi)
\end{array}\right]
$$

where $\theta \in[-\pi, \pi]$ and $\phi \in[0, \pi]$ are the spherical coordinates. The interface $\mathfrak{P}$ defined in (10) can be alternatively defined by:

$$
\mathfrak{P}=\left\{(x, y, z) \in \mathbb{R}^{3} \mid a_{1} x+a_{2} y+a_{3} z=1\right\}
$$

The coefficients $a_{1}, a_{2}$, and $a_{3}$ will be referred to as coefficients of the plane. We restrict $\theta$ to $[0, \pi / 2]$ and $\phi$ to $[0, \pi / 2]$ by symmetry, hence, $\cos (\phi), \cos (\theta), \tan (\phi), \tan (\theta), a_{1}, a_{2}$, and $a_{3}$ are positive. From (C.1), we have the relations:

$$
\frac{a_{2}}{a_{1}}=\tan (\theta) \quad \frac{a_{3}}{a_{1}}=\cot (\phi) \sec (\theta)
$$

In this appendix, we will provide the details of the parametrizations of the limit curves and centroids for the Left region. The equation (C.3) will give the two following important quantities involved in the Left 
region: $T_{2}^{l}=c_{2} \tan (\theta)$ and $T_{3}^{l}=c_{3} \cot (\phi) \sec (\theta)$. The parametrizations associated to the Right and Bottom regions can be easy deduced from the Left region with a circular permutation of the axes. For the Right region the permutation $x \rightarrow y \rightarrow z \rightarrow x$ is applied and leads to

$$
\frac{a_{2}^{r}}{a_{1}^{r}}=\frac{a_{3}}{a_{2}}=\cot (\phi) \csc (\theta) \quad \frac{a_{3}^{r}}{a_{1}^{r}}=\frac{a_{1}}{a_{2}}=\cot (\theta)
$$

which correspond to the two following important quantities involved in the Right region: $T_{2}^{r}=c_{3} \cot (\phi) \csc (\theta)$ and $T_{3}^{r}=c_{1} \cot (\theta)$. For the remaining Bottom region the permutation $x \rightarrow z \rightarrow y \rightarrow x$ is applied and leads to

$$
\frac{a_{2}^{b}}{a_{1}^{b}}=\frac{a_{1}}{a_{3}}=\tan (\phi) \cos (\theta) \quad \frac{a_{3}^{b}}{a_{1}^{b}}=\frac{a_{2}}{a_{3}}=\tan (\phi) \sin (\theta)
$$

that correspond to the two following important quantities involved in the Bottom region: $T_{2}^{b}=c_{1} \tan (\phi) \cos (\theta)$ and $T_{3}^{b}=c_{2} \tan (\phi) \sin (\theta)$. The parametrizations in the Right (respectively Bottom) region are then computed by replacing $T_{i}^{l}$ by $T_{i}^{r}$ (respectively $T_{i}^{b}$ ) and by applying a circular permutation of the coordinates as in (14) to (15) and (18) to (21). We will now detail how to obtain limit curves and centroids in the Left region.

We consider a rectangular hexahedron $\Omega=\left[0, c_{1}\right] \times\left[0, c_{2}\right] \times\left[0, c_{3}\right]$. We recall that the reference volume is denoted by $\mathcal{V}$ and the associated volume fraction $\frac{\mathcal{V}}{c_{1} c_{2} c_{3}}$ is denoted by $\chi$. We make the assumption that $\chi \leq \frac{1}{2}$ since the case $\chi>\frac{1}{2}$ can be treated by considering the complementary problem. In this appendix, we use the following notations $(i, j \in\{1,2,3\})$ already defined in (11):

$$
\ell_{i j}=\frac{2 \mathcal{V}}{c_{i} c_{j}} \quad T_{3}^{r}=c_{1} \cot (\theta) \quad T_{2}^{l}=c_{2} \tan (\theta) \quad T_{3}^{l}=c_{3} \cot (\phi) \sec (\theta)
$$

The following functions will be used in the definition of the limit curves of the local charts. The roots of the following second degree polynomial in $\gamma$ given by $\gamma^{2}+\alpha \gamma+\alpha^{2}-3 \ell_{i j} \alpha=\left(\gamma-f_{\mathcal{V}}^{i j}(\alpha)\right)\left(\gamma-\tilde{f}_{\mathcal{V}}^{i j}(\alpha)\right)$ are equal to:

$$
f_{\mathcal{V}}^{i j}(x)=\frac{1}{2}\left(-x+\sqrt{12 \ell_{i j} x-3 x^{2}}\right) \quad \tilde{f}_{\mathcal{V}}^{i j}(x)=\frac{1}{2}\left(-x-\sqrt{12 \ell_{i j} x-3 x^{2}}\right)
$$

The function $f_{\mathcal{V}}^{i j}$ is well defined and positive on $\left[0,3 \ell_{i j}\right]$, monotonically increasing on $\left[0, \ell_{i j}\right]$ and monotonically decreasing on $\left[\ell_{i j}, 3 \ell_{i j}\right]$. It verifies the identities $f_{\mathcal{V}}^{i j}(0)=0, f_{\mathcal{V}}^{i j}\left(\ell_{i j}\right)=\ell_{i j}$, and $f_{\mathcal{V}}^{i j}\left(3 \ell_{i j}\right)=0$. We also introduce $g_{\mathcal{V}}$ as:

$g_{\mathcal{V}}(\alpha)=c_{1} \frac{2 \alpha^{2}+\left(2 c_{3}-3 \ell_{12}\right) \alpha-c_{3}^{2}+\sqrt{\left(2 \alpha^{2}+\left(2 c_{3}-3 \ell_{12}\right) \alpha-c_{3}^{2}\right)^{2}+4\left(c_{3}-\alpha\right)^{2}\left(3 \ell_{12} c_{3}-\left(c_{3}^{2}+c_{3} \alpha+\alpha^{2}\right)\right)}}{2\left(c_{3}-\alpha\right)^{2}}$

The function $g_{\mathcal{V}}$ is well defined, positive and decreasing on $\left[0, f_{\mathcal{V}}^{12}\left(c_{3}\right)\right]$. It verifies the identities $g_{\mathcal{V}}(0)=f_{\mathcal{V}}^{23}\left(c_{1}\right)$ and $g_{\mathcal{V}}\left(f_{\mathcal{V}}^{12}\left(c_{3}\right)\right)=0$. We also introduce $h_{\mathcal{V}}^{13}$ as:

$$
h_{\mathcal{V}}^{13}(\alpha)=c_{1} \frac{2 \alpha^{3}+3\left(c_{3}-\alpha\right)\left(c_{3}\left(c_{3}-\ell_{12}\right)+\ell_{12} \alpha\right)-\sqrt{3\left(c_{3}-\alpha\right)^{2}\left(3\left(c_{3}\left(c_{3}-\ell_{12}\right)+\ell_{12} \alpha\right)^{2}+4\left(c_{3}-\ell_{12}\right) \alpha^{3}\right)}}{2 \alpha\left(\alpha^{2}+3 c_{3}\left(c_{3}-\alpha\right)\right)}
$$

The function $h_{\mathcal{V}}^{13}$ is well defined, positive and monotonically increasing on $\left[0, c_{3}\right]$. It verifies the identities $h_{\mathcal{V}}^{13}(0)=0$ and $h_{\mathcal{V}}^{13}\left(c_{3}\right)=c_{1}$. The angles of the local chart of figure 5 are given by:

$$
\begin{aligned}
\theta_{1}=\arctan \left(\frac{\ell_{23}}{c_{2}}\right) \quad \theta_{2}^{\mathrm{t}} & =\arctan \left(\frac{3 \ell_{23}}{c_{2}}\right) \quad \theta_{3}=\arctan \left(\frac{c_{1}}{c_{2}}\right) \quad \theta_{4}^{\mathrm{t}}=\arctan \left(\frac{c_{1}}{3 \ell_{13}}\right) \quad \theta_{5}=\arctan \left(\frac{c_{1}}{\ell_{13}}\right) \\
\theta_{2}^{\mathrm{h}} & =\arctan \left(\frac{c_{1}-f_{\mathcal{V}}^{23}\left(c_{1}\right)}{c_{2}}\right) \quad \theta_{4}^{\mathrm{h}}=\arctan \left(\frac{c_{1}}{c_{2}-f_{\mathcal{V}}^{13}\left(c_{2}\right)}\right)
\end{aligned}
$$

Consider the third-degree polynomial $x^{3}+p x+q=0$. When the discriminant $\Delta=4 p^{3}+27 q^{2} \leq 0$, the polynomial has three real roots given by $(k \in\{0,1,2\})$ :

$$
x_{k}=2 \sqrt{\frac{-p}{3}} \cos \left(\frac{1}{3} \arccos \left(\frac{-q}{2 \sqrt{\left(-\frac{p}{3}\right)^{3}}}\right)+\frac{2 k \pi}{3}\right)
$$


Appendix C.2. Parametrization of the Triangle configuration

Appendix C.2.1. Parametrization in $(\alpha, \gamma)$
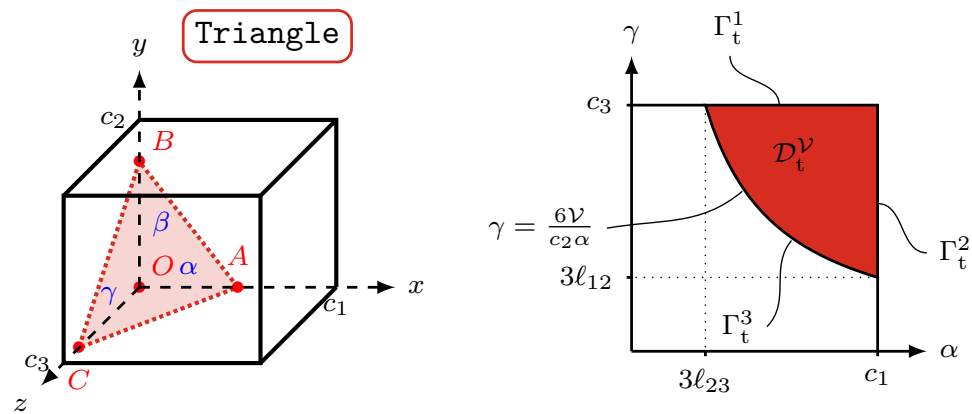

Figure C.12: Triangle configuration (left) and definition domain $\mathcal{D}_{\mathrm{t}}^{\mathcal{V}}$ with $\chi<\frac{1}{6}$ (right).

In the Triangle configuration, the plane - the surface $\mathfrak{P}$ defined in (C.2) — intersects the cell as presented on the left of figure C.12. The coordinates of the points are given by $A=(\alpha, 0,0), B=(0, \beta, 0)$, and $C=(0,0, \gamma)$. The coefficients of the plane (C.2) are given by:

$$
a_{1}=\frac{1}{\alpha} \quad a_{2}=\frac{1}{\beta} \quad a_{3}=\frac{1}{\gamma}
$$

The volume of the tetrahedron $O A B C$ is given by $\mathcal{V}_{\mathrm{t}}(\alpha, \beta, \gamma)=\frac{\alpha \beta \gamma}{6}$. Its centroid is given by $\mathcal{C}_{\mathrm{t}}(\alpha, \beta, \gamma)=$ $\frac{1}{4}[\alpha, \beta, \gamma]$. By imposing the fixed reference volume $\mathcal{V}$, we obtain:

$$
\beta_{\mathrm{t}}(\alpha, \gamma, \mathcal{V})=\frac{6 \mathcal{V}}{\alpha \gamma} \quad \mathcal{C}_{\mathrm{t}}^{\mathcal{V}}(\alpha, \gamma)=\frac{1}{4}\left[\alpha, \frac{6 \mathcal{V}}{\alpha \gamma}, \gamma\right]
$$

In closed form, the centroid verifies the equation $Y=\frac{3 \mathcal{V}}{32 X Z}$ which is a portion of a hyperboloid. When the parameters $\alpha \in\left[0, c_{1}\right]$ and $\gamma \in\left[0, c_{3}\right]$ are fixed, we have $\beta_{\mathrm{t}} \in\left[0, c_{2}\right]$, so, $0 \leq \frac{6 \mathcal{V}}{\alpha \gamma} \leq c_{2}$. The domain $\mathcal{D}_{\mathrm{t}}^{\mathcal{V}}$, represented on figure C.12, is given by:

$$
\mathcal{D}_{\mathrm{t}}^{\mathcal{V}}=\left\{(\alpha, \gamma) \in\left[0, c_{1}\right] \times\left[0, c_{3}\right] \mid \gamma \geq \frac{6 \mathcal{V}}{c_{2} \alpha}\right\}
$$

Its three limit curves are given by $\Gamma_{\mathrm{t}}^{3}: \alpha \mapsto\left(\alpha, \frac{6 \mathcal{V}}{c_{2} \alpha}\right)$ with $\alpha \in\left[3 \ell_{23}, c_{1}\right], \Gamma_{\mathrm{t}}^{2}: \gamma \mapsto\left(c_{1}, \gamma\right)$ with $\gamma \in\left[3 \ell_{12}, c_{3}\right]$ and $\Gamma_{\mathrm{t}}^{1}: \alpha \mapsto\left(\alpha, c_{3}\right)$ with $\alpha \in\left[3 \ell_{23}, c_{1}\right]$.

Appendix C.2.2. Parametrization of the centroid and the limit curves in $(\theta, \phi)$

From equations (C.3), (C.10) and relation (C.11), we get $\tan (\theta)=\frac{\alpha}{\beta}=\frac{\alpha^{2} \gamma}{6 \mathcal{V}}$ and $\cot (\phi)=\frac{\alpha}{\gamma} \cos (\theta)$. We solve these equations for $\alpha$ and $\gamma$ to obtain:

$$
\gamma_{\mathrm{t}}(\theta, \phi, \mathcal{V})=\left(\frac{6 \mathcal{V} \tan (\theta)}{(\cot (\phi) \sec (\theta))^{2}}\right)^{\frac{1}{3}} \quad \alpha_{\mathrm{t}}(\theta, \phi, \mathcal{V})=\gamma_{\mathrm{t}}(\theta, \phi, \mathcal{V}) \cot (\phi) \sec (\theta)
$$

We obtain the final parametrization (17) by replacing $\alpha$ and $\gamma$ in (C.11) by their expression given in (C.12).

The limit curves in $(\theta, \phi)$ are obtained from the limit curves in $(\alpha, \gamma)$. For the curve $\Gamma_{\mathrm{t}}^{3}$, we have $\gamma=\frac{6 \mathcal{V}}{c_{2} \alpha}$. Hence, $\tan (\theta)=\frac{\alpha}{c_{2}}$ and $\cot (\phi)=\frac{c_{2} \alpha^{2}}{6 \mathcal{V}} \cos (\theta)$. When solved for $(\theta, \phi)$, we obtain $\phi_{\mathrm{t} 3}^{\lim }(\theta)(12)$ defined on $\left[\theta_{2}^{\mathrm{t}}, \theta_{3}\right]$. For the curve $\Gamma_{\mathrm{t}}^{2}$, we have $\alpha=c_{1}$. Hence, $\tan (\theta)=\frac{c_{1}^{2} \gamma}{6 \mathcal{V}}$ and $\cot (\phi)=\frac{c_{1}}{\gamma} \cos (\theta)$. We obtain $\phi_{\mathrm{t} 2}^{\lim }(\theta)$ (12) defined on $\left[\theta_{3}, \theta_{4}^{\mathrm{t}}\right]$. For the curve $\Gamma_{\mathrm{t}}^{1}$, we have $\gamma=c_{3}$. Hence, $\tan (\theta)=\frac{c_{3} \alpha^{2}}{6 \mathcal{V}}$ and $\cot (\phi)=\frac{\alpha}{c_{3}} \cos (\theta)$. We obtain $\phi_{\mathrm{t} 1}^{\lim }(\theta)(12)$ defined on $\left[\theta_{2}^{\mathrm{t}}, \theta_{4}^{\mathrm{t}}\right]$. 
Appendix C.3. Parametrization of the QuadFaceL configuration

Appendix C.3.1. Parametrization in $(\alpha, \gamma)$
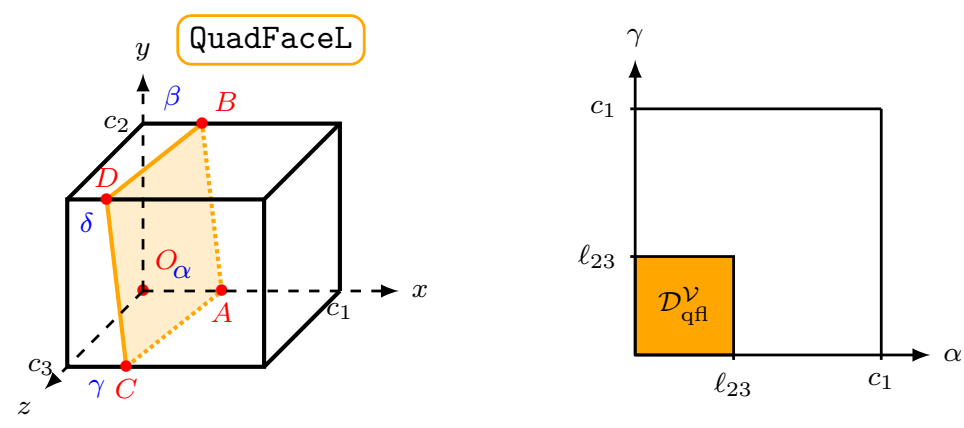

Figure C.13: QuadFaceL configuration (left) and definition domain $\mathcal{D}_{\mathrm{qf}}^{\mathcal{V}}$ (right).

In the QuadFaceL configuration, the plane intersects the cell as presented on the left of figure C.13. The coordinates of the points are given by $A=(\alpha, 0,0), B=\left(\beta, c_{2}, 0\right), C=\left(\gamma, 0, c_{3}\right)$, and $D=\left(\delta, c_{2}, c_{3}\right)$. The coefficients of the plane (C.2) are given by:

$$
a_{1}=\frac{1}{\alpha} \quad a_{2}=\frac{\alpha-\beta}{c_{2} \alpha} \quad a_{3}=\frac{\alpha-\gamma}{c_{3} \alpha}
$$

Since the point $D$ belongs to the plane, we have $\delta=\beta+\gamma-\alpha$. The plane can be parametrized by $x=\zeta_{\mathrm{qfl}}(y, z)=\alpha+\frac{\beta-\alpha}{c_{2}} y+\frac{\gamma-\alpha}{c_{3}} z$. The volume of the approximation is given by:

$$
\mathcal{V}_{\mathrm{qfl}}(\alpha, \beta, \gamma)=\int_{0}^{c_{3}} \int_{0}^{c_{2}} \int_{0}^{\zeta_{\mathrm{qfl}}(y, z)} 1 \mathrm{~d} x \mathrm{~d} y \mathrm{~d} z=\frac{c_{2} c_{3}(\beta+\gamma)}{2}
$$

The centroid of the approximation is given by:

$$
\mathcal{C}_{\mathrm{qfl}}(\alpha, \beta, \gamma)=\frac{1}{\mathcal{V}_{\mathrm{qf}}(\alpha, \beta, \gamma)} \int_{0}^{c_{3}} \int_{0}^{c_{2}} \int_{0}^{\zeta_{\mathrm{qfl}}(y, z)}\left[\begin{array}{l}
x \\
y \\
z
\end{array}\right] \mathrm{d} x \mathrm{~d} y \mathrm{~d} z=\frac{1}{6(\beta+\gamma)}\left[\begin{array}{c}
2 \gamma^{2}+3 \beta \gamma+2 \beta^{2}-\alpha(\beta+\gamma)+\alpha^{2} \\
c_{2}(3 \gamma+4 \beta-\alpha) \\
c_{3}(4 \gamma+3 \beta-\alpha)
\end{array}\right]
$$

By imposing the fixed reference volume $\mathcal{V}$ in (C.14), we obtain:

$$
\beta_{\mathrm{qfl}}(\alpha, \gamma, \mathcal{V})=\ell_{23}-\gamma \quad \mathcal{C}_{\mathrm{qf}}^{\mathcal{V}}(\alpha, \gamma)=\frac{1}{6 \ell_{23}}\left[\begin{array}{c}
2\left(\ell_{23}\right)^{2}-\ell_{23}(\alpha+\gamma)+\alpha^{2}+\gamma^{2} \\
c_{2}\left(4 \ell_{23}-(\alpha+\gamma)\right) \\
c_{3}\left(3 \ell_{23}-(\alpha-\gamma)\right)
\end{array}\right]
$$

In closed form, the centroid verifies the equation $X=\frac{\mathcal{V}}{2 c_{2}^{3} c_{3}^{3}}\left(12 c_{3}^{2}\left(Y-\frac{c_{2}}{2}\right)^{2}+12 c_{2}^{2}\left(Z-\frac{c_{3}}{2}\right)^{2}+c_{2}^{2} c_{3}^{2}\right)$ which is a portion of a paraboloid. When the parameters $\alpha, \gamma \in\left[0, c_{1}\right]$ are fixed, we have $\beta_{\mathrm{qff}}, \delta_{\mathrm{qff}} \in\left[0, c_{1}\right]$, so $0 \leq \ell_{23}-\gamma \leq c_{1}$ and $0 \leq \ell_{23}-\alpha \leq c_{1}$. Hence, using the relation $\ell_{23} \leq c_{1}$ (as $\left.\mathcal{V} \leq c_{1} c_{2} c_{3} / 2\right)$, we get $(\alpha, \gamma) \in\left[0, \ell_{23}\right]^{2}$. The definition domain $\mathcal{D}_{\mathrm{qfl}}^{\mathcal{V}}$, represented on figure C.13, is then given by:

$$
\mathcal{D}_{\mathrm{qf}}^{\mathcal{V}}=\left\{(\alpha, \gamma) \in\left[0, c_{1}\right]^{2} \mid \gamma \leq \ell_{23} \text { and } \alpha \leq \ell_{23}\right\}
$$

Appendix C.3.2. Parametrization of the centroid in $(\theta, \phi)$

From (C.3), (C.13) and relation (C.15), we obtain $\tan (\theta)=\frac{\alpha-\beta}{c_{2}}=\frac{\alpha+\gamma-\ell_{23}}{c_{2}}$ and $\cot (\phi)=\frac{\alpha-\gamma}{c_{3}} \cos (\theta)$. We solve these equations for $\alpha$ and $\gamma$ to obtain the following relations:

$$
\gamma_{\mathrm{qf}}(\theta, \phi, \mathcal{V})=\frac{1}{2}\left(c_{2} \tan (\theta)+\ell_{23}-c_{3} \cot (\phi) \sec (\theta)\right) \quad \alpha_{\mathrm{qfl}}(\theta, \phi, \mathcal{V})=\gamma_{\mathrm{qf}}(\theta, \phi, \mathcal{V})+c_{3} \cot (\phi) \sec (\theta)
$$

After simplifications, we obtain the final parametrization (18a) by replacing $\alpha$ and $\gamma$ in (C.15) with their expression given in (C.16). For the limit curves, we will use those given by the Penta configuration. 
Appendix C.4. Parametrization of the QuadEdgeL configuration

Appendix C.4.1. Parametrization in $(\alpha, \gamma)$

In the QuadEdgeL configuration, the plane intersects the cell as presented on figure C.14. The coordinates of the points are given by $A=(\alpha, 0,0), B=(0, \beta, 0), C=\left(\gamma, 0, c_{3}\right)$, and $D=\left(0, \delta, c_{3}\right)$. The coefficients of the plane (C.2) are given by:

$$
a_{1}=\frac{1}{\alpha} \quad a_{2}=\frac{1}{\beta} \quad a_{3}=\frac{\alpha-\gamma}{c_{3} \alpha}
$$

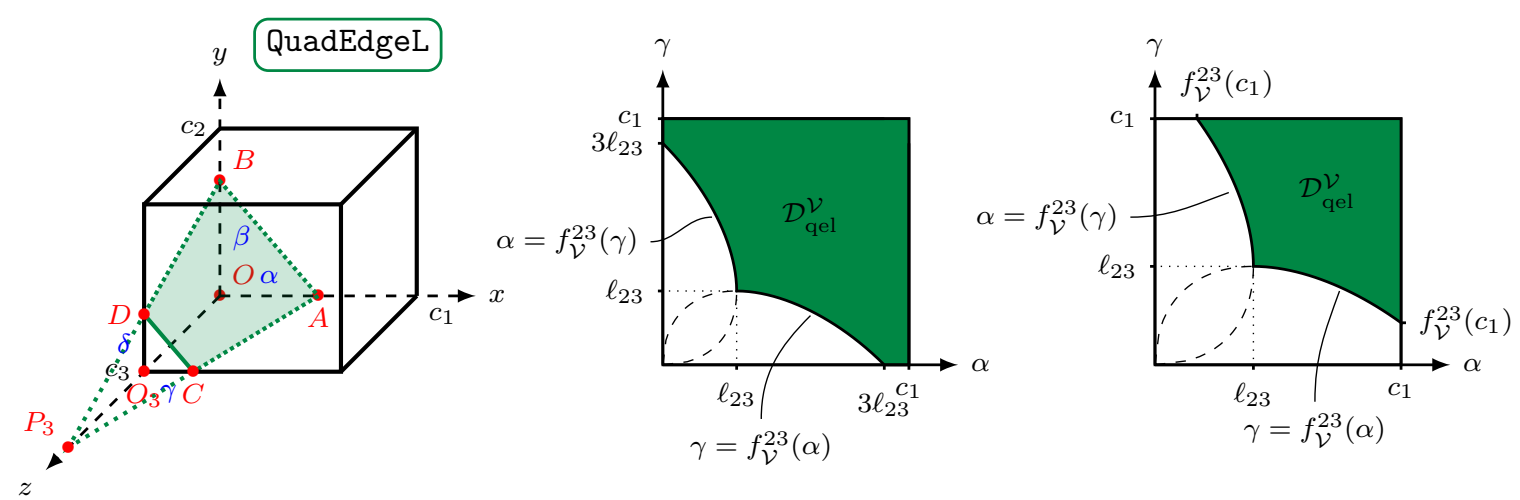

Figure C.14: QuadEdgeL configuration (left), definition domain $\mathcal{D}_{\text {qel }}^{\mathcal{V}}$ for $\chi<\frac{1}{6}$ (middle) and for $\chi>\frac{1}{6}$ (right).

Since point $D$ belongs to plane $\mathfrak{P}$, we have $\delta=\frac{\beta \gamma}{\alpha}$. We introduce the points $O_{3}=\left(0,0, c_{3}\right)$ and $P_{3}=\left(0,0, h_{3}\right)$ where $h_{3}=\frac{c_{3} \alpha}{\alpha-\gamma}$. The last point corresponds to the intersection of the planes $\{x=0\},\{y=0\}$, and the interface. The volume and the centroid of the domain $O A B O_{3} C D$ are computed using the additivity of the volume and the first momentum of the tetrahedrons $O A B P_{3}$ and $O_{3} C D P_{3}$, which gives:

$$
\mathcal{V}_{\mathrm{qel}}(\alpha, \beta, \gamma)=\frac{c_{3} \beta\left(\alpha^{2}+\alpha \gamma+\gamma^{2}\right)}{6 \alpha} \quad \mathcal{C}_{\mathrm{qel}}(\alpha, \beta, \gamma)=\left[\begin{array}{c}
\frac{\alpha^{3}+\alpha^{2} \gamma+\alpha \gamma^{2}+\gamma^{3}}{4\left(\alpha^{2}+\alpha \gamma+\gamma^{2}\right)} \\
\frac{\beta\left(\alpha^{3}+\alpha^{2} \gamma+\alpha \gamma^{2}+\gamma^{3}\right)}{4 \alpha\left(\alpha^{2}+\alpha \gamma+\gamma^{2}\right)} \\
\frac{c_{3}\left(3 \gamma^{2}+2 \alpha \gamma+\alpha^{2}\right)}{4\left(\alpha^{2}+\alpha \gamma+\gamma^{2}\right)}
\end{array}\right]
$$

Note that the volume and the centroid are continuous between the QuadEdgeL and the Triangle configurations since $\mathcal{V}_{\text {qel }}(\alpha, \beta, 0)=\mathcal{V}_{\mathrm{t}}\left(\alpha, \beta, c_{3}\right)$ and $\mathcal{C}_{\mathrm{qel}}(\alpha, \beta, 0)=\mathcal{C}_{\mathrm{t}}\left(\alpha, \beta, c_{3}\right)$. By imposing a fixed reference volume $\mathcal{V}$ in (C.18), we get:

$$
\beta_{\mathrm{qel}}(\alpha, \gamma, \mathcal{V})=\frac{6 \mathcal{V} \alpha}{c_{3}\left(\alpha^{2}+\alpha \gamma+\gamma^{2}\right)}
$$

When the parameters $\alpha, \gamma \in\left[0, c_{1}\right]$ are fixed, we have $\beta_{\mathrm{qel}} \in\left[0, c_{2}\right]$ so $0 \leq \frac{6 \mathcal{V} \alpha}{c_{3}\left(\alpha^{2}+\alpha \gamma+\gamma^{2}\right)} \leq c_{2}$. The right inequality writes $\gamma^{2}+\alpha \gamma+\alpha^{2}-3 \ell_{23} \alpha \geq 0$ or equivalently $\gamma \geq f_{\mathcal{V}^{2}}^{23}(\alpha)$ using (C.6) where we keep the positive solution because $\gamma \geq 0$. We have also $\delta_{\text {qel }} \in\left[0, c_{2}\right]$ so $0 \leq \frac{6 \mathcal{V} \gamma}{c_{3}\left(\alpha^{2}+\alpha \gamma+\gamma^{2}\right)} \leq c_{2}$ and hence we obtained similarly that $\alpha \geq f_{\mathcal{V}}^{23}(\gamma)$. The definition domain $\mathcal{D}_{\text {qel }}^{\mathcal{V}}$ represented in figure C.14 is then given by:

$$
\mathcal{D}_{\mathrm{qel}}^{\mathcal{V}}=\left\{(\alpha, \gamma) \in\left[0, c_{1}\right]^{2} \mid \gamma \geq f_{\mathcal{V}}^{23}(\alpha) \text { and } \alpha \geq f_{\mathcal{V}}^{23}(\gamma)\right\}
$$

Appendix C.4.2. Parametrization of the centroid in $(\theta, \phi)$

From equations (C.3), (C.17) and relation (C.19), we get $\tan (\theta)=\frac{\alpha}{\beta}=\frac{c_{3}\left(\alpha^{2}+\alpha \gamma+\gamma^{2}\right)}{6 \mathcal{V}} \operatorname{and} \cot (\phi)=$ $\frac{\alpha-\gamma}{c_{3}} \cos (\theta)$. We obtain a second degree polynomial equation in $\gamma$ and we get the following relations - keeping the positive root - with $T_{2}^{l}=c_{2} \tan (\theta)$ and $T_{3}^{l}=c_{3} \cot (\phi) \sec (\theta)$ :

$$
\gamma_{\mathrm{qel}}(\theta, \phi, \mathcal{V})=\frac{1}{6}\left(-3 T_{3}^{l}+\sqrt{36 \ell_{23} T_{2}^{l}-3\left(T_{3}^{l}\right)^{2}}\right) \quad \alpha_{\mathrm{qel}}(\theta, \phi, \mathcal{V})=\gamma_{\mathrm{qel}}(\theta, \phi, \mathcal{V})+T_{3}^{l}
$$


After simplifications, we obtain the final parametrization (19) by replacing $\alpha, \beta$, and $\gamma$ in (C.18) by their expressions given in (C.19) and (C.20).

Appendix C.5. Parametrization of the PentaL configuration

Appendix C.5.1. Parametrization in $(\alpha, \gamma)$
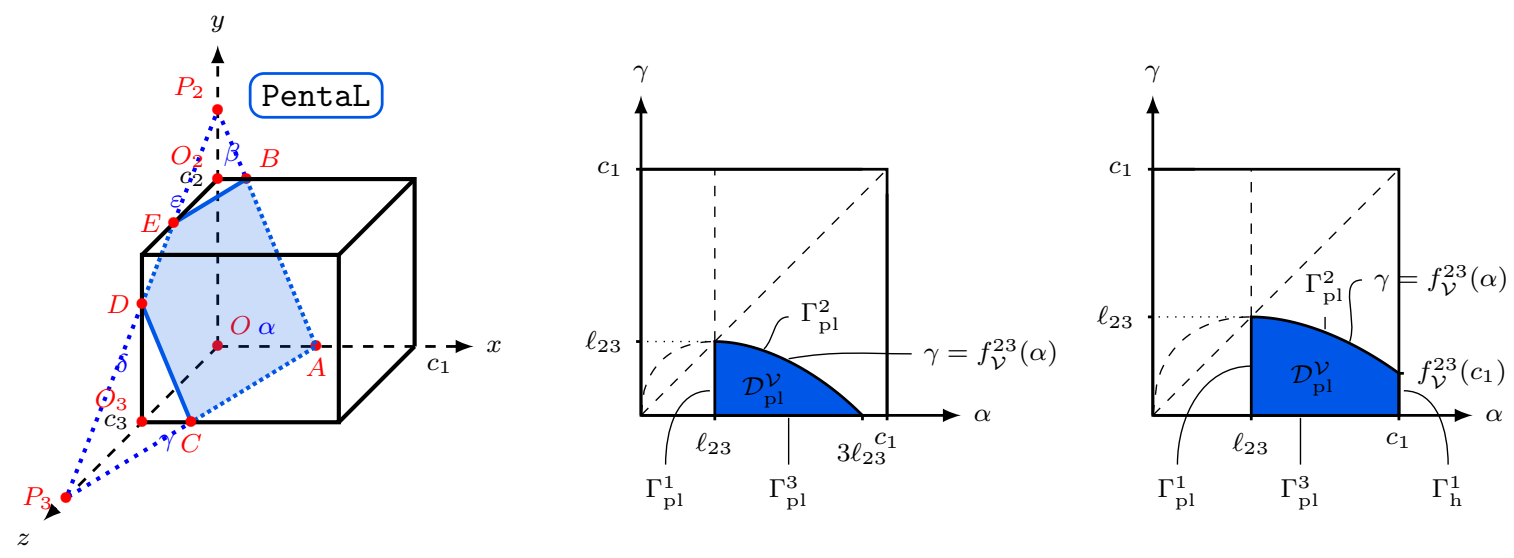

Figure C.15: PentaL configuration (left), definition domain $\mathcal{D}_{\mathrm{pl}}^{\mathcal{V}}$ with $\chi<\frac{1}{6}$ (middle) and $\chi>\frac{1}{6}$ (right).

In the PentaL configuration, the plane intersects the cell as presented on the left of figure C.15. The coordinates of the points are given by $A=(\alpha, 0,0), B=\left(\beta, c_{2}, 0\right), C=\left(\gamma, 0, c_{3}\right), D=\left(0, \delta, c_{3}\right)$, and $E=\left(0, c_{2}, \varepsilon\right)$. The coefficients of the plane (C.2) are given by:

$$
a_{1}=\frac{1}{\alpha} \quad a_{2}=\frac{\alpha-\beta}{c_{2} \alpha} \quad a_{3}=\frac{\alpha-\gamma}{c_{3} \alpha}
$$

Since the points $D$ and $E$ belong to the plane, we have $\delta=\frac{c_{2} \gamma}{\alpha-\beta}$ and $\varepsilon=\frac{c_{3} \beta}{\alpha-\gamma}$. Furthermore, we have $\alpha, \beta, \gamma \in\left[0, c_{1}\right] . \delta \in\left[0, c_{2}\right], \varepsilon \in\left[0, c_{3}\right]$, thus $0 \leq \frac{c_{2} \gamma}{\alpha-\beta} \leq c_{2}$. The left inequality is satisfied if $\alpha \geq \beta$ and the right one is satisfied if $\alpha \geq \beta+\gamma$. We also have $0 \leq \frac{c_{3} \beta}{\alpha-\gamma} \leq c_{3}$. The left inequality is satisfied if $\alpha \geq \gamma$ and the right one is satisfied if $\alpha \geq \beta+\gamma$. Therefore, only one inequality stands:

$$
\beta \leq \alpha-\gamma
$$

We introduce the points $O_{3}=\left(0,0, c_{3}\right), O_{2}=\left(0, c_{2}, 0\right)$, and $P_{2}=\left(0, h_{2}, 0\right)$ where $h_{2}=\frac{c_{2} \alpha}{\alpha-\beta}$. The point $P_{2}$ is the intersection of the planes $\{x=0\},\{z=0\}$ and the interface $\mathfrak{P}$. We also introduce the point $P_{3}=\left(0,0, h_{3}\right)$ where $h_{3}=\frac{c_{3} \alpha}{\alpha-\gamma}$ as the intersection of the planes $\{x=0\},\{y=0\}$ and the interface $\mathfrak{P}$. The volume and the centroid of the domain $\mathrm{OABO}_{2} \mathrm{EDCO}_{3}$ are computed using the additivity of the volume and the first momentum of the tetrahedrons $O A P_{2} P_{3}, O_{2} B E P_{2}$ and $O_{3} C D P_{3}$, so:

$$
\mathcal{V}_{\mathrm{pl}}(\alpha, \beta, \gamma)=\frac{c_{2} c_{3}\left(\alpha^{3}-\left(\beta^{3}+\gamma^{3}\right)\right)}{6(\alpha-\beta)(\alpha-\gamma)} \quad \mathcal{C}_{\mathrm{pl}}(\alpha, \beta, \gamma)=\left[\begin{array}{c}
\frac{\alpha^{4}-\left(\beta^{4}+\gamma^{4}\right)}{4\left(\alpha^{3}-\left(\beta^{3}+\gamma^{3}\right)\right.} \\
\frac{c_{2}\left(\alpha^{4}-\left(\beta^{4}+\gamma^{4}\right)-4 \beta^{3}(\alpha-\beta)\right)}{4(\alpha-\beta)\left(\alpha^{3}-\left(\beta^{3}+\gamma^{3}\right)\right)} \\
\frac{c_{3}\left(\alpha^{4}-\left(\beta^{4}+\gamma^{4}\right)-4 \gamma^{3}(\alpha-\gamma)\right)}{4(\alpha-\gamma)\left(\alpha^{3}-\left(\beta^{3}+\gamma^{3}\right)\right)}
\end{array}\right]
$$

Note that the volume and the centroid are continuous between the QuadEdgeL and the PentaL configurations since $\beta_{\mathrm{pl}}=0$ is equivalent to $\beta_{\mathrm{qel}}=c_{2}$ and we verify that $\mathcal{V}_{\mathrm{pl}}(\alpha, 0, \gamma)=\mathcal{V}_{\mathrm{qel}}\left(\alpha, c_{2}, \gamma\right)$ and $\mathcal{C}_{\mathrm{pl}}(\alpha, 0, \gamma)=$ $\mathcal{C}_{\mathrm{qel}}\left(\alpha, c_{2}, \gamma\right)$. Similarly, the volume and the centroid are continuous between the PentaL and the QuadFaceL configurations since $\delta_{\mathrm{qf}}=\beta+\gamma-\alpha=0$ is equivalent to $\delta_{\mathrm{pl}}=\frac{c_{2} \gamma}{\alpha-\beta}=c_{2}$, that is $\beta_{\mathrm{pl}}=\beta_{\mathrm{qfl}}=\alpha-\gamma$, and by verifying that $\mathcal{V}_{\mathrm{pl}}(\alpha, \alpha-\gamma, \gamma)=\mathcal{V}_{\mathrm{qf}}(\alpha, \alpha-\gamma, \gamma)$ and $\mathcal{C}_{\mathrm{pl}}(\alpha, \alpha-\gamma, \gamma)=\mathcal{C}_{\mathrm{qfl}}(\alpha, \alpha-\gamma, \gamma)$. 
For a given reference fixed volume we get with (C.23) that $\beta$ is the root of the following third degree polynomial:

$$
\Psi_{\mathrm{pl}}(\alpha, \beta, \gamma)=\beta^{3}-3 \ell_{23}(\alpha-\gamma) \beta-(\alpha-\gamma)\left(\alpha^{2}+\alpha \gamma+\gamma^{2}-3 \ell_{23} \alpha\right)=0
$$

Let us find the definition domain of the Penta configuration:

$$
\mathcal{D}_{\mathrm{pl}}^{\mathcal{V}}=\left\{(\alpha, \gamma) \in\left[0, c_{1}\right]^{2} \mid 0 \leq \beta_{\mathrm{pl}}(\alpha, \gamma, \mathcal{V}) \leq c_{1} \text { and } \beta_{\mathrm{pl}}(\alpha, \gamma, \mathcal{V}) \leq \alpha-\gamma\right\}
$$

where $\beta_{\mathrm{pl}}(\alpha, \gamma, \mathcal{V})$ is solution of (C.24). To find this domain, let us first find its boundaries. If $\beta=c_{1}$, we have $c_{1} \geq \alpha \geq c_{1}+\gamma$ and therefore $(\alpha, \gamma)=\left(c_{1}, 0\right)$. With (C.24) and using (C.6) we have:

$$
\begin{array}{ll}
\text { For } \beta=0 & \Psi_{\mathrm{pl}}(\alpha, 0, \gamma)=(\gamma-\alpha)\left(\gamma-f_{\mathcal{V}}^{23}(\alpha)\right)\left(\gamma-\tilde{f}_{\mathcal{V}}^{23}(\alpha)\right)=0 \\
\text { For } \beta=\alpha-\gamma & \Psi_{\mathrm{pl}}(\alpha, \alpha-\gamma, \gamma)=3 \gamma(\alpha-\gamma)\left(\ell_{23}-\alpha\right)=0
\end{array}
$$

Therefore, the possible boundaries of the domain $\mathcal{D}_{\mathrm{pl}}^{\mathcal{V}}$ are necessary in the following list: $\alpha=0, \alpha=c_{1}, \gamma=0$, $\gamma=c_{1}, \gamma=\alpha, \gamma=f_{\mathcal{V}}^{23}(\alpha)$, or $\alpha=\ell_{23}$. The root $\gamma=\tilde{f}_{\mathcal{V}}^{23}(\alpha)$ is excluded since it is negative, so outside of $\left[0, c_{1}\right]^{2}$. These curves partition $\left[0, c_{1}\right]^{2}$ in six regions as represented in figure C.15. Thus $\mathcal{D}_{\mathrm{pl}}^{\mathcal{V}}$ is the reunion of some - possibly only one - of these regions. It is easy to check numerically by picking one value in these regions that five of them do not verify all the constraints. In each of these regions, the three roots of (C.24) verifies either $\beta \in \mathbb{C}$ or $\beta<0$ or $\beta>\alpha-\gamma$, which violates the conditions. Therefore the definition domain of the PentaL is necessarily given by the remaining region:

$$
\mathcal{D}_{\mathrm{pl}}^{\mathcal{V}}=\left\{(\alpha, \gamma) \in\left[0, c_{1}\right]^{2} \mid \alpha \geq \ell_{23} \text { and } \gamma \leq f_{\mathcal{V}}^{23}(\alpha)\right\}
$$

The limit curves are given by $\Gamma_{\mathrm{pl}}^{1}: \alpha \mapsto\left(\ell_{23}, \gamma\right)$ where $\gamma \in\left[0, \ell_{23}\right], \Gamma_{\mathrm{pl}}^{2}: \alpha \mapsto\left(\alpha, f_{\mathcal{V}}^{23}(\alpha)\right)$ where $\alpha \in$ $\left[\ell_{23}, \min \left(3 \ell_{23}, c_{1}\right)\right]$ and $\Gamma_{\mathrm{pl}}^{3}: \alpha \mapsto(\alpha, 0)$ where $\alpha \in\left[\ell_{23}, \min \left(3 \ell_{23}, c_{1}\right)\right]$. When $\chi>\frac{1}{6}$, there is another curve $\Gamma_{\mathrm{h}}^{1}: \gamma \mapsto\left(c_{1}, \gamma\right)$ common to the Hexa configuration where $\gamma \in\left[0, f_{\mathcal{V}}^{23}\left(c_{1}\right)\right]$.

In Appendix C.7.2, we have proved that the discriminant of the third-degree equation (C.24) is negative on $\mathcal{D}_{\mathrm{pl}}^{\mathcal{V}}$, so there are three real roots $\beta_{k}$ for $k \in \llbracket 0,2 \rrbracket$ that can be expressed by formula (C.9). In Appendix C.7.2, we have also shown that the only solution that verifies $0 \leq \beta \leq \alpha-\gamma$ on $\mathcal{D}_{\mathrm{pl}}^{\mathcal{V}}$ is the root for $k=2$ given by:

$$
\beta_{\mathrm{pl}}(\alpha, \gamma, \mathcal{V})=2 \sqrt{\ell_{23}(\alpha-\gamma)} \cos \left(\frac{1}{3} \arccos \left(\frac{\alpha^{2}+\alpha \gamma+\gamma^{2}-3 \ell_{23} \alpha}{2 \ell_{23} \sqrt{\ell_{23}(\alpha-\gamma)}}\right)+\frac{4 \pi}{3}\right)
$$

In the Penta configuration, we can find the following simpler formulas for the limits $\Gamma_{\mathrm{pl}}^{1}, \Gamma_{\mathrm{pl}}^{2}$ and $\Gamma_{\mathrm{pl}}^{3}$ :

$$
\begin{array}{lrl}
\text { For } \Gamma_{\mathrm{pl}}^{1} & \forall \gamma \in\left[0, \ell_{23}\right], & \beta_{\mathrm{pl}}\left(\ell_{23}, \gamma, \mathcal{V}\right)=\ell_{23}-\gamma \\
\text { For } \Gamma_{\mathrm{pl}}^{2} & \forall \alpha \in\left[\ell_{23}, \min \left(3 \ell_{23}, c_{1}\right)\right], & \beta_{\mathrm{pl}}\left(\alpha, f_{\mathcal{V}}^{23}(\alpha), \mathcal{V}\right)=0 \\
\text { For } \Gamma_{\mathrm{pl}}^{3} & \forall \alpha \in\left[\ell_{23}, \min \left(3 \ell_{23}, c_{1}\right)\right], & \beta_{\mathrm{pl}}(\alpha, 0, \mathcal{V})=f_{\mathcal{V}}^{23}(\alpha)
\end{array}
$$

When $\chi>\frac{1}{6}$, we did not find a simpler expression of the fourth limit $\Gamma_{\mathrm{h}}^{1}$.

To obtain these formulas, we insert the equations of the limits into equation (C.24):

$$
\begin{array}{ll}
\text { On } \Gamma_{\mathrm{pl}}^{1} & \Psi_{\mathrm{pl}}\left(\ell_{23}, \beta, \gamma\right)=\left(\beta-f_{\mathcal{V}}^{23}\left(\ell_{23}-\gamma\right)\right)\left(\beta-\tilde{f}_{\mathcal{V}}^{23}\left(\ell_{23}-\gamma\right)\right)\left(\beta-\left(\ell_{23}-\gamma\right)\right) \\
\text { On } \Gamma_{\mathrm{pl}}^{2} & \Psi_{\mathrm{pl}}\left(\alpha, \beta, f_{\mathcal{V}}^{23}(\alpha)\right)=\left(\beta-\sqrt{3 \ell_{23}\left(\alpha-f_{\mathcal{V}}^{23}(\alpha)\right)}\right)\left(\beta+\sqrt{3 \ell_{23}\left(\alpha-f_{\mathcal{V}}^{23}(\alpha)\right)}\right)(\beta-0) \\
\text { On } \Gamma_{\mathrm{pl}}^{3} & \Psi_{\mathrm{pl}}(\alpha, \beta, 0)=(\beta-\alpha)\left(\beta-\tilde{f}_{\mathcal{V}}^{23}(\alpha)\right)\left(\beta-f_{\mathcal{V}}^{23}(\alpha)\right)
\end{array}
$$

On each boundaries, there are three roots for $\beta$. It can proved by a direct computation that the first root of each expression corresponds to the root $k=0$ of (C.24). The second term in each expression is negative since $\tilde{f}_{\mathcal{V}}^{23}<0$, so it corresponds to the root $k=1$ which is always negative as proved in proposition 1 . Therefore the third term in each expression is the root $k=2$ that we are looking for. 
Appendix C.5.2. Parametrization of the centroid and the limit curves in $(\theta, \phi)$

From equations (C.3) and (C.21), we get $\tan (\theta)=\frac{\alpha-\beta}{c_{2}}$ and $\cot (\phi)=\frac{\alpha-\gamma}{c_{3}} \cos (\theta)$. These non-linear equations are difficult to solve directly because of the expression of $\beta$ in (C.27). Instead, we express $\alpha$ and $\gamma$ as a function of $\beta, \theta$, and $\phi$ to obtain a third degree polynomial on $\beta$ using the expression of the volume (C.23). We introduce the following notations:

$$
\alpha=\beta+T_{2}^{l} \quad \gamma=\beta+T_{2}^{l}-T_{3}^{l} \quad \text { where } \quad T_{2}^{l}=c_{2} \tan (\theta) \quad T_{3}^{l}=c_{3} \cot (\phi) \sec (\theta)
$$

We replace these expressions in (C.23) to obtain the following third degree polynomial equation on $\beta$ :

$$
\left(\beta-T_{3}^{l}\right)^{3}-6 T_{2}^{l} T_{3}^{l}\left(\beta-T_{3}^{l}\right)-3 T_{2}^{l} T_{3}^{l}\left(T_{2}^{l}+T_{3}^{l}-\ell_{23}\right)=0
$$

We already know that the three roots are real. To find the suitable root, we can extend the proposition 1 to the case where $0 \leq X \leq 1$. In this case, $\beta_{0} \geq 0$ and $\beta_{1}, \beta_{2} \leq 0$. Note that we have to compute the lines $\left\{Y=-\frac{1}{2}\right\}$ and $\{\Psi=0\}$ which are crucial for the inequalities. Using this extended proposition with $\mathcal{K}=0$, $\mathcal{K}=c_{1}$ and $\mathcal{K}=\alpha-\gamma$, we can show that the only solution that verifies $\beta \leq \alpha-\gamma, 0 \leq \alpha \leq c_{1}, 0 \leq \beta \leq c_{1}$, and $0 \leq \gamma \leq c_{1}$ is given by the root $k=2$. The root can be expressed by:

$$
\beta_{\mathrm{pl}}(\theta, \phi, \mathcal{V})=T_{3}^{l}+2 \sqrt{2 T_{2}^{l} T_{3}^{l}} \mathcal{X}_{\mathrm{pl}} \quad \mathcal{X}_{\mathrm{pl}}=\cos \left(\frac{1}{3} \arccos \left(\frac{3\left(T_{2}^{l}+T_{3}^{l}-\ell_{23}\right)}{4 \sqrt{2 T_{2}^{l} T_{3}^{l}}}\right)+\frac{4 \pi}{3}\right)
$$

where $\mathcal{X}_{\mathrm{pl}}$ verifies the following equation:

$$
\left(\mathcal{X}_{\mathrm{pl}}\right)^{3}-\frac{3}{4} \mathcal{X}_{\mathrm{pl}}-\frac{3\left(T_{2}^{l}+T_{3}^{l}-\ell_{23}\right)}{16 \sqrt{2 T_{2}^{l} T_{3}^{l}}}=0
$$

To obtain the final parametrization (21), we replace $\alpha, \beta$ and $\gamma$ in (C.23) by their expressions given in (C.32) and (C.34). We obtain a fourth degree polynomial in $\mathcal{X}_{\mathrm{pl}}$ for each component. Then we use equation (C.35) to get a second degree polynomial in each component. Finally, after some simplifications, we obtain equation (21).

To compute the partial derivatives of the centroid using the formula of Chen \& Zhang [19], use the coordinates of the vertices of the interface given in order by $A=\left(\alpha_{\mathrm{pl}}, 0,0\right), B=\left(\beta_{\mathrm{pl}}, c_{2}, 0\right), E=\left(0, c_{2}, \varepsilon_{\mathrm{pl}}\right)$, $D=\left(0, \delta_{\mathrm{pl}}, c_{3}\right)$, and $C=\left(\gamma_{\mathrm{pl}}, 0, c_{3}\right)$ using $(\mathrm{C} .34)$ and the relations:

$$
\alpha_{\mathrm{pl}}=\beta_{\mathrm{pl}}+T_{2}^{l} \quad \gamma_{\mathrm{pl}}=\alpha_{\mathrm{pl}}-T_{3}^{l} \quad \delta_{\mathrm{pl}}=\frac{c_{2} \gamma_{\mathrm{pl}}}{T_{2}^{l}} \quad \varepsilon_{\mathrm{pl}}=\frac{c_{3} \beta_{\mathrm{pl}}}{T_{3}^{l}}
$$

For the PentaR (respectively PentaB) configuration, use a circular permutation of the coordinates and replace $T_{i}^{l}$ by $T_{i}^{r}$ (respectively $T_{i}^{b}$ ).

For the limit curve $\Gamma_{\mathrm{pl}}^{1}, \alpha=\ell_{23}$ hence, with formulas (C.28), we have $c_{2} \tan (\theta)=\ell_{23}-\beta_{\mathrm{pl}}\left(\ell_{23}, \gamma, \mathcal{V}\right)=\gamma$ and $c_{3} \cot (\phi)=\left(\ell_{23}-\gamma\right) \cos (\theta)$. We obtain $\phi_{\mathrm{pl} 1}^{\lim }(\theta)(13 \mathrm{a})$ defined on $\left[0, \theta_{1}\right]$. For the limit curve $\Gamma_{\mathrm{pl}}^{2}, \gamma=f_{\mathcal{V}}^{23}(\alpha)$ hence, with formulas (C.28), we have $c_{2} \tan (\theta)=\alpha-\beta_{\mathrm{pl}}\left(\alpha, f_{\mathcal{V}}^{23}(\alpha), \mathcal{V}\right)=\alpha$ and $c_{3} \cot (\phi)=\left(\alpha-f_{\mathcal{V}}^{23}(\alpha)\right) \cos (\theta)$. We obtain $\phi_{\mathrm{pl} 2}^{\lim }(\theta)(13 \mathrm{~b})$ defined on $\left[\theta_{1}, \theta_{2}^{\mathrm{t}}\right]$. If $\chi>\frac{1}{6}$, we have $\alpha \in\left[\ell_{23}, c_{1}\right]$. Hence $\phi_{\mathrm{pl} 2}^{\lim }(\theta)$ is defined on $\left[\theta_{1}, \theta_{3}\right]$. For the limit curve $\Gamma_{\mathrm{pl}}^{3}$, we have $\gamma=0$ hence, with equations (C.28), we have $c_{2} \tan (\theta)=\alpha-\beta_{\mathrm{pl}}(\alpha, 0, \mathcal{V})=$ $\alpha-f_{\mathcal{V}}^{23}(\alpha)$ and $c_{3} \cot (\phi)=\alpha \cos (\theta)$. The equation $\alpha-f_{\mathcal{V}}^{23}(\alpha)=T_{2}^{l}$ is a second degree polynomial in $\alpha$. We obtain the limit $\phi_{\mathrm{pl} 3}^{\lim }(\theta)(13 \mathrm{c})$ defined on $\left[0, \theta_{2}^{\mathrm{t}}\right]$. If $\chi>\frac{1}{6}$, we have $\alpha \in\left[\ell_{23}, c_{1}\right]$. Hence $\phi_{\mathrm{pl} 3}^{\lim }(\theta)$ is defined on $\left[0, \theta_{2}^{\mathrm{h}}\right]$.

For the limit curve $\Gamma_{\mathrm{h}}^{1}, \alpha=c_{1}$ hence, we have $c_{2} \tan (\theta)=c_{1}-\beta_{\mathrm{pl}}\left(c_{1}, \gamma, \mathcal{V}\right)$ and $c_{3} \cot (\phi)=\left(c_{1}-\gamma\right) \cos (\theta)$. In order to find $\gamma$ as a function of $\theta$, we replace $\beta=c_{1}-T_{2}^{l}$ and $\alpha=c_{1}$ in the formula of the volume (C.24) to obtain the following equation:

$$
\gamma^{3}-3 \ell_{23} T_{2}^{l} \gamma+\left(c_{1}-T_{2}^{l}\right)^{3}-c_{1}^{3}+3 c_{1} \ell_{23} T_{2}^{l}=0
$$


Using the property of symmetry $\Psi_{\mathrm{pl}}(\alpha, \beta, \gamma)=\Psi_{\mathrm{pl}}(\alpha, \gamma, \beta)$, the above formula writes $\Psi_{\mathrm{pl}}\left(c_{1}, c_{1}-T_{2}^{l}, \gamma\right)=$ 0 . As previously, we take the root $k=2$ to get $\gamma \leq \alpha-\beta$. Hence, after some simplifications, we obtain formula (16a) defined on $\left[\theta_{2}^{\mathrm{h}}, \theta_{3}\right]$. Note that we have used the relations $\beta_{\mathrm{pl}}\left(c_{1}, 0, \mathcal{V}\right)=f_{\mathcal{V}}^{23}\left(c_{1}\right)$ and $\beta_{\mathrm{pl}}\left(c_{1}, f_{\mathcal{V}}^{23}\left(c_{1}\right), \mathcal{V}\right)=0$ coming from (C.28).

For the limit curve $\Gamma_{\mathrm{h}}^{2}$ we chose the points $(0, \alpha, 0),\left(0, \beta, c_{3}\right)$ and $\left(c_{1}, \gamma, 0\right)$ to get $a_{1}=\frac{\alpha-\gamma}{c_{1} \alpha}, a_{2}=\frac{1}{\alpha}$, $a_{3}=\frac{\alpha-\beta}{c_{3} \alpha}$ and the limit is on $\left(c_{2}, \gamma\right)$ with $\gamma \in\left[0, f_{\mathcal{V}}^{13}\left(c_{2}\right)\right]$. We have $\gamma=c_{2}-T_{3}^{r}$ and $c_{3} \cot (\phi)=\left(c_{2}-\beta\right) \sin (\theta)$. In order to find $\beta$ as a function of $\theta$, we replace $\gamma=c_{2}-T_{3}^{r}$ and $\alpha=c_{2}$ in the formula of the volume (C.24) where $\ell_{23}$ is replaced $\ell_{13}$ to obtain the following equation:

$$
\beta^{3}-3 \ell_{13} T_{3}^{r} \beta+\left(c_{2}-T_{3}^{r}\right)^{3}-c_{2}^{3}+3 c_{2} \ell_{13} T_{3}^{r}=0
$$

Hence we obtain formula (16b) defined on $\left[\theta_{3}, \theta_{4}^{\mathrm{h}}\right]$.

For $\Gamma_{\mathrm{h}}^{3}$ we chose the points $(0,0, \alpha),\left(c_{1}, 0, \beta\right)$ and $\left(0, c_{2}, \gamma\right)$ to get $a_{1}=\frac{\alpha-\beta}{c_{1} \alpha}, a_{2}=\frac{\alpha-\gamma}{c_{2} \alpha}, a_{3}=\frac{1}{\alpha}$ and the limit is on $\left(c_{3}, \gamma\right)$ with $\left.\gamma \in\left[0, f_{\mathcal{V}}^{12}\left(c_{3}\right)\right]\right)$. We can prove that $T_{2}^{l}\left(c_{3}-\beta\right)=c_{1}\left(c_{3}-\gamma\right)$ and $\cot (\phi) \sec (\theta)=\frac{c_{1}}{c_{3}-\beta}$. In order to find $\beta$ as a function of $\theta$, we replace $\gamma=c_{3}+\frac{T_{2}^{l}}{c_{1}}\left(\beta-c_{3}\right)$ and $\alpha=c_{3}$ in the formula of the volume (C.24) where $\ell_{23}$ is replaced $\ell_{12}$ to obtain the following equation:

$$
\left(\frac{1}{c_{3}-\beta}-\frac{c_{1}+T_{2}^{l}}{c_{1} c_{3}}\right)^{3}+3 T_{2}^{l} \frac{\ell_{12}-2 c_{3}}{c_{1} c_{3}^{3}}\left(\frac{1}{c_{3}-\beta}-\frac{c_{1}+T_{2}^{l}}{c_{1} c_{3}}\right)+3 T_{2}^{l} \frac{\left(\ell_{12}-c_{3}\right)\left(c_{1}+T_{2}^{l}\right)}{c_{1}^{2} c_{3}^{4}}=0
$$

Hence using $c_{3}-\ell_{12}=\frac{c_{3}}{c_{1}}\left(c_{1}-\ell_{23}\right)$ we obtain equation (16c) defined on $\left[\theta_{2}^{\mathrm{h}}, \theta_{4}^{\mathrm{h}}\right]$.

Appendix C.6. Parametrization of the Hexa configuration

Appendix C.6.1. Parametrization in $(\alpha, \gamma)$

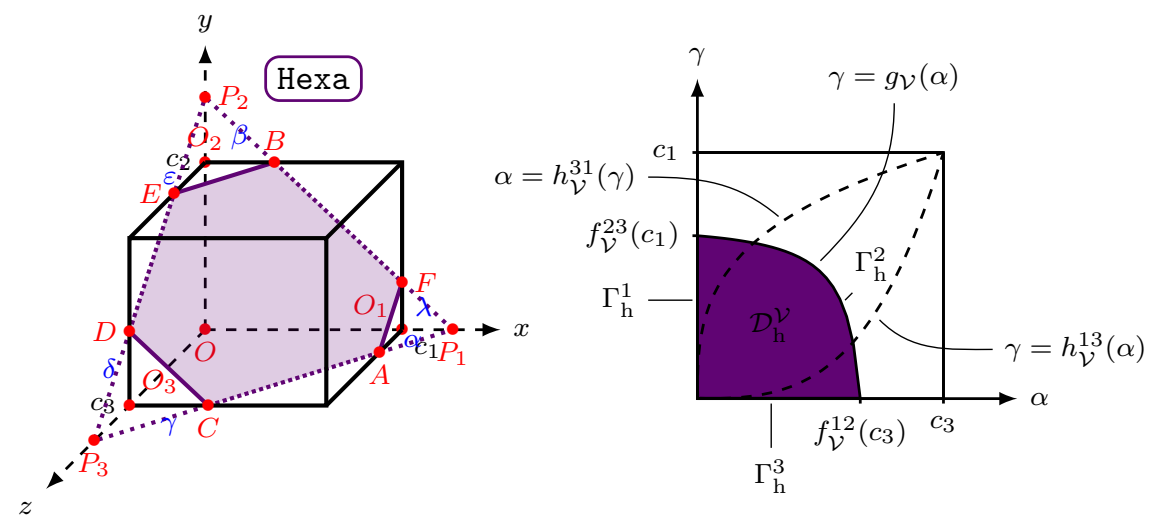

Figure C.16: Hexa configuration (left) and definition domain $\mathcal{D}_{h}^{\mathcal{V}}$ with $\chi>\frac{1}{6}$ (right).

In the Hexa configuration, the plane intersects the cell as presented in figure C.16. The coordinates of the intersection points are given by $A=\left(c_{1}, 0, \alpha\right), B=\left(\beta, c_{2}, 0\right), C=\left(\gamma, 0, c_{3}\right), D=\left(0, \delta, c_{3}\right), E=\left(0, c_{2}, \varepsilon\right)$, and $F=\left(c_{1}, \lambda, 0\right)$. The coefficients of the plane (C.2) are given by:

$$
a_{1}=\frac{c_{3}-\alpha}{c_{1} c_{3}-\alpha \gamma} \quad a_{2}=\frac{c_{3}\left(c_{1}-\beta\right)+\alpha(\beta-\gamma)}{c_{2}\left(c_{1} c_{3}-\alpha \gamma\right)} \quad a_{3}=\frac{c_{1}-\gamma}{c_{1} c_{3}-\alpha \gamma}
$$

Since the points $D, E$, and $F$ belongs to the plane, $\delta=\frac{c_{2} \gamma\left(c_{3}-\alpha\right)}{c_{3}\left(c_{1}-\beta\right)+\alpha(\beta-\gamma)}, \varepsilon=\frac{\beta\left(c_{3}-\alpha\right)}{c_{1}-\gamma}$, and $\lambda=\frac{c_{2} \alpha\left(c_{1}-\gamma\right)}{c_{3}\left(c_{1}-\beta\right)+\alpha(\beta-\gamma)}$.

We have $\beta, \gamma \in\left[0, c_{1}\right], \delta, \lambda \in\left[0, c_{2}\right]$ and $\alpha, \varepsilon \in\left[0, c_{3}\right]$. Therefore $0 \leq \frac{c_{2} \gamma\left(c_{3}-\alpha\right)}{c_{3}\left(c_{1}-\beta\right)+\alpha(\beta-\gamma)} \leq c_{2}$. The left inequality is satisfied if $c_{3} \beta+\alpha \gamma \leq c_{1} c_{3}+\alpha \beta$ and the right inequality is satisfied if $c_{3}(\beta+\gamma) \leq c_{1} c_{3}+\alpha \beta$. The 
second inequality implies the first one because $c_{3} \beta+\alpha \gamma \leq c_{3}(\beta+\gamma)$ and $\alpha \leq c_{3}$. We also have $0 \leq \frac{\beta\left(c_{3}-\alpha\right)}{c_{1}-\gamma} \leq c_{3}$. The left inequality is always satisfied and the right inequality is satisfied if $c_{3}(\beta+\gamma) \leq c_{1} c_{3}+\alpha \beta$. We also have $0 \leq \frac{c_{2} \alpha\left(c_{1}-\gamma\right)}{c_{3}\left(c_{1}-\beta\right)+\alpha(\beta-\gamma)} \leq c_{2}$. The left inequality is satisfied if $c_{3} \beta+\alpha \gamma \leq c_{1} c_{3}+\alpha \beta$ and the right inequality is always satisfied because $\left(c_{1}-\beta\right)\left(c_{3}-\alpha\right) \geq 0$. Therefore only one inequality stands:

$$
\beta \leq \frac{c_{3}\left(c_{1}-\gamma\right)}{c_{3}-\alpha}
$$

We introduce three points $P_{1}=\left(h_{1}, 0,0\right), P_{2}=\left(0, h_{2}, 0\right)$, and $P_{3}=\left(0,0, h_{3}\right)$ where $h_{1}=\frac{c_{1} c_{3}-\alpha \gamma}{c_{3}-\alpha}, h_{2}=$ $\frac{c_{2}\left(c_{1} c_{3}-\alpha \gamma\right)}{c_{3}\left(c_{1}-\beta\right)+\alpha(\beta-\gamma)}$, and $h_{3}=\frac{c_{1} c_{3}-\alpha \gamma}{c_{1}-\gamma}$. $P_{1}$ corresponds to the intersection of the planes $\{y=0\}$ and $\{z=0\}$ with the interface, $P_{2}$ corresponds to the intersection of the planes $\{x=0\}$ and $\{z=0\}$ with the interface and $P_{3}$ corresponds to the intersection of the planes $\{x=0\}$ and $\{y=0\}$ with the interface. The volume and the centroid of the domain $\mathrm{AFO}_{1} \mathrm{BEO}_{2} \mathrm{DCO}_{3} \mathrm{O}$ are computed using the additivity of the volume and the first momentum of the tetrahedrons $O P_{1} P_{2} P_{3}, O_{1} A F P_{1}, O_{2} B E P_{2}$, and $O_{3} C D P_{3}$. Therefore, the volume is given by:

$$
\mathcal{V}_{\mathrm{h}}(\alpha, \beta, \gamma)=\frac{c_{2}\left(c_{3}-\alpha\right)^{2}\left(c_{1}^{3}-\beta^{3}-\gamma^{3}\right)+3 c_{1} c_{2} \alpha\left(c_{1}-\gamma\right)\left(c_{1} c_{3}-\alpha \gamma\right)}{6\left(c_{1}-\gamma\right)\left(c_{3}\left(c_{1}-\beta\right)+\alpha(\beta-\gamma)\right)}
$$

The centroid is given by:

$$
\mathcal{C}_{h}(\alpha, \beta, \gamma)=\left[\begin{array}{c}
\frac{\left(c_{3}-\alpha\right)^{2}\left(c_{1}^{4}-\beta^{4}-\gamma^{4}\right)+2 c_{1}^{2} \alpha\left(c_{1}-\gamma\right)\left(2\left(c_{1} c_{3}-\alpha \gamma\right)+\alpha\left(c_{1}-\gamma\right)\right)}{4\left(\left(c_{3}-\alpha\right)^{2}\left(c_{1}^{3}-\beta^{3}-\gamma^{3}\right)+3 c_{1} \alpha\left(c_{1}-\gamma\right)\left(c_{1} c_{3}-\alpha \gamma\right)\right)} \\
\frac{c_{2}\left(\left(c_{3}-\alpha\right)^{3}\left(c_{1}^{4}-\beta^{4}-\gamma^{4}\right)+2 c_{1} \alpha\left(c_{1}-\gamma\right)\left(2\left(c_{1} c_{3}-\alpha \gamma\right)^{2}-c_{1} \alpha\left(c_{3}-\alpha\right)\left(c_{1}-\gamma\right)\right)-4 \beta^{3}\left(c_{3}-\alpha\right)^{2}\left(c_{3}\left(c_{1}-\beta\right)+\alpha(\beta-\gamma)\right)\right)}{4\left(c_{3}\left(c_{1}-\beta\right)+\alpha(\beta-\gamma)\right)\left(\left(c_{3}-\alpha\right)^{2}\left(c_{1}^{3}-\beta^{3}-\gamma^{3}\right)+3 c_{1} \alpha\left(c_{1}-\gamma\right)\left(c_{1} c_{3}-\alpha \gamma\right)\right)} \\
\frac{\left(c_{3}-\alpha\right)^{3}\left(c_{1}^{4}-\beta^{4}-\gamma^{4}\right)+2 c_{1} \alpha\left(c_{1}-\gamma\right)\left(2\left(c_{1} c_{3}-\alpha \gamma\right)^{2}-c_{1} \alpha\left(c_{3}-\alpha\right)\left(c_{1}-\gamma\right)\right)-4 \gamma^{3}\left(c_{3}-\alpha\right)^{2}\left(c_{3}\left(c_{1}-\gamma\right)\right)}{4\left(c_{1}-\gamma\right)\left(\left(c_{3}-\alpha\right)^{2}\left(c_{1}^{3}-\beta^{3}-\gamma^{3}\right)+3 c_{1} \alpha\left(c_{1}-\gamma\right)\left(c_{1} c_{3}-\alpha \gamma\right)\right)}
\end{array}\right]
$$

Note that the volume and the centroid are continuous between the Penta and the Hexa configurations since $\mathcal{V}_{\mathrm{h}}(0, \beta, \gamma)=\mathcal{V}_{\mathrm{p}}\left(c_{1}, \beta, \gamma\right)$ and $\mathcal{C}_{\mathrm{h}}(0, \beta, \gamma)=\mathcal{C}_{\mathrm{p}}\left(c_{1}, \beta, \gamma\right)$. By imposing a reference fixed volume $\mathcal{V}$ in $(\mathrm{C} .38), \beta$ is the solution of the following third degree polynomial:

$$
\Psi_{\mathrm{h}}(\alpha, \beta, \gamma)=\beta^{3}-\frac{6 \mathcal{V}\left(c_{1}-\gamma\right)}{c_{2}\left(c_{3}-\alpha\right)} \beta-\frac{\left(c_{1}-\gamma\right)}{c_{2}\left(c_{3}-\alpha\right)^{2}}\left(c_{2}\left(c_{3}-\alpha\right)^{2}\left(c_{1}^{2}+c_{1} \gamma+\gamma^{2}\right)+3\left(c_{1} c_{3}-\alpha \gamma\right)\left(c_{1} c_{2} \alpha-2 \mathcal{V}\right)\right)=0
$$

Let us find the definition domain of the Hexa configuration:

$$
\mathcal{D}_{h}^{\mathcal{V}}=\left\{(\alpha, \gamma) \in\left[0, c_{3}\right] \times\left[0, c_{1}\right] \mid 0 \leq \beta_{h}(\alpha, \gamma, \mathcal{V}) \leq c_{1} \text { and } \beta_{h}(\alpha, \gamma, \mathcal{V}) \leq \frac{c_{3}\left(c_{1}-\gamma\right)}{c_{3}-\alpha}\right\}
$$

where $\beta_{h}(\alpha, \gamma, \mathcal{V})$ is solution of equation (C.40). To find this domain, let use first find its boundaries. From equation (C.40), we have:

$$
\begin{array}{ll}
\text { For } \beta=0 & \Psi_{\mathrm{h}}(\alpha, 0, \gamma)=-\left(c_{1}-\gamma\right)\left(\gamma-g_{\mathcal{V}}(\alpha)\right)\left(\gamma-\tilde{g}_{\mathcal{V}}(\alpha)\right)=0 \\
\text { For } \beta=\frac{c_{3}\left(c_{1}-\gamma\right)}{c_{3}-\alpha} & \Psi_{\mathrm{h}}\left(\alpha, \frac{c_{3}\left(c_{1}-\gamma\right)}{c_{3}-\alpha}, \gamma\right)=\frac{\alpha\left(c_{1}-\gamma\right)\left(\alpha^{2}+3 c_{3}\left(c_{3}-\alpha\right)\right)}{\left(c_{3}-\alpha\right)^{3}}\left(\gamma-h_{\mathcal{V}}^{13}(\alpha)\right)\left(\gamma-\tilde{h}_{\mathcal{V}}^{13}(\alpha)\right)=0 \\
\text { For } \beta=c_{1} & \Psi_{\mathrm{h}}\left(\alpha, c_{1}, \gamma\right)=\frac{\gamma\left(\gamma^{2}+3 c_{1}\left(c_{1}-\gamma\right)\right)}{\left(c_{3}-\alpha\right)^{2}}\left(\alpha-h_{\mathcal{V}}^{31}(\gamma)\right)\left(\alpha-\tilde{h}_{\mathcal{V}}^{31}(\gamma)\right)=0
\end{array}
$$

where $\tilde{g}_{\mathcal{V}}$ and $\tilde{h}_{\mathcal{V}}^{13}$ are the conjugate roots of $g_{\mathcal{V}}$ and $h_{\mathcal{V}}$ defined in equation (C.7) and (C.8). These functions are well defined, but outside of the domain $\left[0, c_{3}\right] \times\left[0, c_{1}\right]$. Furthermore, $h_{\mathcal{V}}^{31}$ is the same function as $h_{\mathcal{V}}^{13}$ where $c_{1}$ and $c_{3}$ are swapped.

The possible limits of the domain $\mathcal{D}_{h}^{\mathcal{V}}$ are necessary in the following list: $\alpha=0, \alpha=c_{3}, \gamma=0, \gamma=c_{1}$, $\gamma=g_{\mathcal{V}}(\alpha), \gamma=h_{\mathcal{V}}^{13}(\alpha)$, or $\alpha=h_{\mathcal{V}}^{31}(\gamma)$. These curves form a partition of the domain $\left[0, c_{3}\right] \times\left[0, c_{1}\right]$ in six regions such as presented in figure C.16. Thus $\mathcal{D}_{h}^{\mathcal{V}}$ is the reunion of some - possibly only one - of these 
regions. It is easy to check numerically by picking one value in these regions that three of these domains can not correspond since $\beta \notin \mathbb{R}, \beta \notin\left[0, c_{1}\right]$ or $\beta>\frac{c_{3}\left(c_{1}-\gamma\right)}{c_{3}-\alpha}$ in these domains. Therefore, the definition domain of the Hexa is necessarily given by:

$$
\mathcal{D}_{h}^{\mathcal{V}}=\left\{(\alpha, \gamma) \in\left[0, c_{3}\right] \times\left[0, c_{1}\right] \mid \gamma \leq g_{\mathcal{V}}(\alpha)\right\}
$$

The limit curves are given by $\Gamma_{\mathrm{h}}^{1}: \gamma \mapsto(0, \gamma)$ where $\gamma \in\left[0, f_{\mathcal{V}}^{23}\left(c_{1}\right)\right], \Gamma_{\mathrm{h}}^{2}: \alpha \mapsto\left(\alpha, g_{\mathcal{V}}(\alpha)\right)$ where $\alpha \in\left[0, f_{\mathcal{V}}^{12}\left(c_{3}\right)\right]$ and $\Gamma_{\mathrm{h}}^{3}: \alpha \mapsto(\alpha, 0)$ where $\alpha \in\left[0, f_{\mathcal{V}}^{12}\left(c_{3}\right)\right]$.

We can show that the discriminant of the third degree polynomial (C.40) is negative on $\mathcal{D}_{\mathrm{h}}^{\mathcal{V}}$ and the only root that verifies $0 \leq \beta \leq c_{1}$ and $\beta \leq \frac{c_{3}\left(c_{1}-\gamma\right)}{c_{3}-\alpha}$ is given by:

$$
\beta_{\mathrm{h}}(\alpha, \gamma, \mathcal{V})=2 \sqrt{\frac{2 \mathcal{V}\left(c_{1}-\gamma\right)}{c_{2}\left(c_{3}-\alpha\right)}} \cos \left(\frac{1}{3} \arccos \left(\frac{c_{2}\left(c_{3}-\alpha\right)^{2}\left(c_{1}^{2}+c_{1} \gamma+\gamma^{2}\right)+3\left(c_{1} c_{3}-\alpha \gamma\right)\left(c_{1} c_{2} \alpha-2 \mathcal{V}\right)}{4 \mathcal{V} \sqrt{2 \mathcal{V}\left(c_{1}-\gamma\right)\left(c_{3}-\alpha\right) / c_{2}}}\right)+\frac{4 \pi}{3}\right)
$$

The proof of this formula follows the same line as for the PentaL configuration. The discriminant $\Delta_{\mathrm{h}}$ can be seen as a third degree polynomial in the volume $\mathcal{V}$ instead of a fourth degree polynomial in $\alpha$ or $\gamma$ using a suitable change of the quantifiers. Then, we compute the roots of the derivative of $\Delta_{\mathrm{h}}$ and show appropriate inequalities that prove the desired result. Afterwards, we use the proposition 1 with $\mathcal{K}=c_{1}$ and $\mathcal{K}=\frac{c_{3}\left(c_{1}-\gamma\right)}{c_{3}-\alpha}$ to prove that only the root $k=2$ verifies $0 \leq \beta \leq c_{1}$ and $\beta \leq \frac{c_{3}\left(c_{1}-\gamma\right)}{c_{3}-\alpha}$. Nevertheless, the proof required the computation and the comparison of the curves $\left\{Y_{\mathrm{h}}=\frac{1}{2}\right\}$ and $\left\{\Psi_{\mathrm{h}}=0\right\}$ because $\Psi_{\mathrm{h}}$ is not of constant sign on $\mathcal{D}_{\mathrm{h}}^{\mathcal{V}}$ like in the PentaL case.

Appendix C.6.2. Parametrization in $(\theta, \phi)$ of the centroid and the limit curves in $(\theta, \phi)$

From equations (C.3) and (C.37), we get $\tan (\theta)=\frac{c_{3}\left(c_{1}-\beta\right)+\alpha(\beta-\gamma)}{c_{2}\left(c_{3}-\alpha\right)}$ and $\cot (\phi)=\frac{c_{1}-\gamma}{c_{3}-\alpha} \cos (\theta)$. These equations are difficult to solve directly because of the expression of $\beta$ in (C.41). Instead, the trick is to express $\alpha$ and $\gamma$ with respect to $\beta, \theta$, and $\phi$ and to get a third degree polynomial on $\beta$ from the equation of the volume (C.38). We introduce the following notations:

$$
\alpha=c_{3} \frac{\beta-c_{1}+T_{2}^{l}}{T_{3}^{l}} \quad \gamma=\beta+T_{2}^{l}-T_{3}^{l} \quad T_{2}^{l}=c_{2} \tan (\theta) \quad T_{3}^{l}=c_{3} \cot (\phi) \sec (\theta)
$$

We replace these expressions in (C.38) to obtain the following third degree polynomial in $\beta$ :

$$
\left(\beta-\frac{1}{2}\left(c_{1}-T_{2}^{l}+T_{3}^{l}\right)\right)^{3}-\frac{3}{4} T_{4}^{l}\left(\beta-\frac{1}{2}\left(c_{1}-T_{2}^{l}+T_{3}^{l}\right)\right)-\frac{3}{2} T_{2}^{l} T_{3}^{l}\left(c_{1}-\ell_{23}\right)=0
$$

where $T_{4}^{l}=4 c_{1} T_{3}^{l}-\left(c_{1}-T_{2}^{l}+T_{3}^{l}\right)^{2}$. We already know that the three roots are real. Like for the Penta configuration, we can apply the extended proposition with $\mathcal{K}=0, \mathcal{K}=c_{1}$, and $\mathcal{K}=\frac{c_{3}\left(c_{1}-\gamma\right)}{c_{3}-\alpha}$ to $\alpha, \beta$ and $\gamma$ and show that the only root that verifies $\beta \leq \frac{c_{3}\left(c_{1}-\gamma\right)}{c_{3}-\alpha}, 0 \leq \alpha \leq c_{1}, 0 \leq \beta \leq c_{1}$, and $0 \leq \gamma \leq c_{1}$ is given by the root $k=2$ :

$$
\beta_{\mathrm{h}}(\theta, \phi, \mathcal{V})=\frac{1}{2}\left(c_{1}-T_{2}^{l}+T_{3}^{l}\right)+\sqrt{T_{4}^{l}} \mathcal{X}_{\mathrm{h}} \quad \mathcal{X}_{\mathrm{h}}=\cos \left(\frac{1}{3} \arccos \left(\frac{6 T_{2}^{l} T_{3}^{l}\left(c_{1}-\ell_{23}\right)}{\left(T_{4}^{l}\right)^{\frac{3}{2}}}\right)+\frac{4 \pi}{3}\right)
$$

where $c_{1} \geq \ell_{23}$ and where $\mathcal{X}_{\mathrm{h}}$ verifies the equation:

$$
\left(\mathcal{X}_{\mathrm{h}}\right)^{3}-\frac{3}{4} \mathcal{X}_{\mathrm{h}}-\frac{3 T_{2}^{l} T_{3}^{l}\left(c_{1}-\ell_{23}\right)}{2\left(T_{4}^{l}\right)^{\frac{3}{2}}}=0
$$

To obtain the final parametrization (22), we replace $\alpha, \beta$ and $\gamma$ in (C.39) by their expressions given in (C.42) and (C.43). We obtain a fourth degree polynomial in $\mathcal{X}_{\mathrm{h}}$ for each component. Then we use equation (C.44) 
to get a second degree polynomial in each component. Finally, after some simplifications, we obtain equation (22).

To compute the partial derivatives of the centroid using the formula of Chen \& Zhang [19], use the coordinates of the vertices of the interface given in order by $A=\left(c_{1}, 0, \alpha_{\mathrm{h}}\right), F=\left(c_{1}, \lambda_{\mathrm{h}}, 0\right), B=\left(\beta_{\mathrm{h}}, c_{2}, 0\right)$, $E=\left(0, c_{2}, \varepsilon_{\mathrm{h}}\right), D=\left(0, \delta_{\mathrm{h}}, c_{3}\right)$, and $C=\left(\gamma_{\mathrm{h}}, 0, c_{3}\right)$ using (C.43) and the relations:

$$
\gamma_{\mathrm{h}}=\beta_{\mathrm{h}}+T_{2}^{l}-T_{3}^{l} \quad \alpha_{\mathrm{h}}=c_{3} \frac{\beta_{\mathrm{h}}-c_{1}+T_{2}^{l}}{T_{3}^{l}} \quad \delta_{\mathrm{h}}=\frac{c_{2} \gamma_{\mathrm{h}}}{T_{2}^{l}} \quad \varepsilon_{\mathrm{h}}=\frac{c_{3} \beta_{\mathrm{h}}}{T_{3}^{l}} \quad \lambda_{\mathrm{h}}=c_{2} \frac{\beta_{\mathrm{h}}+T_{2}^{l}-c_{1}}{T_{2}^{l}}
$$

\section{Appendix C.7. Technical lemmas}

\section{Appendix C.7.1. General inequality}

Proposition 1. Let $\Psi(\beta)=\beta^{3}+p \beta+q$ a third degree polynomial in $\beta$. If the discriminant $\Delta=4 p^{3}+27 q^{2} \leq 0$, which implies $p \leq 0$, the roots of $\Psi(\beta)$ are real and given by:

$$
\forall k \in \llbracket 0,2 \rrbracket \quad \beta_{k}=2 \sqrt{-\frac{p}{3}} \cos \left(\frac{1}{3} \arccos \left(\frac{-q}{2 \sqrt{\left(-\frac{p}{3}\right)^{3}}}\right)+\frac{2 k \pi}{3}\right)
$$

Then, the sign of $\beta-\mathcal{K}$ is the same as $\cos \left(\frac{1}{3} \arccos (X)+\frac{2 k \pi}{3}\right)-Y$ where $X=\frac{-q}{2 \sqrt{(-p / 3)^{3}}}$ and $Y=\frac{\mathcal{K}}{2 \sqrt{-p / 3}}$. Furthermore, suppose that $-1 \leq X \leq 0$. Then, the three roots verify $\beta_{1} \leq 0, \beta_{0}, \beta_{2} \geq 0$ and we have the

\begin{tabular}{|c|c|c|c|}
\hline$Y \leq 0$ & $0 \leq Y \leq \frac{1}{2}$ & $\frac{1}{2} \leq Y \leq 1$ & $Y \geq 1$ \\
\hline$\beta_{0} \geq \mathcal{K}$ & $\beta_{0} \geq \mathcal{K}$ & $\begin{array}{ll}\text { if } \Psi \leq 0 & \beta_{0} \geq \mathcal{K} \\
\text { if } \Psi \geq 0 & \beta_{0} \leq \mathcal{K}\end{array}$ & $\beta_{0} \leq \mathcal{K}$ \\
\hline$\beta_{2} \geq \mathcal{K}$ & $\begin{array}{ll}\text { if } \Psi \leq 0 & \beta_{2} \leq \mathcal{K} \\
\text { if } \Psi \geq 0 & \beta_{2} \geq \mathcal{K}\end{array}$ & $\beta_{2} \leq \mathcal{K}$ & $\beta_{2} \leq \mathcal{K}$ \\
\hline
\end{tabular}
following inequalities where $\Psi$ stands for $\Psi(\mathcal{K})$ :

Proof. We have the following identities for $x \in[0,2 \pi]$ :

$$
\cos (\arccos (x))=x \quad \arccos (\cos (x))= \begin{cases}x & \text { if } \quad 0 \leq x \leq \pi \\ 2 \pi-x & \text { if } \quad \pi<x<2 \pi\end{cases}
$$

It is easy to check that:

$$
X-\cos (3 \arccos (Y))=X-\left(4 Y^{3}-3 Y\right)=-\frac{\Psi(\mathcal{K})}{2 \sqrt{(-p / 3)^{3}}}
$$

We have the inequalities $(4 k+1) \frac{\pi}{6} \leq \frac{1}{3}(\arccos (X)+2 k \pi) \leq(4 k+2) \frac{\pi}{6}$, so $\beta_{1} \leq 0$ and $\beta_{0}, \beta_{2} \geq 0$. In the first part of the proof, we use only the hypothesis $-1 \leq X \leq 0$ and not the expression of $X$ and $Y$. If $0 \leq Y \leq \frac{1}{2}$, we have $\frac{\pi}{6} \leq \frac{1}{3}(\arccos (X)) \leq \frac{\pi}{3} \leq \arccos (Y) \leq \frac{\pi}{2}$. Since the cosine function is monotonically decreasing on $\left[\frac{\pi}{6}\right.$, $\left.\frac{\pi}{2}\right]$, we get by composition $\beta_{0} \geq \mathcal{K}$. If $\frac{1}{2} \leq Y \leq 1$, we have $-\frac{\pi}{2} \leq \frac{1}{3}(\arccos (X)-2 \pi) \leq-\frac{\pi}{3} \leq-\arccos (Y) \leq 0$. Since the cosine function is monotonically increasing on $\left[-\frac{\pi}{2}, 0\right]$ we get by composition $\beta_{2} \leq \mathcal{K}$. In the second part of the proof, we will use the identity (C.47). First, we suppose that $\Psi \leq 0$. Since arccos is monotonically decreasing, using (C.47), we get $\arccos (X) \leq \arccos (\cos (3 \arccos (Y)))$. If $\frac{1}{2} \leq Y \leq 1$, from (C.46), we get $\arccos (X) \leq 3 \arccos (Y)$, so $\frac{\pi}{6} \leq \frac{1}{3} \arccos (X) \leq \arccos (Y) \leq \frac{\pi}{3}$. Since the cosine function is monotonically decreasing on $\left[\frac{\pi}{6}, \frac{\pi}{3}\right]$, we get $\beta_{0} \geq \mathcal{K}$. If $0 \leq Y \leq 1 / 2$, from (C.46), we get $\arccos (X) \leq 2 \pi-3 \arccos (Y)$, so $\frac{\pi}{3} \leq \arccos (Y) \leq \frac{1}{3}(2 \pi-\arccos (X)) \leq \frac{\pi}{2}$. Since the cosine function is monotonically decreasing on $\left[\frac{\pi}{3}, \frac{\pi}{2}\right]$, we get $\beta_{2} \leq \mathcal{K}$. If $\Psi \geq 0$, the proof is the same but the inequalities are reversed because of (C.47). 
Appendix C.7.2. Analytical expression for the PentaL configuration of $\beta_{\mathrm{pl}}$ on $\mathcal{D}_{\mathrm{pl}}^{\mathcal{V}}$

We want to find an analytical expression of $\beta$ that verifies $0 \leq \beta \leq \alpha-\gamma$ on $\mathcal{D}_{\mathrm{pl}}^{\mathcal{V}}$. The discriminant of the third degree polynomial (C.24) is given by $\Delta=27(\alpha-\gamma)^{2} \Delta_{p}$ where:

$$
\Delta_{p}\left(\alpha, \gamma, \ell_{23}\right)=\left(\alpha^{2}+\alpha \gamma+\gamma^{2}-3 \ell_{23} \alpha\right)^{2}-4(\alpha-\gamma)\left(\ell_{23}\right)^{3}
$$

First, let us show that $\Delta_{p}$ is negative on the definition domain of the Penta $\mathcal{D}_{\mathrm{pl}}^{\mathcal{V}}$ which writes:

$$
\forall c_{1}>0, \quad \forall \ell_{23} \in\left[0, c_{1}\right], \quad \forall \alpha \in\left[\ell_{23}, \min \left(c_{1}, 3 \ell_{23}\right)\right], \quad \forall \gamma \in\left[0, f_{\mathcal{V}}^{23}(\alpha)\right], \quad \Delta_{p}\left(\alpha, \gamma, \ell_{23}\right) \leq 0
$$

This expression is a fourth degree polynomial in $\alpha$ and $\gamma$. To prove that $\Delta_{p}$ is negative appears to be a complicated task. Instead we can see $\Delta_{p}$ as a third degree polynomial in $\ell_{23}$. As illustrated in figure C.17, it is equivalent to show that:

$$
\forall c_{1}>0, \quad \forall \alpha \in\left[0, c_{1}\right], \quad \forall \gamma \in[0, \alpha], \quad \forall \ell_{23} \in\left[\ell_{23}^{\min }(\alpha, \gamma), \ell_{23}^{\max }(\alpha, \gamma)\right], \quad \Delta_{p}\left(\alpha, \gamma, \ell_{23}\right) \leq 0
$$

where $\ell_{23}^{\min }(\alpha, \gamma)=\frac{\alpha^{2}+\alpha \gamma+\gamma^{2}}{3 \alpha}$ and $\ell_{23}^{\max }(\alpha, \gamma)=\alpha$.
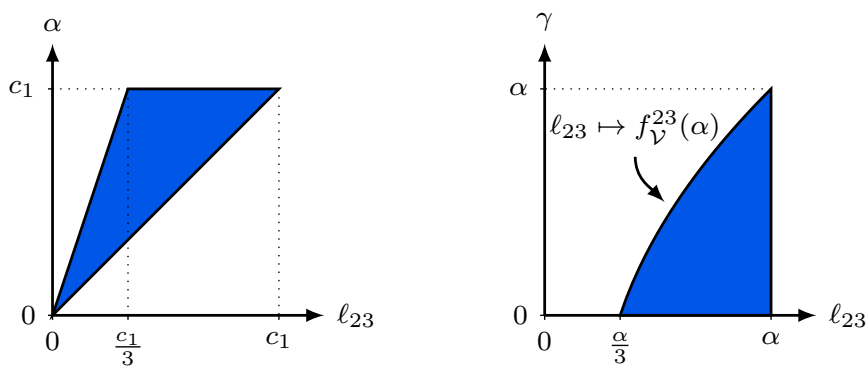

Figure C.17: Illustration of the inversion of the quantifiers.

The partial derivative of $\Delta_{p}$ with respect to $\ell_{23}$ writes:

$$
\frac{\partial \Delta_{p}}{\partial \ell_{23}}=-6\left(2(\alpha-\gamma)\left(\ell_{23}\right)^{2}-3 \alpha^{2} \ell_{23}+\alpha\left(\alpha^{2}+\alpha \gamma+\gamma^{2}\right)\right)
$$

The roots of second degree polynomial $\frac{\partial \Delta_{p}}{\partial \ell_{23}}$ are given by $\ell_{23}^{ \pm}(\alpha, \gamma)=\frac{3 \alpha^{2} \pm \sqrt{\alpha^{4}+8 \alpha \gamma^{3}}}{4(\alpha-\gamma)}$. We have the following inequalities:

$$
\ell_{23}^{\min }(\alpha, \gamma) \leq \ell_{23}^{-}(\alpha, \gamma) \leq \ell_{23}^{\max }(\alpha, \gamma) \leq \ell_{23}^{+}(\alpha, \gamma)
$$

The right and the middle inequalities are equivalent to $8 \alpha \gamma(\alpha-\gamma)^{2} \geq 0$. The left one is equivalent to $16\left(\alpha^{3}-\gamma^{3}\right)^{2} \geq 0$. We also have:

$$
\Delta_{p}\left(\alpha, \gamma, \ell_{23}^{\min }(\alpha, \gamma)\right)=-4(\alpha-\gamma)\left(\ell_{23}^{\min }(\alpha, \gamma)\right)^{3} \leq 0 \quad \Delta_{p}\left(\alpha, \gamma, \ell_{23}^{\max }(\alpha, \gamma)\right)=-(\alpha-\gamma)(3 \alpha+\gamma) \gamma^{2} \leq 0
$$

The second degree polynomial $\frac{\partial \Delta_{p}}{\partial \ell_{23}}$ is positive on the interval $\left[\ell_{23}^{-}, \ell_{23}^{+}\right]$and negative outside. Hence $\ell_{23} \mapsto$ $\Delta_{p}\left(\alpha, \gamma, \ell_{23}\right)$ is monotonically increasing on $\left[\ell_{23}^{-}, \ell_{23}^{+}\right]$and monotonically decreasing elsewhere. Therefore, together with (C.50) and the inequalities (C.49), we get equation (C.48). The three solutions of (C.24) are then real and given by:

$$
\tilde{\beta}_{k}=2 \sqrt{\ell_{23}(\alpha-\gamma)} \cos \left(\frac{1}{3} \arccos \left(X_{\mathrm{pl}}\right)+\frac{2 k \pi}{3}\right) \quad X_{\mathrm{pl}}=\frac{\alpha^{2}+\alpha \gamma+\gamma^{2}-3 \ell_{23} \alpha}{2 \ell_{23} \sqrt{\ell_{23}(\alpha-\gamma)}}
$$


where $k \in\{0,1,2\}$. Now, let us show that the root $k=2$ is the only one which verifies $0 \leq \tilde{\beta}_{2} \leq \alpha-\gamma$. We introduce $\mathcal{K}=\alpha-\gamma$ and the notations:

$$
Y_{\mathrm{pl}}=\frac{\alpha-\gamma}{2 \sqrt{\ell_{23}(\alpha-\gamma)}}=\sqrt{\frac{\alpha-\gamma}{4 \ell_{23}}}
$$

On $\mathcal{D}_{\mathrm{pl}}^{\mathcal{V}}$, we have $-1 \leq X_{\mathrm{pl}} \leq 0-$ since $\Delta_{p} \leq 0-$ and $0 \leq Y_{\mathrm{pl}} \leq \frac{\sqrt{3}}{2} \leq 1$ since $\gamma \geq \alpha-3 \ell_{23}$. We also have:

$$
\Psi_{\mathrm{pl}}(\alpha, \alpha-\gamma, \gamma)=3 \gamma(\alpha-\gamma)\left(\ell_{23}-\alpha\right) \leq 0
$$

Using the proposition 1 , we get $\tilde{\beta}_{0} \geq \alpha-\gamma, \tilde{\beta}_{1} \leq 0$, and $\tilde{\beta}_{2} \leq \alpha-\gamma$. Therefore, we obtain the formula of the PentaL configuration (C.27).

\section{References}

[1] F. Gibou, R. Fedkiw, S. Osher, A review of level-set methods and some recent applications, Journal of Computational Physics 353 (2018) 82-109 (2018).

[2] G. Tryggvason, B. Bunner, A. Esmaeeli, D. Juric, N. Al-Rawahi, W. Tauber, J. Han, S. Nas, Y.-J. Jan, A front-tracking method for the computations of multiphase flow, Journal of computational physics 169 (2) (2001) $708-759$ (2001).

[3] V. Dyadechko, M. Shashkov, Moment-of-fluid interface reconstruction, Los Alamos National Laboratory Report LA-UR-05$7571(2005)$.

[4] V. Dyadechko, M. Shashkov, Moment-of-fluid interface reconstruction, Los Alamos National Laboratory Report LA-UR-071537 (2007).

[5] H. T. Ahn, M. Shashkov, Multi-material interface reconstruction on generalized polyhedral meshes, Journal of Computational Physics 226 (2) (2007) 2096-2132 (2007).

[6] V. Dyadechko, M. Shashkov, Reconstruction of multi-material interfaces from moment data, Journal of Computational Physics 227 (11) (2008) 5361-5384 (2008).

[7] H. T. Ahn, M. Shashkov, Adaptive moment-of-fluid method, Journal of Computational Physics 228 (8) (2009) 2792-2821 (2009).

[8] H. Anbarlooei, K. Mazaheri, 'moment of fluid'interface reconstruction method in axisymmetric coordinates, International Journal for Numerical Methods in Biomedical Engineering 27 (10) (2011) 1640-1651 (2011).

[9] S. Galera, J. Breil, P.-H. Maire, A 2d unstructured multi-material cell-centered arbitrary lagrangian-eulerian (ccale) scheme using mof interface reconstruction, Computers \& Fluids 46 (1) (2011) 237-244 (2011).

[10] S. P. Schofield, M. A. Christon, Effects of element order and interface reconstruction in fem/volume-of-fluid incompressible flow simulation, International Journal for Numerical Methods in Fluids 68 (11) (2012) 1422-1437 (2012).

[11] J. Breil, T. Harribey, P.-H. Maire, M. Shashkov, A multi-material reale method with mof interface reconstruction, Computers \& Fluids 83 (2013) 115-125 (2013).

[12] M. Jemison, E. Loch, M. Sussman, M. Shashkov, M. Arienti, M. Ohta, Y. Wang, A coupled level set-moment of fluid method for incompressible two-phase flows, Journal of Scientific Computing 54 (2-3) (2013) 454-491 (2013).

[13] R. N. Hill, M. Shashkov, The symmetric moment-of-fluid interface reconstruction algorithm, Journal of Computational Physics 249 (2013) 180-184 (2013).

[14] M. B. Friess, J. Breil, P.-H. Maire, M. Shashkov, A multi-material ccale-mof approach in cylindrical geometry, Communications in Computational Physics 15 (2) (2014) 330-364 (2014).

[15] M. Jemison, M. Sussman, M. Arienti, Compressible, multiphase semi-implicit method with moment of fluid interface representation, Journal of Computational Physics 279 (2014) 182-217 (2014).

[16] M. Jemison, M. Sussman, M. Shashkov, Filament capturing with the multimaterial moment-of-fluid method, Journal of Computational Physics 285 (2015) 149-172 (2015).

[17] X. Chen, X. Zhang, An improved 3d mof method based on analytical partial derivatives, Journal of Computational Physics 326 (2016) 156-170 (2016).

[18] A. Lemoine, S. Glockner, J. Breil, Moment-of-fluid analytic reconstruction on 2d cartesian grids, Journal of Computational Physics 328 (2017) 131-139 (2017).

[19] X. Chen, X. Zhang, An improved 2d mof method by using high order derivatives, Journal of Computational Physics 349 (2017) 176-190 (2017).

[20] E. Kikinzon, M. Shashkov, R. Garimella, Establishing mesh topology in multi-material cells: enabling technology for robust and accurate multi-material simulations, Computers \& Fluids (2018).

[21] A. Asuri Mukundan, T. Ménard, A. Berlemont, J. C. B. de Motta, Interface reconstruction method for multiphase flows in under-resolved regions, in: International Conference on Computational Fluid Dynamics, 2018, pp. 1-17 (Jul 2018). URL http://www.iccfd.org/iccfd10/papers/ICCFD10-013-Paper.pdf

[22] F. Qing, X. Yu, Z. Jia, A robust mof method applicable to severely deformed polygonal mesh, Journal of Computational Physics 377 (2019) 162-182 (2019).

[23] W. J. Rider, D. B. Kothe, Reconstructing volume tracking, Journal of computational physics 141 (2) (1998) $112-152$ (1998). 
[24] S. Diot, M. M. François, An interface reconstruction method based on an analytical formula for 3d arbitrary convex cells, Journal of Computational Physics 305 (2016) 63-74 (2016).

[25] R. Nürnberg, Calculating the area and centroid of a polygon in $2 \mathrm{~d}$ (2013).

URL http://www.ma.ic.ac.uk/ rn/centroid.pdf

[26] Notus website, https://notus-cfd.org (2019).

[27] M. Coquerelle, S. Glockner, A fourth-order accurate curvature computation in a level set framework for two-phase flows subjected to surface tension forces, Journal of Computational Physics 305 (2016) 838-876 (2016).

[28] J. Picot, S. Glockner, Reduction of the discretization stencil of direct forcing immersed boundary methods on rectangular cells: The ghost node shifting method, Journal of Computational Physics 364 (2018) 18 - 48 (2018). doi:https: //doi.org/10.1016/j.jcp.2018.02.047.

URL http://www.sciencedirect.com/science/article/pii/S0021999118301360

[29] R. Swinbank, R. J. Purser, Fibonacci grids: A novel approach to global modelling, Quarterly Journal of the Royal Meteorological Society 132 (619) (2006) 1769-1793 (2006).

[30] R. Fletcher, Practical methods of optimization, John Wiley \& Sons, 1987 (1987). 